AFFECTIVE VIDEO ON THE WEB

EXPLORING PRESENTATION AND TASK EFFECTS

Ria Verleur 
Thesis, University of Twente

ISBN 978-90-365-2706-4

(C) 2008 Ria Verleur

Cover Design: Johan Jonker \& Ria Verleur

Printed by PrintPartners Ipskamp, Enschede 


\section{AFFECTIVE VIDEO ON THE WEB \\ EXPLORING PRESENTATION AND TASK EFFECTS}

\section{PROEFSCHRIFT}

ter verkrijging van

de graad van doctor aan de Universiteit Twente, op gezag van de rector magnificus, prof. dr. W.H.M. Zijm, volgens besluit van het College voor Promoties in het openbaar te verdedigen op vrijdag 17 oktober 2008 om 15.00 uur

door

Ria Verleur

geboren op 25 mei 1966

te Utrecht 
Dit proefschrift is goedgekeurd door

de promotor: prof. dr. ir. P. W. Verhagen

de assistent-promotor: dr. A. Heuvelman 
We shall not cease from exploration And the end of all our exploring Will be to arrive where we started And know the place for the first time.

T.S. Eliot, "Little Gidding" 
Promotion Committee

Prof. dr. Jules M. Pieters, University of Twente, Chair and Secretary

Prof. dr. ir. Pløn W. Verhagen, University of Twente, Promotor

Dr. Ard Heuvelman, University of Twente, Assistant Promotor

Prof. dr. Johannes W.J. Beentjes, Radboud Universiteit Nijmegen

Prof. dr. Jan A.G.M. van Dijk, University of Twente

Prof. dr. Ingrid Heynderickx, Technische Universiteit Delft

Prof. dr. Allison H. Littlejohn, Glasgow Caledonian University

Prof. dr. Jef C.M.M. Moonen, University of Twente 
To Eric, Lisa, Marleen, and Jeroen 



\section{Contents}

$\begin{array}{lll}\text { Chapter } 1 & \text { Introduction } & 1\end{array}$

Affective Video and Educational Tasks on the Web

Chapter 2 Theoretical Framework

9

Context for Studying Presentation and Task Effects of

Affective Video on the Web

Chapter 3 Web Video Display

Affective Responses to Video: Do Medium and

Image Size Matter?

Chapter 4 Web Video Context

Can Mood-inducing Videos Affect Unrelated (Problem-

solving) Activities in a Web-based Environment?

Chapter 5 Web Video Design

65

What is the Impact of Web Trigger Video Design

on Emotional Responses and Related Task Activities?

Chapter 6 General Discussion

Presentation and Task Effects of Affective Video on the Web

References

Appendices

Samenvatting (Summary in Dutch)

Dankwoord (Acknowledgements in Dutch) 



\section{Introduction}

\section{Affective Video and Educational Tasks on the Web}

Developments in computer and internet technology have led to an emerged interest in and variety of applications of video in web-based learning environments. In this introductory chapter we present a brief overview of video properties that may be exploited for the effectiveness of these applications with a focus on the affect-evoking potential of video. The related body of knowledge is generally based on traditional settings of video uses. The purpose of the present PhD study is to explore whether and, if so, how video applied in a web environment can be effective in evoking affective responses and whether these responses may influence task performance.

\subsection{Affective Video in Everyday Life}

Stephens argued in 1998 that we were in a transition from a culture dominated by the printed word to one dominated by moving images (Stephens, 1998). Almost a decade later, this transformation seems to be a fact. The omnipresence of moving images of all sizes in all kinds of equipment has led to a continuously recurring confrontation with audiovisual messages that increasingly take the form of video. Video is entering our lives through television, cinema, mobile phones, iPods, game consoles, and computer applications with a prominent place for the Internet. The heavy use of video sites such as YouTube, which started only a few years ago in 2005, speaks for itself as does the almost routine use of video clips on all kinds of Web sites. At the same time the content and form of messages has changed. Images, also in news broadcasts, have become more affective. They contain more emotional content or are presented more dramatically than a few years ago (Grabe, Zhou, \& Barnett, 2001; Grabe, Zhou, 
Lang, \& Bolls, 2000; Hendriks Vettehen, Nuijten, \& Beentjes, 2005). Lipovetsky and Serroy (2007, see also Heijne, 2008, for a review of their work) analyse this phenomenon as an aspect of modern culture in which, as a consequence of the pervading influence of television and Hollywood-style entertainment, we experience the world according to cinematic laws (which they call "l'esprit cinema"). Reality television is an example where drama is constructed from the lives of ordinary people by methods of the cinema. We consciously or unconsciously adopt these methods when we use our own cameras and mobile phones to record things that happen around us on video and share the results on the Internet. On television, football games are turned into drama by more and more cameras, unusual camera angles, and exciting statistics. Serious political messages, such as those presented by former politician Al Gore in the documentary "An inconvenient truth" about global warming, are presented in a cinematic style to reach the audience. It has been suggested that even politicians are more sensitive to a movie from a colleague-politician using dramatic images about global warming, than by insights from science (Persson \& Calmhout, 2006).

These and many other examples show that video footage is more often than not chosen, enhanced, or specifically designed to reach the audience through evoking affective responses. This inspired us in 2000 to explore this affective property of video and to examine its impact in an educational task setting, or more specifically, to explore its potential in a web-based environment, since this appeared to be a promising medium for educational practice. The current $\mathrm{PhD}$ project was initiated at the Faculty of Educational Science and Technology's Department of Educational Instrumentation at the University of Twente in the Netherlands. In the next section the motivation and focus for this project will we presented.

\subsection{Motivation and Focus for Studying Affective Video in an Educational Web Context}

The capability of video to evoke affective responses and its impact on the individual recipient has long been acknowledged in educational practice. Classical media selection models (Bretz, 1971; Briggs \& Wager, 1981; Reiser \& Gagne, 1983; Romiszowski, 1988; see also Verwijs, 1998) often propose video as a suitable medium for affective learning goals and for motivating the learner. 
Related research in the field of educational science and technology, however, has received little attention for a long time. This is partly due to some influential educational researchers who have doubted whether media, including video, can make a difference for learning. Richard Clark is one of them. He triggered what became the media debate by his statement that media do not influence learning (Clark, 1983). Clark indicated that media are mere vehicles that deliver instruction, basing his position on the results of many media comparison studies that do not present convincing evidence that media make a difference for the effectiveness of instruction. Instead of focusing on comparing media, Kozma (1994) pointed out that the focus should be on attributes or capabilities within media and how they may be exploited to influence learning. The debate (which included contributions by Morrison, 1994; Reiser, 1994; Ross, 1994; Tennyson, 1994; and Ullmer, 1994) made Clark eventually concede that "... certain media and attributes are more efficient for certain learners, learning goals and tasks" (Clark, 1994, p. 22).

One function of visual images is to represent reality (Messaris, 1997). Visual images, such as in video, are able to do that, because they may resemble what they mean. Reeves and Nass (1996) have indicated with their "Media Equation" research, that people respond to media, including video media, and to persons and situations portrayed in media, as if they are real or non-mediated. As a natural social response, viewers tend to relate to on-screen persons and identify with them. By doing so they become involved in the situations that on-screen persons encounter. Aside from the video content, structural features of video such as the narrative structure, and audiovisual designs that create a certain atmosphere like lighting, music, and camera shots, allow viewers to experience the story. Proper use of these factors is expected to give video a strong affective potential, which may be exploited in learning situations (Koumi, 2006; Wetzel, Ratke, \& Stern, 1994).

In an educational context a relevant question is whether this affective potential of video may influence task performance. Task performance represents the purpose of all instruction: facilitating learners to acquire knowledge and skills, and performing tasks better than before the instruction. The relationship between affective responses to video and tasks has been researched in the past. For example, Alice Isen and her colleagues have conducted a series of psychological experiments that examined the impact of affective states on task performance, such as creative problem solving (e.g., Isen \& Daubman, 1984; Isen, Daubman, \& Nowicki, 1987; Isen, Johnson, Mertz, \& Robinson, 1985). 
Participants had to perform paper-and-pencil tasks or tasks with real objects after they were brought into a positive or negative affective state. Some studies used affective videos presented on television or projection screens to establish these affective states in participants (e.g., Isen, Daubman, \& Nowicki, 1987; Study 2). The results of these and comparable experiments by others (e.g., Kaufmann \& Vosburg, 1997; Study 2) suggest that video-evoked positive or negative affective states may also influence task performance in an educational setting.

At the start of our project in 2000 an intensive merging of video and the Internet was not yet within reach, simply because of limitations in computer speed, bandwidth, and video deliverance technology (see for instance Nielsen [2000] who discussed how this strongly influenced choices on whether and how to apply video). Based on the expectancy that the merging of video and the Internet would rapidly ameliorate given fast-evolving technical developments, it was decided that the exploration of video functionality under laboratory conditions was an appropriate choice to gain valuable insights for when technical constraints faded. Our interest in the affect-evoking potential of video led to the decision to explore that potential for educational purposes in web environments. The earlier mentioned studies that used affective video segments and tasks using separate and more classical media, such as print and television, were chosen as a starting point with the intention to explore whether similar effects as in these studies can be obtained in a web-based environment.

During the process of this dissertation, the role of video has changed to a large extent; it has reached the intensity that we described in the beginning of this chapter. This appears to affect educational practice where the Internet is widely used both outside and inside schools. E-school news, for instance, published a special report on how digital video is transforming American education (Jacobson, 2008). The fast increasing use of video and the related abundance of available quality materials on the Internet is putting video as an instructional tool as a mainstream feature of American education. It causes teachers to rethink their pedagogy to meet the needs of their learners for whom the cinematic culture of Lipovetsky and Serroy is part of their lives. At the university level the popular video site YouTube opened a section with university channels that gives access to large amounts of video materials, further illustrating how the use of video has also become a normal part of academic communication. These examples emphasise the relevance of the question whether responses to the affective properties of web-based video will 
influence task performance. When the project started, this question was already relevant and it is becoming even more so. Watching affective video clips from all kinds of web sites and being introduced to serious (video-based) weblearning activities are becoming more and more part of the learner's web environment.

\subsection{Main Question of the Project}

In sum, the orientation of this project is towards the use of web-based video for educational purposes with a focus on the relation between affective responses to video and task performance. Earlier studies on affect and task performance will be exploited to explore this issue in a web-based environment. The main question that will be addressed is as follows:

- How do affective responses to video influence task performance in a web environment?

The present project can be placed at the intersection where educational technology, media psychology, video design, and experimental research on affect and cognition coincide. The main components of the study-video, affective responses, task setting and the web context—are presented together in Figure 1.1.

To examine the relationships between these main components, a theoretical framework (developed in Chapter 2) provides insight into the following:

- the relationship between video and affective responses;

- the relationship between affective responses and performance in a task setting;

- the impact of the medium context, i.e., the web environment, on the affective potential of video in a task setting.

Based on research questions derived from this framework, three dedicated experiments are conducted and presented in subsequent chapters. To enable these experiments, an experimental web environment has been developed that served the purpose of presenting affective video and tasks. Additionally this environment was used to collect data from the participants in the experiments. 


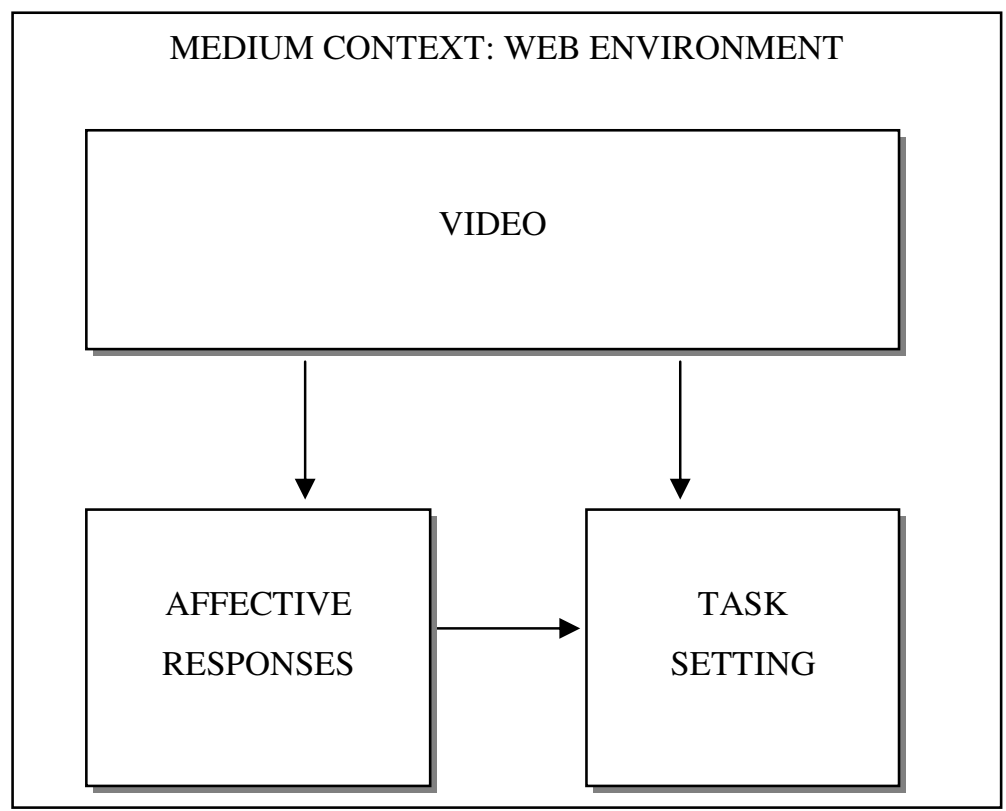

Figure 1.1: Main components of the study

\subsection{Outline of Following Chapters}

Chapter 2 presents the theoretical framework for exploring the impact of affective video on task performance. This framework results in four issues that are explored in Chapters 3, 4, and 5.

In Chapter 3 affective properties of video presentation in a web environment are explored in comparison with traditional video settings. An experiment titled "Affective responses to video: Do medium and image size matter?" is presented about the relationship between affective video content and video presentation. The results of the experiment will provide insights about the properties of webbased video as video setting for two subsequent experiments on the relationship between affective video and task performance.

In Chapter 4 the focus is on the impact of watching web video on subsequent task performance in a scenario where the tasks are not related to the content of the video. An experiment titled "Can mood-inducing videos affect unrelated (problem-solving) activities in a web-based environment?" is presented. In approach this experiment is comparable to the work by Isen and her colleagues 
who performed similar research in a traditional laboratory setting. The results of the experiment provide insights about a possible impact of affective video on unrelated task activities in a web environment.

In Chapter 5 the focus on task context is continued and extended by examining the role of video design, this time with video content that is an integral part of the content of the task. An experiment titled "What is the impact of web trigger video design on emotional responses and related task activities?" is presented. This final experiment examines affective video in a realistic web task setting.

Chapter 6 concludes the dissertation and provides a general discussion about the earlier presented experiments and to which extent they contribute to the main question of the study: how do affective responses to video influence task performance in a web environment. In addition, implications for educational practice and suggestions for further research are provided.

Note:

Because Chapters 3, 4, and 5 were originally written as separate papers, the reader may encounter some similarities and repetitions in theorising and reasoning. 


\section{Theoretical Framework}

\section{Context for Studying Presentation and Task Effects of Affective Video on the $\mathrm{Web}$}

In this chapter we build a theoretical context for the use of web-based affective video in educational task settings. We start with discussing characteristics and educational functions of video. We define affective responses, discuss affective responses to video, and discuss the related impact of audiovisual design. A discussion of the implications of affective video in a web environment then leads to the first research question that motivates the experiment reported in Chapter 3. We subsequently discuss two types of affective video in educational task settings: task-unrelated affective video (where subsequent tasks are not related to the content of the video), and task-related affective video (where tasks to be performed are about the content of the video). This part yields the research questions that are studied in the experiments of Chapters 4 and 5.

\subsection{Video and its Affective Potential in an Educational Task Setting}

In this section we introduce the characteristics of video and describe three categories of video functions and techniques that may have added value in an educational task setting. Finally, we indicate the affective potential of video for each of these categories.

Video uses two communication channels: a visual and an audio channel. It can present images (still and moving), audio (sounds, music, effects), and text (written and spoken). Basically, video consists of a continuous audiovisual stream, which makes video a time-based, externally paced medium. External 
pacing means that the medium dictates the pacing of the content presentation. The resulting linear audiovisual presentation has great potential to tell stories; video is a narrative medium.

Video, as a time-based audio-visual communication means, may be effectively exploited in educational task settings. Koumi (2006) identifies three main categories of video techniques and teaching functions for video-supported learning: assisting learning and skills development, providing (vicarious) experiences, and nurturing motivations and feelings. For each category a number of techniques determine the added value of video in a task setting. Here, they will briefly be summed up. For detailed descriptions we refer to Koumi.

In the category of assisting learning and skills development, Koumi describes characteristics that are unique to video as a time-based medium. These characteristics include diagrammatic and real-life moving pictures with synchronous sound effects and commentary, camera moves, shot transitions, visual effects, and chronological sequencing (p. 7). These characteristics allow the following techniques to support learning processes: composite-picture techniques; animated diagrams; visual metaphors/symbolism/analogy for abstract processes; modelling a process with a contrived, simplified version; illustrating abstract concepts with real-world examples; condensing time; juxtaposition of contrasting situations/processes/interpretations; narrative strength; and demonstration of skills by an expert. In this category, depending on the content, the video may evoke feelings of appreciation in the viewer when watching it.

In the category of providing (vicarious) experiences, Koumi describes how the qualities of video can show or document otherwise inaccessible phenomena and make it accessible, namely: dynamic change or movement; inaccessible places; otherwise inaccessible viewpoints; technical processes or equipment (especially complex or large-scale); showing three-dimensional objects; slow/fast motion; people or animals interacting in real-life or drama; rare or one-off events, including archive film; chronological sequence and duration of phenomena; resource material for further analysis by the viewer; and staged events/dramatized enactment.

Finally, the third category focuses on the nurturing value of video. The principal intention is to create enduring affective influences of motivations and 
feelings. The rich symbol system of video that provides substantial realism is exploited in this category. The following functions are presented by Koumi: stimulate appetite to learn; galvanize, spur into action; motivate use of a strategy by showing its success; alleviate isolation of the distant learner; change attitudes or appreciations, engender empathy; reassure, encourage selfconfidence; and authenticate academic abstractions.

The previous sections provided some insight into the potential of video for education. In the present study, the focus is on affective responses to video and how these may influence task performance in a web environment. Koumi's nurturing quality of video, which aims to evoke enduring affective responses, seems to address this affective potential of video. It is this potential that is exploited in the classical (video-induced) affect and task performance studies. The video and the task are typically not related in these studies. The provision of a vicarious experience, for example by showing situations with interacting people, is another relevant affective potential of video. In this case, such a video-presented situation could be embedded in an educational task context.

An example of one type of affective video that offers both the vicarious experience of watching people interact in real-life or drama, and the galvanizing, motivating, and empathizing quality of the nurturing category, is the trigger video, also referred to as video problem vignette (Boud \& Pearson, 1979; Cyrs, 1997; Rushby, 1987). The trigger video is a special type of video that aims to evoke affective responses with respect to a presented (problem) situation, without showing any solution. For example, imagine the following situation during a bus ride in the evening: A bus passenger, who has just entered the bus, starts to behave badly. He is shouting and makes nasty remarks to people. He looks like he is drunk. People in the bus are becoming annoyed and at a certain point the situation is no longer acceptable. What will the bus driver do? A trigger typically ends at a climax, to maximize, or trigger, affective responses in the viewer. As viewers are affected by the issue, they become motivated to reflect upon the problem situation and think about possible (re)solutions. This example shows the essential properties of a trigger video. In short, the trigger video combines the creation of affective responses with the presentation of a (problem) situation, which is part of the educational task. Moreover, in trigger videos the content (the problem presentation) and its audiovisual design are fully exploited to maximize the affective potential of the video. 
Because the internet has become more widely used in educational practice and video is no longer a limitation for the Internet, there is a renewed attention in using video for educational purposes, as recent books and journal articles show (e.g., Choi \& Johnson, 2007; Fong \& Woodruff, 2003; Koumi, 2006). Also, there have been several explorative studies dedicated to examining the pedagogical use of video in the web environment (e.g., Collis \& Peters, 2000; Young \& Asensio, 2002). These studies suggest that video in a web environment offers new forms of learning, in which images, interaction, and integration in a web environment with its communication facilities, enrich the educational task setting and the overall experience. Other studies have examined the application of streaming video in higher distance education delivered via the Internet (Reisslein, Seeling, \& Reisslein, 2005). Even though video on the Internet provides new pedagogical uses, the video functions that Koumi mentions still bring us to the key or added value of video and its affective potential that is relevant for both linear and interactive environments, such as the web. In the present study we will examine the affective responses to video and how they relate to the content and design of video.

\subsection{Affective Responses to Video: Content and Design}

In Chapter 1 it is discussed that for this study the relationship between affective video and task performance is chosen as representative for the use of affective video in an educational context. In related studies video-evoked affective responses typically concern positive or negative mood states. In other studies, the term affective response may refer to the emotional dimensions pleasure, arousal, and dominance (e.g., Kim \& Morris, 2006) or, for example, to specific feelings associated to an appraised situation, such as relief, worry, etc. (e.g., Buunk, Kuyper, \& Van der Zee, 2005). More general, affect is often used as an umbrella term to address emotions, moods, feelings, and dispositions. The previous enumeration makes clear that there is a necessity to clarify the concept affective responses and determine how it relates to the concepts emotion and mood. Therefore, these concepts will be introduced, starting with the concept of emotion.

There are at least two approaches to the study of emotions, namely the dimensional approach and the categorical approach. The categorical approach to emotion distinguishes between discrete types or categories of emotions (Ekman, Friesen, \& Elsworth, 1972; Izard, 1977; Plutchik, 1980). Categories 
may be based on, for example, facial expressions (Ekman, 1992). The distinction in dimensions of emotions was originally formulated by Mehrabian and Russell (1974) in the pleasure-arousal-dominance theory, and by Russell (1980) in the pleasure-arousal theory. These theories state that physical and social stimuli in an environment have a direct influence on the emotional state of a person, and consequently on behaviour. According to the pleasure-arousal theory, emotion consists of two dimensions. The (hedonic) valence dimension of emotion is a scale for the direction of the emotion, varying from pleasant to unpleasant. The arousal dimension represents the intensity of the emotion, ranging from low (calm and drowsy) to high (excited and energized). Some theorists also add dominance as a third dimension of emotion (Bradley, Greenwald, Petry, \& Lang, 1992; Mehrabian \& Russell, 1974). This dimension refers to the control over the experienced emotion, ranging from low (feeling small, being controlled) to high (feeling big, in control). This latter dimension is less straightforward than the other two dimensions and is often not included in studies. Media-psychological studies that are in line with the current study typically use the dimensional approach. For measuring the valence and arousal dimensions of emotion, subjective reports (verbal or pictorial semantic differential scales) and/or related physiological measures (facial muscle movement [EMG] for valence and the skin conductance response [SCR] for arousal, for example) are used.

The concept of mood is sometimes used interchangeably with emotion. Forgas (1991) summarised several theories on mood and emotion and concluded that in general moods are considered as low-intensity and relatively enduring affective states with no immediately salient antecedent cause and therefore little cognitive content (e.g., feeling good or feeling bad). Emotions would in turn be more intense, short lived, and usually have a definite cause and clear cognitive content (e.g., sadness or happiness). Frijda (1993) has suggested that emotion and mood both involve readiness; an emotion episode tends to change the state of readiness for action, and moods maintain such states and resist change (p. 125). In general, mood states of people are assessed with subjective measurements like semantic differentials scales.

In summarising the emotion and mood literature, Beukeboom (2003) concludes that the distinction between moods and emotions is not very strict. He refers to Clore, Wyer, Dienes, Gasper, Gohm, and Isbell (2001) who suggest that affective states lie on a continuum. On the one end of the continuum are the distinct and object-directed emotions and on the other end the general pleasant 
or unpleasant moods that have no specific object. According to Musch and Klauer (2003) emotion and mood are all fundamentally linked to the most basic psychological valence dimension of good versus bad, positive versus negative, approach versus avoidance. Mood states and emotional responses can range from being positive (pleasant, happy, or good) to negative (unpleasant, sad, or bad). Following the previous discussion, affective response is in the present study defined as a positive or negative valenced emotional response or mood state.

Research evidence showing that video is indeed capable of evoking affective responses, is available from different scholars. From media-psychological studies provided evidence shows that video materials evoke emotional responses in viewers (Reeves \& Nass, 1996). In one study that Reeves and Nass describe, 258 video segments of six seconds each were selected from movies, documentaries, and television programs. The segments represented a variety of emotional experiences. It appeared that these segments evoked different emotional valence and arousal responses in viewers. For example, a couple making love evoked a positive arousing response, whereas a horse-drawn procession through a cemetery was experienced as calm and negative. Experimental psychological studies have demonstrated that video segments can be effectively used to intentionally evoke discrete emotions in subjects as part of an experimental set up, such as anger, happiness, and surprise. Several authors provide information about which movie clips can be used for the elicitation of different types of emotions (Gross \& Levenson, 1995; Philippot, 1993; Rottenberg, Ray, \& Gross, 2007). Also, from the field of experimental psychology there is clear evidence that affective video materials can be effectively used to induce happy or sad mood states in viewers, as a review of different mood-induction techniques indicated (Gerrards-Hesse, Spies, \& Hesse, 1994). Together, these studies demonstrate that video content is capable of and effective in evoking affective responses in viewers, such as emotional valence responses and mood states.

Presentation properties of video may influence the strength and direction of these responses. On one hand these concern the display options for video such as the presentation medium (e.g., television or web) and the image size; on the other hand they concern the design of the video messages. In respect to design, Koumi (2006) described different audiovisual designs or production techniques, such as camera moves and shot transitions, as unique to video as a time-based medium (see 2.1). Whether a video presentation contains a captured lecture or 
an attractive Open University television course, design decisions are always part of the production process. This basic need for audiovisual design lies in the camera's limited field of view (Verhagen \& Verleur, 2007). In a long shot details are not visible; in a close-up shot the context is lacking. The audience only sees what the camera is seeing. To effectively present a story, demonstration, instruction, or any other presentation, changes of camera viewpoints are necessary to show different aspects of the subject. Millerson (1999) devotes a chapter with the meaningful title "The persuasive camera", to the way the camera can be used effectively. An effectively used camera will make the subject more appealing and will arouse the viewer's interest. This indicates that the camera does more than capturing a situation or scene-it gives an interpretation of it. Specific camera handling, such as camera shot length (e.g., close-ups to reveal a character's intention) and camera positioning in horizontal (frontal versus profile shots) or in vertical position (low-angle versus high-angle shots) may provide different impressions of the on-screen characters and the situation they are in, and, consequently, influence viewer responses.

Audiovisual design may thus have an important role in the formation of affective responses in viewers. Millerson (1999) provides some illustrative examples to demonstrate this. Pace, a video characteristic described earlier, is defined by Millerson as the "rate of emotional progression. While a slow pace suggests dignity, solemnity, contemplation, and deep emotion, a fast pace conveys vigour, excitement, confusion, brashness, etc.” (p. 431). Millerson also mentions the strong association between lighting and mood: "Through carefully chosen light direction and contrast you can change a scene's entire atmospheric impact. It can impart fun, fantasy, mystery, or dramatic tension” (p. 207). It should be noted that different audiovisual design elements together will produce the viewer responses that a director is aiming for. Take, for example, the way tension may be created in a dramatic situation (p. 429). Tension may derive partly from the dialogue, story line, and interaction between characters, but it can be strongly influenced by the way in which the subject is presented. For example, by using progressively more powerful shots (intercutting closer and closer shots, lower viewpoints) suspenseful music and effects, and information that may (or may not) be clearly presented to the audience.

The impact of audiovisual design on viewer responses has also been a subject of research (e.g., Coldevin, 1976). Without the intention of providing a complete overview, the classical studies conducted by Baggaley and Duck (Baggaley, 1980; Baggaley \& Duck, 1976) are in this context noteworthy to mention. These 
researchers systematically examined different visual designs of short television segments by conducting several small experiments. Visual elements were manipulated, such as were shot length, horizontal camera angle, vertical camera angle, background, the on-screen use of notes by a presenter, as well as combinations of some of these elements. The stimulus materials commonly showed one person, sometimes in a dialogue setting with an off-screen interviewer. These studies showed that visual design may have an impact on the evaluation of the on-screen person.

In media-psychological studies, structural or formal features of video are manipulated to examine the impact on emotional responses and other viewer responses, including information processing. For instance, studies are conducted that examined the effect of image quality (Bracken, 2005, 2006); image movement (Detenber \& Reeves, 1996; Detenber, Simons, \& Bennet, 1998; Simons, Detenber, Reiss, \& Shults, 2000; Simons, Detenber, Roedema, \& Reiss, 1999); image colour (Detenber, Simons, \& Reiss, 2000; Weisglass, 2005); pace (Lang, Bolls, Potter, \& Kawahara, 1999; Lang, Shin, Bradley, Wang, Lee, \& Potter, 2005); and image size (Detenber \& Reeves, 1996; Reeves, Lang, Kim, \& Tatar, 1999). Several studies focus on television news and the combination of formal features and emotional content that result in sensational news coverage (Grabe, Zhou, Lang, \& Bolls, 2000; Hendriks Vettehen, Nuijten, \& Beentjes, 2005).

Audiovisual design is deliberately applied as a persuasive means in commercial and political advertising. Political ads, for example, can be classified on their emotional valence: a positive ad depicts a candidate favourably, while a negative ad depicts a candidate unfavourably (Weisglass, 2005; referring to Basil, Schooler, \& Reeves, 1991). Weisglass contends that, although an "increasing body of research strongly suggests that manipulation of an ad's nonverbal elements changes the message viewers receive about the candidate, the attitudinal and emotional effects of specific alterations, remain largely unknown" (p. 3-4). One of the audiovisual elements that Weisglass manipulated in an experiment was music. He found a strong correlation between musicinduced mood and candidate evaluation: negative music lowered candidate ratings, compared to positive music and the control. For a discussion of the impact of images in the context of visual persuasion see Messaris (1997; also discussed in Borchers, 2005). The general conclusion that can be drawn from these and other studies is that video design or structural features are factors that have a strong impact on psychological responses in viewers. So, when it comes 
to examining the impact of video on affective responses, audiovisual design needs to be examined as well.

What has been presented so far shows that video can contain affective content, and that video design variables such as music, editing, camera handling, and lighting contribute to the creation and direction of affective responses to video. All the topics previously addressed were, however, coming from literature and studies that used video, television, or movies presented on television or projection screens. Whether video presented in a web environment will be able to evoke similar affective responses in viewers is not certain. When this dissertation was initiated in 2000 , a direct comparison of video-evoked affective responses in a television versus a computer/web setting had yet to be examined. Literature, nevertheless, suggests that television and computers may be perceived and deployed differently by users (Ferguson \& Perse, 2000; Nielsen, 1997, 2005). Although media uses are changing, television is still generally associated with social use and passive viewing, and the computer/Internet is associated with individual use and active searching. A difference is also that video on television is typically presented full screen while web video is usually presented on a part of the screen in a smaller, separate screen or "video window". Image size may have an effect in itself given research on image size. Larger screens appear to intensify some viewer responses (such as content evaluation) and evoked arousal level (Grabe, Lombard, Reich, Bracken, \& Ditton, 1999). The impact of image size on affective responses as defined in the present study (i.e., emotional valence responses and mood state) is less evident.

The impact of very small video image sizes on affective responses has not received much research attention. In discussing the literature on emotional and attentional responses to presentation attributes, Ravaja (2004) concludes that there is a "paucity of studies examining the influences of very small screens, although the impact of very small images may be of interest both in its own right and because of the proliferation of mobile telecommunication devices with small screens" (p. 109-110). Ravaja has conducted a series of studies that are dedicated to viewer responses to small screens. One of these studies (Ravaja, 2004) compares, for example, still versus moving video content on a small screen (52 mm width and $39 \mathrm{~mm}$ height). Taken together, these studies suggest that small video images are capable of evoking emotional responses (Ravaja, 2004; Ravaja, Kallinen, Saari, \& Keltikangas-Järvinen, 2004; Ravaja, Saari, Kallinen, \& Laarni, 2006). 
A related issue is how video design might work out under web conditions. Are there also differences with effects of audiovisual design for full screen television and does the web environment require specific forms of audiovisual design? For example, literature may suggest more close-ups when video is presented in a smaller display size, compared to a larger display size (e.g., Cennamo, 2001). Guidelines like these seem to be based rather on beliefs or common sense than on facts. Therefore, how video design might influence affective responses to video in a web environment should also be part of the project.

The uncertainties about responses to video content as part of a web page are reason enough for the present study to first address whether video in a webbased environment is experienced differently compared to a traditional video setting. Also, the project will need to examine how video presentation (image size) and design may affect viewer responses to video presented in a webenvironment.

The first step toward answering these questions is set in an experiment that focuses on the following research questions:

- RQ1: Do affective video materials produce similar affective responses (i.e., emotional valence responses and mood state changes) when delivered via a web-based environment as compared to a television setting?

- RQ2: Does video image size have an impact on affective responses (i.e., emotional valence responses and mood state changes)?

The purpose of this experiment (reported in Chapter 3) is to gather baseline information about affective responses to video in a web environment before paying attention to the affective potential of video for specific educational purposes. In the experiment affective video content (positive versus negative), medium (web versus television) and image size (small versus large) is manipulated. Affective responses, i.e. emotional valence response and mood change, are the dependent variables. The results of the experiment will provide insights about the properties of web-based video as video setting for subsequent experiments on the relationship between affective video and task performance. 


\subsection{Affective Responses to Video in an Educational Task Setting}

In the past, the importance of emotions and moods in educational settings has been largely neglected, except for two areas: emotions related to task and test anxiety and emotions related to the attribution of success and failure (see for a discussion Pekrun, 2005). Since the 1990s, however, the interest in emotions and moods has slowly grown and is currently considered an important topic for educational researchers and designers. For example, in 2005 the Journal "Instruction and Learning" devoted a complete issue to the role of emotion in learning situations with the aim to represent recent advances in this emergent field of educational research (Pekrun, 2005). The issue addressed three basic questions: (1) Which emotions are experienced by students in learning and achievement settings, and what is their phenomenology?; (2) What is the functional importance of emotions for students' interest, metacognition, problem solving, and performance?; and (3) How do students' emotions develop, what are their social and instructional antecedents, and can student emotions be fostered by modifying instruction and teacher behaviour? Together these questions imply that emotion and mood are expected to play an important role in the learning process. Even more, the second question indicates that affect may have a (functional) influence on the learning process.

For the present project this suggests that when video is applied in a task setting, the video-evoked affective responses might also influence the learning process. Basically, two situations may occur. First, when an affective video is used to present a problem situation, as to provide a (vicarious) experience in terms of Koumi (2006), the evoked affective responses might colour the way the presented problem is experienced. The affective responses to the video segment may thus influence the message processing. Second, the affective responses may also persist, or, in terms of Koumi's nurturing category, create enduring affective influences of motivations and feelings, which may, in turn, have an effect on activities presented after the video, whether these activities are related to the task or not. The notion that affective responses may have an impact on subsequent activities will be discussed in the next section. The impact of affective responses on the processing of the affective video content itself will be addressed in Section. 2.3.2. 


\subsubsection{Impact of Affective Video on Subsequent Activities}

As indicated earlier, emotions or moods may have a functional importance for various cognitive activities. This section raises the question of whether and how video-evoked affective responses may have an impact on task activities presented in the context of the video, whether they are related to the video or not. The video content is in this section treated as a black box. Only the positive or negative affective responses evoked by the video are examined.

Some general answers can be found in experimental psychological research, and more specifically within mood and cognition literature. Mood appears to have a certain impact on cognitive activities. Martin and Clore (2001) give a brief summary of research findings and indicate three main findings. First, being in a certain (positive or negative) mood leads to mood-congruent recall and evaluation. Mood congruency is the selective processing of pleasant information under a good mood and unpleasant information under a negative mood in memory and social judgment tasks (Isen, 1984). Second, being in a negative mood will lead to more systematic information processing compared to being in a positive mood. Third, being in a positive mood will lead to more flexibility and creativity compared to being in a negative mood. Although these appear to be the general effects of mood on cognitive activities, there are several theories using different concepts to explain these effects. One of the mood-cognition theories relates closely to learning processes and knowledge acquisition. Fiedler (2001) proposes two mood-dependent learning sets—namely accommodation and assimilation-using concepts borrowed from Piaget (1952).

Accommodation refers to tuning the cognitive system to fit the stimulus environment and assimilation refers to transforming external information to fit internal knowledge structures. Fiedler proposes that negative mood facilitates accommodation (avoiding mistakes, conserving input), whereas positive mood supports assimilation (inferences, interpretation). Martin and Clore (2001) provide a comprehensive overview of Fiedler's and other mood and cognition theories.

It should be noted that mood and cognition studies vary widely in the tasks that are used in their experimental setup. These may be memory tasks, social judgment and evaluation tasks, creative problem solving tasks, and persuasion tasks. Fiedler (1991) points out the importance of the type of task. According to him, tasks that involve the active generation of new information ("production tasks") are more sensitive to mood influence than tasks that involve passive 
conservation of information given ("reproduction tasks"). Mood influences should be most apparent under task conditions that leave much freedom for subjective strategies and selectivity. Therefore, the minimal experimental design has to include, next to the subject's mood and the valence of the stimuli, the productive versus reproductive nature of the task.

In the media communication and media psychology literature the impact of negative emotion and moods evoked by the (program) context on, for example, memory and evaluation of subsequent (television) content, is also an important topic. Studies basically draw upon the same mood and cognition literature, as described previously. Reeves and Nass (1996), for example, conclude that information that comes after a negatively valenced video will be better remembered (i.e., a reproduction task) than information after a positively valenced video. This effect complies with Martin and Clore's point that being in a negative mood will lead to more systematic information processing compared to being in a positive mood, and Fiedler's proposition that a negative mood facilitates accommodation, such as conserving input. However, there is a limitation to this effect. When the video evokes strong negative emotions, this may impair the processing of subsequent information for some minutes (Mundorf, Zillmann, \& Drew, 1991).

The video-evoked affective responses may also have an impact on the evaluation of the content that comes next (i.e., a production task). Assuming that the valence of the subsequent stimulus is neutral, the evoked mood state may colour the evaluation of subsequent information in a mood-congruent way. Information presented after a negative video may be evaluated negatively, whereas information presented after a positive video may be evaluated positively (e.g., Forgas \& Moylan, 1987). However, some studies show an opposite, contrast effect (e.g., Bolhuis, Heuvelman, \& Verleur, 2008; Coulter, 1998). A different situation occurs when the content after the affective video segment is not neutral, but is positively or negatively valenced. In this case a mood-consistency effect may be observed; in a negative program context, negative subsequent information is more appreciated and in a positive medium context positive information is more appreciated (e.g., Kamins, Marks, \& Skinner, 1991).

The previous discussion makes clear that the video-evoked affective responses may influence reproduction and production tasks in a different way. For examining the impact of affective video on task activities in a web 
environment, the type of task that will be examined is thus of great importance. Since it is expected that production tasks, which involve active generation of new information, are most sensitive to affective states, these types of tasks are therefore suitable for a closer examination in the present study. From a pedagogical point of view production tasks are also relevant. MacDonald, Stodel, Farres, Breithaupt, and Gabriel (2001, referring to Smaldino, 1999) indicated that the pedagogical approach is changing from didactic instruction to discovery of information. The learner-centred or constructivist approach, where the learner is encouraged to participate in and interact with the environment to construct individual meaningful knowledge, accords well with web-based learning (Jonassen, Peck, \& Wilson, 1999; MacDonald et al., 2001).

For the present study, production tasks will be taken as a starting point. The first step to building on and extending research findings from the past is to transfer earlier studies on the impact of (video-evoked) mood states on task performance to a web context. Therefore, an experiment is designed that is modelled after the earlier mentioned studies by Isen and her colleagues in which the influence of video-evoked affective states was studied on solving problems that were not related to the video content. The experiment focuses on the following research question:

- RQ3: Do mood-inducing video clips affect subsequent unrelated problem-solving activities, when both are presented in a web-based environment?

In the experiment (that is reported in Chapter 4) video content (positive versus negative) is manipulated and the performance on problem-solving tasks is the dependent variable. The results of the experiment will provide insights about a possible video-evoked mood effect on task activities in a web environment. It should be noted that findings of this experiment are based on the "pure", but artificial situation, where affective video content and subsequent tasks are not related. However, this situation may occur unintentionally in open web environments, where learners may alternate between serious work and relaxation via sites as YouTube, which includes a wide range of affective video content. Moreover, in an open environment learners may intentionally select affective video content in order to manage their mood state (Bryant \& Zillmann, 1994). 


\subsubsection{Impact on Processing the Affective Video Content}

The previous section discussed the impact of affective responses on unrelated tasks presented after the video. This section addresses the question: what is the impact of video-evoked affective responses on the processing of the affective video content itself? Answers to this question can be found in media psychology literature. In this research area, studies are conducted that examine the cognitive processing of television messages. Usually the effect of news items, political advertisements, or commercial advertisements on memory and evaluation is examined. To describe and predict how viewers process television (news) messages, the Limited Capacity Model of Lang (2000) will be used. In this model, television messages are described as an ongoing stream of audio and visual information and the viewer is perceived as an information processor. This model describes three sub processes (encoding, storage, retrieval) and two mechanisms (orienting behaviour, resource allocation) that are involved in information processing (see Grabe, Lang, \& Zhao, 2003). Orienting behaviour is an automatic, reflexive, attentional response to changes in the environment or to stimuli that people have learned signal important information. The viewer, the content, and the form of the message affect how resources are allocated to process the message.

Although people often experience television viewing as "easy" (Salomon, 1984), it is a complex task. The amount of ongoing information that one can attend to, encode, store, and at the same time retrieve in order to integrate newly presented information, is limited by the viewer's processing resources. This is partly caused by the fact that recipients will process information from the audio and visual channel of video separately (Paivio, 1986). This dual information processing may be beneficial for learning when the channels are, for a given audience, effectively combined, or may lead to cognitive overload in other situations (Mayer \& Moreno, 2003; Moreno \& Mayer, 1999). Viewers may control their information load to a certain extent. Basing decisions on their own goals and interests, the viewer may deliberately influence the proportion of resources allocated to various sub processes. However, the message content and form may automatically affect the viewer's orienting response and the amount of resources required to store and make sense of the message.

In Lang's Limited Capacity Model content and formal attributes of an affective video message can elicit emotional (arousal) responses. These emotional responses result in the automatic allocation of resources to encoding and 
storage. This may result in more attention to, and memory of the emotional (arousing) aspects in the message, and subsequently less attention and memory for other aspects of the message. In the theory of limited capacity, much attention is devoted to negatively valenced (news) messages in which arousal plays an important role. A study by Lang, Dhillon, and Dong (1995) examined the role of arousal versus valence on memory for television messages. They found that when valence (how positive or negative a message is) is controlled, arousing messages are remembered better than calm messages. However, when arousal is controlled, positive messages are remembered better than negative messages. Following Klimesch (1994), Lang (2000) assumes that memory consists of an associative network. During information processing, positive affect may activate relevant associations (cognitive schemata) in the viewer, which may support the integration of newly presented information.

The previous discussion addressed the impact of affective responses on memory for the video content, which is in fact a reproduction task. The possible impact on a production task, such as making inferences or interpretations of the video content, may differ. In this context, the affect as information theory of Schwartz (2001) is introduced. This theory proposes that people use their affective state as an information cue for the interpretation of or attitude about a given situation (i.e., a person, situation, or object): when it feels good (or bad), it is good (or bad). To create a positive association between affective response and the message content is a well known aim of political and commercial advertisements, for example. The evoked affective responses in viewers may thus act as an information cue that influences how the current video content is perceived. As discussed in Section 2.2, both content and formal features may attribute to evoking these affective responses.

In sum, for an affective video presented in a task setting, the previous discussion indicates that the evoked positive or negative affective responses may affect information processes in different ways. For reproduction tasks, such as memory for the video content, the Limited Capacity Model (Lang, 2000) suggested that negative arousing video material may lead to selective processing of the video content, with enhanced memory of negative information, at the expense of other information. However, when the impact of valence is examined and arousal is controlled, which accords with our definition of videoevoked affective responses, than memory for positive messages may be better (Lang, Dhillon, \& Dong, 1995). Positive (calm) video materials may support the integration of the video-presented information with related information in the 
associative memory network. For production tasks, such as video content evaluations, the content or message of the video may be evaluated more positively when the video evokes positive affect, whereas a video that evokes negative affect may be evaluated more negatively (Schwartz, 2001).

In Section 2.1 the trigger video was introduced that combines the affectevoking potential of video with the presentation of a realistic situation or problem. The instructional use of trigger videos is well established (Boud \& Pearson, 1979; Cyrs, 1997; Rushby, 1987), making the use of trigger video a valid option to explore the affective potential of task-related video content for this study. Moreover, trigger videos are typically presented in short segments, which make them a suitable component to an interactive web-based learning environment (Cyrs, 1997). The evoked affective responses in viewers watching a trigger video, may thus act as information cues for the interpretation of or attitude about a presented situation. A prominent variable that thereby influences the way in which video content is appreciated, is audiovisual design that-as was argued in Section 2.2-has a strong impact on psychological responses in viewers. This is a reason why designers of trigger videos are advised to employ audiovisual design or production techniques to maximize or emphasise the emotional impact of the scene (Rushby, 1987).

Taken together, trigger videos applied in a web environment may serve to explore the affective potential of video that is inherently connected to the task as a function of different audiovisual design variables.

In all, the previous discussion provides input for an experiment that focuses on the following research question:

- RQ4: Does audiovisual design influence viewer responses to (trigger) video content and related task activities, when both are presented in a web-based environment?

This experiment (reported in Chapter 5) will provide information as to whether affective video in a realistic web setting will influence task perception (trigger video content) and related activities. As dependent measures, both the impact on the video content (perception of the characters) and on the follow-up activity (the interpretation of the scene in terms of the main character's most likely course of action) will be examined. 


\subsection{Overview of the Study}

This chapter started off with the following main question for the study: "How do affective responses to video influence task performance in a web environment?" Affective response is in the present study defined as a positive or negative valenced emotional response or mood state. The previous sections examined the underlying relationships between video and affective responses and between affective responses and performance in a task setting. Also, the potential impact of the web environment as medium context on the affective potential of video in a task setting was explored. Based on the implications presented in previous sections research questions were formulated that are summarised below.

Research questions:

- RQ1: Do (positive and negative) affective video materials produce similar affective responses when delivered via a web-based environment as compared to a television setting?

- RQ2: Does video image size have an impact on affective responses?

- RQ3: Do mood-inducing video clips affect subsequent unrelated problem-solving activities, when both are presented in a web-based environment?

- RQ4: Does audiovisual design influence viewer responses to (trigger) video content and related task activities, when both are presented in a web-based environment?

In Chapter 1 a conceptual model presented indicates the main components of the current $\mathrm{PhD}$ project: video presentation, affective responses, task setting, and the web context (see Figure 1.1). The elaboration provided in this chapter leads to an extension of the model that is presented in Figure 2.1. Within each component the main variables are listed. Also, the model provides insight into the relationships between variables that are examined in each experiment. These are represented by arrows in the model.

The experiments that will be presented in detail in subsequent chapters will be briefly introduced and related to the conceptual model. 


\section{MEDIUM CONTEXT: WEB ENVIRONMENT}

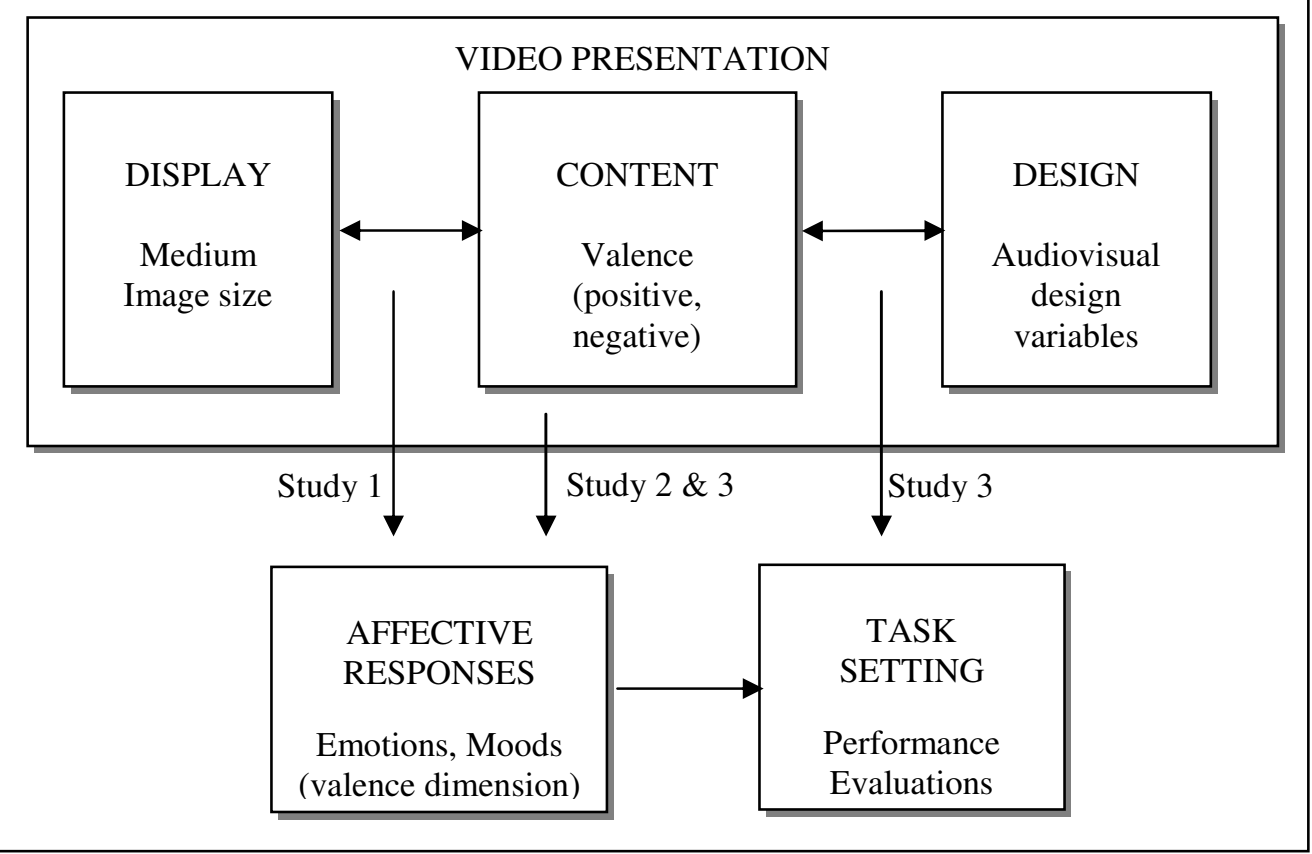

Figure 2.1: The conceptual model of the PhD project: Video Presentation, Affective Responses, and Educational Tasks

In the first experiment, presented in Chapter 3 , the issue of video presentation in a web environment is explored. A study is presented about the relationship between affective video content and video display that is titled "Affective responses to video: Do medium and image size matter?" In Figure 2.1 this study is indicated by the arrow labelled 'Study 1'. In this study RQ1 and RQ2 are addressed. Valence of video content (positive versus negative), medium (web versus television) and image size (small versus large) are the independent variables. Affective responses are the dependent variables. The results of the experiment will provide insights about the properties of web-based video as video setting for two subsequent experiments on the relationship between affective video and task performance.

The second experiment, described in Chapter 4, will focus on the task context in which web video is applied. A study is presented that is titled "Can moodinducing videos affect unrelated (problem-solving) activities in a web-based environment?" In Figure 2 this study is indicated by the arrow labelled 'Study 2'. In this study RQ3 is addressed. In the experiment video content (positive 
versus negative) is manipulated to induce a mood state (resp. positive or negative) in participants before they have to carry out problem-solving tasks that are not related to the content of the video. The performance on these problem-solving tasks is the dependent variable. The results of the experiment provide insights about a possible impact of affective video on unrelated task activities in a web environment.

Finally, in the third experiment, the focus on task context is continued and extended by examining the role of video design, this time with video content as an integral part of the content of the task. Chapter 5 presents a study that places this experiment in a realistic web task setting. In the model, this study is indicated by arrows labelled with 'Study 3' (see Figure 2). The study is titled "What is the impact of web trigger video design on emotional responses and related task activities?", and addresses RQ4. The (affective) audiovisual design of a (trigger) video is the independent variable. As dependent measures, both the impact on the video content (perception of the characters, including their affective state) and on the follow-up activity (the interpretation of the scene in terms of the character's most likely course of action) will be examined.

The results of the three experiments are discussed in the concluding chapter (Chapter 6). In that chapter we reflect on the main question of the study and to which extent we were able to provide an answer. In addition, implications for educational practice and suggestions for further research are presented. 


\section{Web Video Display}

\section{Affective Responses to Video: Do Medium and Image Size Matter?}

Most experimental studies on affective responses to video are based on traditional media, such as television or video projection screens. There is, however, a growing research interest in the impact of video delivered in contemporary applications, such as IMAX, HDTV, portable mini-TVs, web sites, mobile phones, and iPods. Does a different presentation medium change viewer responses for the same content? The study that is presented in this chapter investigates this for video presented in a web environment and aims to explore whether medium (TV or web) and image size (large or small), as a typical video display variable in the web context, have a differential impact on affective responses to (positive or negative) video content. Results obtained by a between-subjects experiment showed that medium did not have an impact on affective responses. Image size and affective content were critical, and interacting variables - emotional valence response and mood change were significantly affected by image size. Larger images accentuated the affective tone of the video content. This study further showed that affective video, as a context variable, affects responses to subsequent material.

\subsection{Introduction}

The Internet has become an indispensable medium for delivering content for various purposes and settings, such as entertainment, news, video clips, advertising and e-learning. Technical developments made it increasingly easier to use streaming as well as downloadable video. This has raised growing interest 
in applications of video within web-based environments. Almost any news, sports, or other information channel on the web now contains large amounts of clickable video and so do sites for educational purposes. This opens ways to exploit one of the most powerful characteristics of video: its potential to evoke affective responses. Viewers can identify themselves with persons on the screen in footage from real situations as well as dramatised story lines. This may evoke affective responses when viewers become involved in the depicted events (e.g., Escalas \& Stern, 2003). This study aims to explore the affective potential of video within a web environment.

Empirical evidence demonstrating that video is capable of eliciting emotional responses is mostly coming from studies using traditional media, like television and movie projection screens (Gross \& Levenson, 1995; Lang, Dhillon, \& Dong, 1995; Rottenberg, Ray, \& Gross, 2007). Some recent studies suggest that video displayed by modern devices, such as mobile phones, small LCD displays, and iPods may also evoke emotional responses (e.g., Ravaja, 2004). In all these settings, viewers tend to respond with the same basic dimensions of emotion (valence and arousal) in video-based as well as in non-mediated, real world situations (Reeves \& Nass, 1996). The (hedonic) valence dimension of emotion refers to the direction of the emotion, varying from pleasant to unpleasant. The arousal dimension represents the intensity of the emotion, ranging from low, calm or drowsy, to high, excited or energized (Russell, 1980). In addition to these "life-like" emotions, video has also shown to influence the mood of viewers (Gerrards-Hesse, Spies, \& Hesse, 1994). Emotion and mood are grounded in the same psychological valence dimension of good versus bad, positive versus negative, and approach versus avoidance (Musch and Klauer, 2003). The study of this chapter focuses on this valence dimension. It defines video-evoked affective responses as emotional responses and mood changes which vary on a valence dimension ranging from negative to positive.

When the effectiveness of web video is examined, two questions come to mind. First, is video in a web-based environment equally effective in evoking affective responses, as compared to TV settings? Second, video in web-based environments is usually presented on a part of the screen, often in a rather small "video window". Does image size have an impact on affective responses to video? Available image size research does not provide a ready answer to this latter question as it tends to focus less on the valence dimension, compared to the arousal dimension, of emotional responses. Moreover, it has been suggested that valence responses might be less sensitive to image size manipulations 
(Detenber \& Reeves, 1996). Answers to both questions are not only relevant from a theoretical point of view. They may also be relevant to web media producers, web designers, and researchers conducting web-enabled studies.

This study describes an experiment that was conducted to examine the two research questions on this matter introduced in Chapter 2:

- RQ1: Do (positive and negative) video materials produce similar affective responses when delivered via a web-based environment as compared to a television setting?

- RQ2: Does video image size have an impact on affective responses?

The potential influence of medium and image size on affective responses to video will be elaborated upon in the next two sections.

\subsection{Medium Effects}

There are indications that video materials presented in a web environment might be experienced differently than when the same materials are presented in a television setting. This has to do with how these two media are perceived and deployed by users. The results of a study by Ferguson and Perse (2000) suggest that use of the web differs functionally from television viewing. Surfing the web does not appear to be a relaxing pastime, whereas earlier studies on uses and gratifications from television viewing indicated that relaxation is an important reason for watching TV (e.g., Rubin, 1984). A major use of the web is, according to Ferguson and Perse, the acquisition of information and web materials. These activities are more goal-directed and mindful than watching TV.

Since Ferguson and Perse's publication in 2000, web use of video has evolved into a variety of forms. Classical boundaries between television and web-based computer applications are beginning to fade. Television programs, especially news programs, mimic the appearance of web pages and allow for some interaction. Other television programs allow some interaction, such as calling or sending text messages to programs for voting on participants (e.g., in a program like "Idols") or on issues presented in programs. Computers can be used as a television monitor when connected to cable TV or as video player to play back downloaded videos. Other applications, such as MSN, are more 
socially oriented, and may involve exchanging (live) video captures as well. Computers connected to the Internet can provide access to news items, video podcasts, and video collections on web sites such as YouTube or Google Video. According to Nielsen (2005), however, all these new applications have not yet changed viewers' basic perception of these media, which he described in an earlier study (Nielsen, 1997). In that study Nielsen compared television with computers along eight dimensions and identified a range of differences between these media. First, user posture during television use is relaxed and reclined, whereas with computers it is upright and straight. Second, television ambience more often implies relaxation whereas computer ambience more often implies work. Third, television use is social whereas computer use is solitary. And finally, user engagement with television is passive, whereas engagement with computers is active. Although these observations may look rather dated given the interactive forms of television entertainment just mentioned, it cannot be ruled out that Nielsen (2005) is right for the majority of television and computer use.

In sum, television and web-based applications may be perceived and experienced differently, as they imply different use patterns. However, empirical evidence for medium effects on affective video content could not be found. This emphasises the relevance of the first research question:

- RQ1: Do (positive and negative video) materials produce similar affective responses when delivered via a web-based environment as compared to a television setting?

\subsection{Image-size Effects}

The second research question (RQ2) asked whether video image size has an impact on affective responses. Grabe, Lombard, Reich, Bracken, and Ditton (1999) present a comprehensive review of image size studies. Studies on image size generally suggest that larger screens may intensify some viewer responses (for example, evaluations, memory, attention, arousal, and presence) but that this effect depends on the content of video (Grabe et al., 1999).

Video content in image size studies may vary from single shots of still and moving objects (Detenber \& Reeves, 1996), emotional expressions (Reeves, Lang, Kim, \& Tatar, 1999) and persons talking to the camera (Reeves, Lombard, 
\& Melwani, 1992; Lombard, 1995) to scenes representing TV content (Lombard, Ditton, Grabe, \& Reich, 1997), portraying sequences from action-adventure movies (Reeves, Detenber, \& Steuer, 1993) or showing point-of-view movements (Lombard, Reich, Grabe, Bracken, \& Ditton, 1995). According to Grabe et al., content might moderate the impact of screen size on viewer responses: "responses to content that emphasises the physical (spatial relationships, movement, etc.) are more likely to be influenced by screen size than responses to content that emphasises the psychological" (p. 7). As an explanation for this they suggest that image size might be less influential when content is intense in nature. With respect to affective responses, most studies on image size effects examine the impact on emotional arousal responses. Fewer studies look into the impact on emotional valence responses and mood changes. Relevant image size studies on emotional valence responses and mood changes will be discussed in the next sections.

\subsubsection{Image Size and Emotional Valence Responses}

Detenber and Reeves (1996) suggested that image size will not change valence responses because evaluations of pleasantness may depend more on what is depicted than on how it is depicted. Two studies (Detenber \& Reeves, 1996; Lombard, Reich, Grabe, Bracken, \& Ditton, 2000) measured, among other responses, emotional valence responses for different image sizes. Detenber and Reeves used 6-second single shot clips from television programs and films in which an object moved in the shot or a still shot from the same object. Although the larger size clips (90-inch, measured diagonally) were rated higher on emotional valence compared to the smaller size clips (22-inch), this effect was not significant. In another study, Lombard et al. (2000) used segments (between 40" and 1'30") portraying rapid point-of-view movement that were selected from TV and video tapes. The focus of this study was on the influence of screen size (46-inch versus 12-inch) on the viewers' experience of presencethe feeling of "being there." Along with items evaluating presence responses, emotional responses were measured via subjective reports. Results indicated that image size had no impact on emotional valence responses.

In sum, these two studies didn't find significant effects for image size on emotional valence responses. However, it is clear that the content of these clips was not selected for variance on a valence dimension, but for the potential to show moving (i.e., dynamic) content. 
In Lombard's (1995) study on direct responses to people on the screen, participants watched 24 clips of individual anchors from news broadcasts with high reward valence (positive content). Clips were between 9 and 16 seconds long. Direct responses referred to an index based on items that measured both emotional valence responses to and impressions of people on the screen. The larger screen (42-inch) resulted in significantly more positive direct responses to the people portrayed compared to the small (10-inch) or the medium screen (26-inch), with no differences between the latter two. Lombard, Ditton, Grabe, and Reich (1997) conducted a study using varying television content, including commercials, situation comedies, human drama, news, reality TV, and animation. Video content was selected to appeal to the viewers' senses (e.g., fast movement) or to evoke psychological arousal (e.g., violence). Segments ranged from a half to one-and-a-half minute. This study collected various evaluative responses measured via a questionnaire. Although emotional valence responses were not explicitly measured, an index was created from items that indirectly involved emotion (e.g., "How happy were the people?"). The large screen (46inch) yielded more intense responses than the small screen (12-inch), but this effect was not significant (also, the two screen sizes did not differ on reported enjoyment). A conclusion from this study was that psychological calm content (e.g., human drama, comedy) might be less influenced by screen size compared to dynamic arousing content (e.g., action, adventure). Video content selected for evoking affective responses on a valence dimension presumably accords with the psychological content category. Consequently, this type of video content may appear to be less sensitive to image-size manipulations.

The television or projection screen sizes in the previously described studies are in a rather limited range given the range of screen sizes in the newer technologies. Image sizes are both larger (e.g., HDTV, home theatre equipment, and IMAX theatres) and smaller (e.g., mini-TV, iPods, and mobile phones) than was usual some years ago (e.g., Bracken \& Atkin, 2004; Bracken \& Pettey, n.d.; Grabe et al., 1999; Ravaja, 2004). How these smaller image sizes affect viewer responses is of interest for web-based applications displaying video, as presented in this chapter. In discussing the literature on emotional and attentional responses to presentation attributes, Ravaja (2004) concludes that there is a "paucity of studies examining the influences of very small screens, although the impact of very small images may be of interest both in its own right and because of the proliferation of mobile telecommunication devices with small screens" (p. 109-110). Ravaja has conducted a series of studies that are dedicated to viewer responses to small screens only. One of these studies (Ravaja, 2004) 
compares, for example, still versus moving video content on a small screen that measures $52 \mathrm{~mm}$ width and $39 \mathrm{~mm}$ height. Taken together, Ravaja's studies suggest that these very small video images are capable of evoking emotional responses, including emotional valence responses (Ravaja, 2004; Ravaja, Kallinen, Saari, \& Keltikangas-Järvinen, 2004; Ravaja, Saari, Kallinen, \& Laarni, 2006). Some studies also examined very small (including iPod-like) screens), but at the same time varied the audio deliverance (speakers or ear bud headphones), which makes the interpretation of the results more difficult (Bracken \& Pettey, n.d.; Kelley, 2007).

In conclusion, although in general larger image sizes seem to intensify viewers' responses, previous studies resulted in mixed findings regarding the effect of image size on emotional valence responses to video content. It should be noted that the video content in these studies was selected for other purposes (e.g., responses to people, presence responses) and not for examining emotional valence responses by varying positive and negative video content. This latter type of content may be less sensitive to image size manipulations. Therefore, a part of the answer to RQ2 may follow from the following more detailed research question:

- RQ2.1: Does image size intensify emotional valence responses for positive and negative video content?

\subsubsection{Image Size and Mood Change}

The influence of image size on the viewer's mood state has received almost no attention in earlier studies. One exception is an unpublished dissertation study of Shapiro (1986). This study examined the effects of television screen size and sensation seeking on emotional state and physiological arousal. Participants viewed a moderately violent film on a large (72-inch), medium (19-inch), or small (5-inch) television screen. No effects were found on reported mood as a result of screen size. Shapiro concluded that image size "may not have a sufficient effect on the viewer to cause the subjective experience of intensified $\operatorname{mood}($ p. 2634)."

In a commercial context, Park, Lennon, and Stoel (2005) examined large image size (612 x 372 pixels) versus small ( $306 \times 186$ pixels), and static motion versus moving motion of product presentation in web sites on mood, perceived risk, 
and purchase intention. With respect to affective responses, this study didn't find support for the hypothesised effect for image size on mood.

To explore image size effects on mood changes as a second aspect of RQ2, the following more detailed research question was developed:

- RQ2.2: Does image size have an impact on video-induced mood changes?

\subsection{Research Method}

For this experiment a factorial design was used with three between-subjects factors: video content (positive versus negative), medium (web versus television) and image size (small versus large). Affective responses, i.e. emotional valence response and mood change, were used as the dependent variables. Gender was balanced across conditions. Each of the independent variables will be described below, followed by a description of the dependent measures.

\subsubsection{Video Content}

Affective video clips. Two video segments were selected for opposite affective content on a positive-negative valence dimension. The positive clip was a segment from the comedy movie "When Harry met Sally" (2'45"). This concerned a scene in a restaurant where an orgasm is faked. This scene is also part of a validated library of affective video segments (Gross \& Levenson, 1995) and has proven to be effective in inducing a positive affective state in a study by Roesch (1999). The negative clip was a news item about hunger in Ethiopia (2'04”), which was broadcasted by a national TV news program. This video focused on food shortage in Ethiopia and contained emotional images of suffering adults and children, as well as interviews with representatives of organizations discussing the seriousness of the situation. In a pilot study these clips produced the expected opposite responses on the positive-negative valence dimension and they produced no significant differences on arousal levels. 
Neutral video clips. In addition to these two affective video clips, two neutral clips were selected. One clip was used to create a comparable starting situation for all participants and served as a pre-test for initial mood state in order to be able to calculate a mood-change score. This clip was a segment from a documentary about birds (1'53"). The other neutral clip was a segment from an instruction video on welding (2'27"). This clip was added to make the aim of the experiment less obvious (the participants were told that the experiment was about judging video genres) and to explore the persistence of affective responses.

\subsubsection{Medium}

$W e b$. In a university computer lab individual separated computer settings were created. Seventeen inch monitors with screen resolution set to 1024 by 768 were used. Viewing distance to the monitor was approximately 20 inches (50 $\mathrm{cm})$. The affective video clip was embedded in a web page. The web page had a neutral, dark grey background and opened in an almost full screen window on top of the previous page. A play button was available to start the video clip. Each clip could only be viewed once. The video clips were located on the hard disks of the computers and played back smoothly. Headsets were used during video viewing.

Television. In a room several individual, separated TV settings were created. Participants were seated at a viewing distance of 63 inches $(1.60 \mathrm{~m})$ from a television set that included a television monitor and a separate S-VHS video player. Details of the television monitors are provided in the image size section. A dark background was created for the television monitors using a curtain. The video clips were available on S-VHS tape and could be started by a remote control. The tape was prepared in such a way that each video clip was preceded by 10 seconds of black screen and the clip's title (such as "Video clip 1") to allow enough time for the VCR to start playing and to confirm to the participants that the correct clip was started. Headsets were used during video viewing to preserve similarity of procedures between the two settings and because there were numerous television sets in one room. 


\subsubsection{Image Size within Medium Type}

$W e b$. Image size was manipulated by presenting a large or a small video display within a video window. For determining the image sizes, attempts were made to stay close to realistic settings. The web setting was used as a starting point. The viewing distance to monitors in the computer rooms is approximately 20 inches $(50 \mathrm{~cm})$. For this viewing distance a typically used video image size of $320 \times 240$ pixels (picture height $=6.6 \mathrm{~cm}$ or 2.6 inches) was chosen to represent a common situation. The viewing distance is in this case about 7.5 times the height of the picture (the resulting horizontal viewing angle* is 9.5 degrees). The larger video image size was two times the diagonal of the smaller one, resulting in another typical video image size of $640 \times 480$ pixels (picture height $=13.2 \mathrm{~cm}$ or 5.3 inches) with the same viewing distance. In this case the viewing distance is about 3.8 times the height of the picture (horizontal viewing angle: 18.6 degrees). Figure 3.1 gives an impression of the two image sizes in the web condition.

Television. Image size was manipulated by providing a large or a small television monitor. Two commonly used television monitor types were selected with picture diagonals that differed a factor 2 in size: a 14-inch (Panasonic TX14) and a 28-inch (Hantarex CT-28EQ) monitor. The horizontal viewing angle of 9.5 degrees that was applied within the web setting was also applied to determine the viewing distance for the TV settings. Based on the smaller TV screen size, this resulted in a viewing distance of 63 inches $(1.60 \mathrm{~m})$. This viewing distance was kept constant for both the small and the large television monitor.

\subsubsection{Dependent Measures}

A questionnaire containing 8 bipolar items on a 7-point semantic differential scale was used to assess the emotional valence response and mood state after viewing each video clip. The questionnaire can be found in Appendix A.

\footnotetext{
"The horizontal viewing angle is calculated as follows: Angle $=2 * \operatorname{arc} \tan [($ screen width/2)/ viewing distance)]
} 

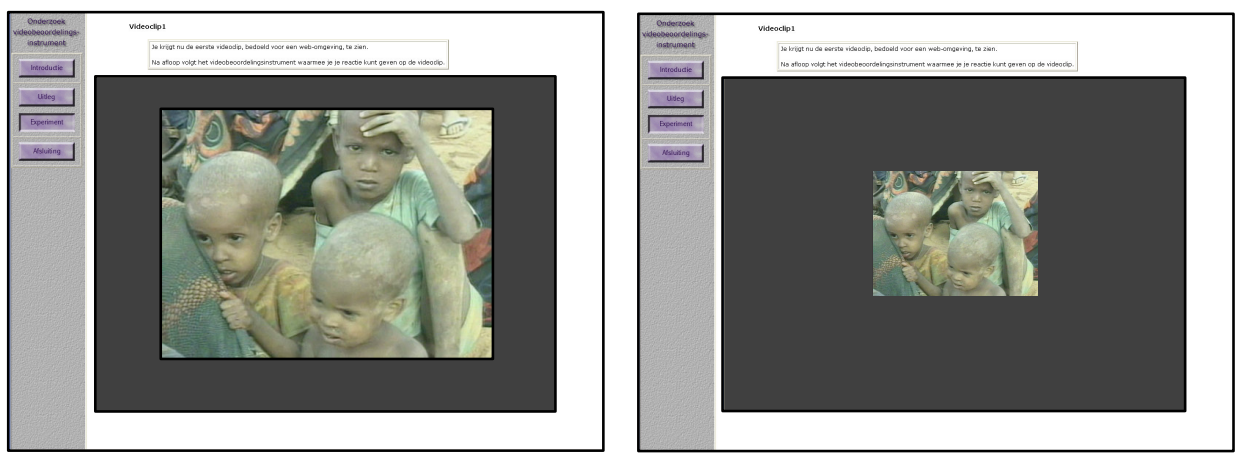

Figure 3.1. Impression of the two video image sizes in the web condition.

Emotional valence response. The feelings that the video evoked were assessed on 5 of the eight items (very unpleasant - very pleasant; very negative-very positive; very sombre - very cheerful; not at all happy-very happy; very sadnot at all sad).

Mood change. The three other items (very negative-very positive; very unpleasant - very pleasant; very sombre - very cheerful) were used to ask the participants to indicate their current mood state.

Additional arousal control measure. The questionnaire also included three items to measure elicited arousal (calm-excited; restful — restless; not at all elatedvery elated) to ensure that arousal level was not confounding the study.

In addition, other items were included to disguise the purpose of the questionnaire. In total the questionnaire contained 18 items that took about 5 minutes to fill out.

\subsubsection{Participants and Procedure}

Ninety six 1st-year university students in communication studies (65 female and 31 male, mean age $=18.6$ years) participated in the experiment. The experiment was part of an introductory course on media communication and media research. Students received course credits for their participation. The results of the experiment and students' experience in participating in it were discussed in class a month after the experiment. 
The experiment was conducted in three rooms, one for each setting: web, large TV, and small TV setting. Participants were randomly assigned to one medium (web or television) in advance. On arrival they were randomly assigned to one setting, with one video image size (large or small) and one type of video content (positive or negative). The experiment was carried out within a day in three rounds. Table 3.1 provides an overview of the treatments, groups, and cell frequencies. One of the computers in the web setting with the negative clip and the small image size malfunctioned during data collection. This caused loss of data for three participants.

Table 3.1: Overview of treatment groups and cell frequencies

\begin{tabular}{|c|c|c|c|c|c|c|c|}
\hline \multicolumn{2}{|c|}{ Medium setting } & \multicolumn{3}{|c|}{ Positive clip } & \multicolumn{3}{|c|}{ Negative clip } \\
\hline & \multirow[b]{2}{*}{ Total } & \multicolumn{3}{|c|}{ Image size ${ }^{\mathrm{a}}$} & \multicolumn{3}{|c|}{ Image size $^{\mathrm{a}}$} \\
\hline & & Total & $\mathrm{L}$ & $\mathrm{S}$ & Total & $\mathrm{L}$ & S \\
\hline Television & 47 & 26 & 11 & 15 & 21 & 12 & 9 \\
\hline Web & 46 & 27 & 14 & 13 & 19 & 13 & 6 \\
\hline Total & 93 & 53 & 25 & 28 & 40 & 25 & 15 \\
\hline
\end{tabular}

${ }^{\mathrm{a}} \mathrm{L}=$ large; $\mathrm{S}=$ small

Note. Average cell frequency is $n=12$.

In each room a trained student monitored the experiment. After a brief introduction to the experiment according to a written protocol, the students monitoring the experiment introduced the web environment that guided the experiment. The experimental procedure and the questionnaires were automated into this web-like computer-based environment. For the computer setting this was done to create the look and feel of a web environment. In order to be consistent in the overall procedure the television settings also used this environment for filling in the digital questionnaires. The web environment ran from a local drive of the computer to be independent of web-servers and to control for technical smoothness of presentations. Data were collected online using a local area network. 
The general procedure, as embedded in the web environment, was as follows. Participants started by reading a short introduction to the experiment and answering some general questions. Participants were informed that they participated in a study about cognitive and affective responses to video and for this purpose would use a new video evaluation instrument. Then the first (neutral) clip was introduced. In the web setting the video clip was embedded in the web environment. In the television setting participants had to turn their revolving chair to watch the television monitor that was set up behind them at the predetermined viewing distance of 63 inches $(1.60 \mathrm{~m})$. Participants could start the video by pressing the play button of a remote control. When the clip was finished a message to stop the tape appeared on the screen and participants turned back to the computer to continue with the procedure in the web-based environment. After watching the neutral clip, participants were asked to fill out a questionnaire. Upon completion of the questionnaire, the experimental affective (positive or negative) video clip was shown, again followed by a questionnaire. The third (neutral) clip was presented in the similar way and concluded with the same questionnaire. Finally, the participants reached the last section of the web environment which contained some concluding questions.

When a participant was done with the tasks, he or she had to wait until everyone else in the room finished their tasks. Students monitoring the experiment then concluded the session and thanked the participants. The whole procedure took about 40 minutes.

\subsection{Results}

In the following sections, first the primary data analyses are presented, followed by the results for medium effects and for image-size effects respectively. Finally, some additional analyses are provided.

\subsubsection{Primary Data Analyses}

Reliability analysis. A reliability analysis based on Cronbach's Alpha was conducted for the questionnaire items that were used for emotional valence response and mood state. It should be noted that the dependent measure for 
mood in the current study is a measure of mood change: a mood change score is the difference between two mood state scores. Based on this analysis an additive index was created for emotional valence response by averaging the questionnaires scores of the three clips (neutral clip 1, affective video clip 2, and neutral clip 3). The results are presented in Table 3.2. One of the three arousal items (not at all elated-very elated) had to be deleted to improve alpha.

Table 3.2: Mean Cronbach's alpha scores for affective responses and the arousal check variable

\begin{tabular}{llc}
\hline & & Mean \\
Variables & Items $^{a}$ & Cronbach's \\
& (Indication of question) & alpha \\
\hline
\end{tabular}

Affective responses

$\begin{array}{ll}\text { Emotional } & \text { (Which feelings does this video evoke } \\ \text { valence } & \text { in you?) } \\ \text { response } & \text { - very unpleasant / very pleasant } \\ & \text { - very negative / very positive } \\ & \text { - very sombre / very cheerful } \\ & \text { - not at all happy / very happy } \\ & \text { - very sad / not at all sad }\end{array}$

Mood state

(What is your current mood state?)

- very negative / very positive

- very unpleasant / very pleasant

- very sombre / very cheerful

Manipulation check

Arousal $^{b}$ (What is your current emotional state?)

- $\quad$ very calm / very excited

- very restful / very restless

${ }^{a}$ Items are on a 7-point semantic differential scale. ${ }^{b} .78$ refers to the resulting alpha after one item ("not at all elated / very elated") was deleted from the scale. 
Manipulation check for affective video content. The two affective video clips were compared to check whether they produced the intended opposites on the positive-negative valence dimension. A $t$ test showed that the positive clip ( $M=27.92, S D=4.81$ ) had a significantly higher (more positive) score than the negative clip $(M=9.98, S D=3.99)$, on emotional valence response, $t(91)=19.15, p=.000$. Also, in terms of mood changes, the difference between the positive clip and the negative clip was significant (respectively $M=2.09$, $S D=3.36$, and $M=-5.15, S D=3.60), t(91)=9.98, p=.000)$. This analysis confirmed that the two experimental clips were representing the valence dimension.

Multivariate analysis. A multivariate analysis of variance (MANOVA) was conducted to analyze the main effects of and interactions between the factors of video content (positive or negative), image size (large or small), and medium (web or television). An analysis method that allows for unbalanced designs with some cases in all cells (Type II in SPSS, Bryman \& Cramer, 2005) was selected. The results show a significant main effect produced by the factor video content, $F(2,84)=195.00, p=.000$, partial $\eta^{2}=.82$ ( $\eta^{2}$ : eta squared). No main effects were found for the factor medium, $F(2,84)=.86, p=.428$ and for the factor image size, $F(2,84)=1.70, p=.189$. A significant two-way interaction effect was found between the factors video content and image size, $F(3,83)=4.36$, $p=.016$, partial $\eta^{2}=.09$. There were no three-way interactions observed between the factors video content, image size, and medium. In the next sections each of the three research questions will be examined with respect to these findings.

\subsubsection{Medium Effects}

The first research question intended to explore whether medium could have an impact on the affective responses to video clips. The multivariate tests showed that the main effect of medium (TV or web) was not significant. Similarly, no significant interaction effects were found between medium and video content, medium and image size, or medium, video content, and image size. The conclusion for RQ1 is that medium did not have an impact on affective responses in this experiment: TV and web were equally effective in producing emotional valence responses and mood changes. 


\subsubsection{Image-size Effects}

Emotional valence response. The aim of RQ2.1 was to explore a potential intensifying effect on emotional valence response. The data in the upper part of Table 3.3 show that for the positive clip the larger image size produces a higher score (i.e., more positive response) and for the negative clip the larger image size produces a lower score (i.e., more negative response), regardless of the medium.

Table 3.3: Mean scores (and standard deviations) on emotional valence responses and mood changes for the video clips

\begin{tabular}{|c|c|c|c|c|c|c|}
\hline \multirow{3}{*}{$\begin{array}{l}\text { Dependent } \\
\text { variables }\end{array}$} & \multicolumn{3}{|c|}{ Positive clip } & \multicolumn{3}{|c|}{ Negative clip } \\
\hline & \multirow{2}{*}{$\begin{array}{l}\text { Means } \\
\text { Overall } \\
\end{array}$} & \multicolumn{2}{|c|}{ Image size ${ }^{\mathrm{a}}$ means } & \multirow{2}{*}{$\begin{array}{l}\text { Means } \\
\text { Overall }\end{array}$} & \multicolumn{2}{|c|}{ Image size ${ }^{\mathrm{a}}$ means } \\
\hline & & $\mathrm{L}$ & $\mathrm{S}$ & & $\mathrm{L}$ & $\mathrm{S}$ \\
\hline $\begin{array}{l}\text { Emotional } \\
\text { valence } \\
\text { response }\end{array}$ & $\begin{array}{l}27.92 \\
(4.81)\end{array}$ & $\begin{array}{l}29.88> \\
(3.57)\end{array}$ & $\begin{array}{l}26.18 \\
(5.14)\end{array}$ & $\begin{array}{c}9.98 \\
(3.99)\end{array}$ & $\begin{array}{l}9.64< \\
(4.29)\end{array}$ & $\begin{array}{l}10.53 \\
(3.50)\end{array}$ \\
\hline $\begin{array}{l}\text { Mood } \\
\text { change }\end{array}$ & $\begin{array}{l}2.09 \\
(3.36)\end{array}$ & $\begin{array}{l}3.20> \\
(3.11)\end{array}$ & $\begin{array}{l}1.11 \\
(3.33)\end{array}$ & $\begin{array}{l}-5.15 \\
(3.60)\end{array}$ & $\begin{array}{l}-5.72< \\
(4.04)\end{array}$ & $\begin{array}{l}-4.20 \\
(2.57)\end{array}$ \\
\hline
\end{tabular}

${ }^{\mathrm{a}} \mathrm{L}=$ large; $\mathrm{S}=$ small.

Note. A higher mean score represents a more positive score.

Univariate analysis of variance indicates that the interaction between video content and image size is significant for emotional response: $F(1,85)=6.45$, $p=.013$, partial $\eta^{2}=.07$. Figure 3.2 shows this accentuation (interaction) effect on emotional responses. The conclusion for the second research question is that emotional response to positive and negative video content is intensified by image size.

Mood change. RQ2.2 explored whether image size has an impact on mood change. The mean scores on mood change by the affective video clips for the large and the small image size are presented in the lower part of Table 3.3. 
Web Video Display

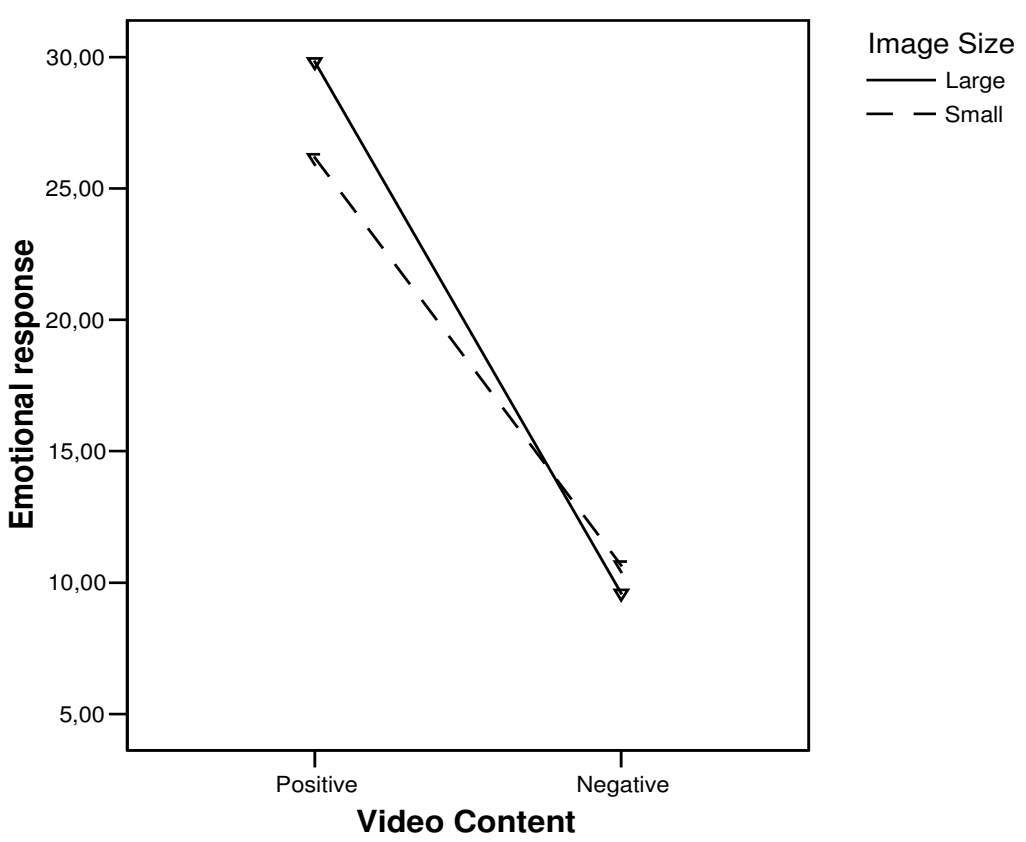

Figure 3.2. Means for emotional response by video content and image size.

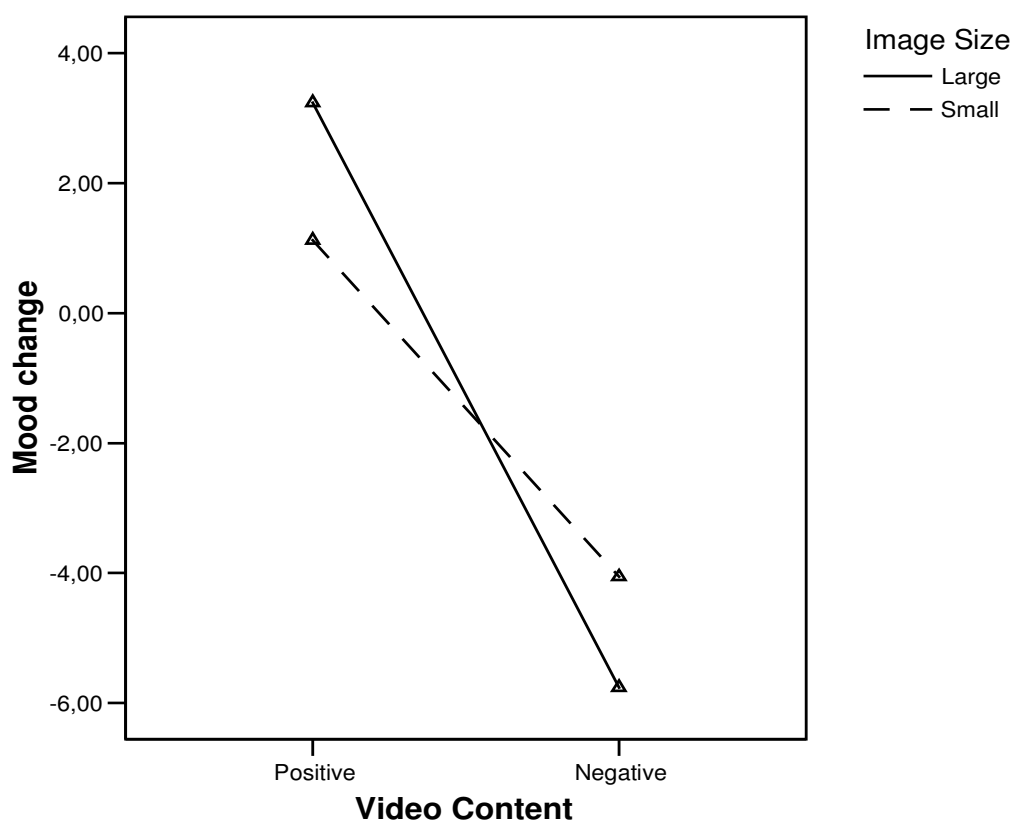

Figure 3.3. Means for mood change by video content and image size. 
The larger image size appears to accentuate the mood change in a positive direction for the positive clip and in a negative direction for the negative clip. Univariate analysis of variance shows that the interaction between image size and video content was significant for mood change: $F(1,85)=6.98, p=.01$, partial $\eta^{2}=.08$. Figure 3.3 illustrates this effect.

The conclusion for RQ2.2 is that image size has an impact on mood change in participants. This effect follows the same pattern as the effect found for emotional valence response.

\subsubsection{Additional Analysis}

Image size and arousal. The literature review showed that image size might affect emotional arousal levels. First, an analysis of variance (ANOVA) was conducted to compare the arousal levels of all treatment groups, hence for each type of affective video content (the $2^{\text {nd }}$ video clip in the experiment), in each image size, within each medium setting. The descriptive statistics for the ANOVA are presented in Table 3.4.

This analysis revealed no significant differences on arousal levels: $F(7,85)=1.81, n s$. Second, in combined medium settings, arousal levels were compared for the large versus small image sizes for each affective video clip. $T$ tests showed that there were no significant differences between the large $(M=8.6, S D=2.40)$ and the small image sizes $(M=8.25, S D=2.17)$ for the positive clip, $t(51)=.56, n s$, and also no significant differences between the large $(M=6.96, S D=2.39)$ and the small $(M=7.60, S D=2.67)$ image sizes for the negative clip, $t(38)=-.78, n s$. Arousal is not an alternative explanation for the image size effects on the dependent variables in this study.

Image size and the web. Previous image size effects were examined for combined medium settings. Since the present study started off with an interest in web video, data analyses for this setting will be provided here as well. No significant effect for image size on affective responses was observed for the negative video clip. This might relate to the cell sizes that appeared to be relatively small and unbalanced for the negative video clip in the web setting. 
Table 3.4: Mean scores and standard deviations on arousal level for affective video clips within each condition

\begin{tabular}{lcccc}
\hline Condition & N & Mean & SD \\
\hline Web & & & & \\
& Pos / big & 14 & 8.71 & 2.52 \\
& Pos / small & 13 & 8.31 & 2.06 \\
& & & \\
& Neg / big & 13 & 6.23 & 2.28 \\
& Neg / small & 6 & 6.33 & 2.66 \\
TV & & & \\
& Pos / big & 11 & 8.45 & 2.34 \\
& Pos / small & 15 & 8.20 & 2.34 \\
& & & & \\
& Neg / big & 12 & 7.75 & 2.34 \\
& Neg / small & 9 & 8.44 & 2.46 \\
Total & & & 7.89 & 2.42 \\
\hline
\end{tabular}

Note. A higher mean score represents a higher arousal level

For the positive clip a Mann-Whitney test showed a significant effect only on emotional valence response, when the large image (Mean Rank $=17.93$ ) was compared to the small image (Mean Rank $=9.77$ ), $Z=-2.87, p=.007$. This finding indicates that, at least for certain content, image size might influence affective responses in a web environment.

Post-test measures on the third clip. After participants had answered questions about the affective video clip, a neutral clip was presented. This clip served to explore the persistence of affective responses. A $t$ test was used to compare the emotional valence response to and mood change for the neutral clip after watching the positive or the negative clip. Mood change is in this case the mood state measured after clip 3 (neutral clip) minus the mood state after clip 2 (affective clip). In addition, the resulting mood state after watching clip 3 was examined in each affective video condition. The results showed that a neutral clip evokes more positive affective responses (i.e., emotional valence responses and mood changes) when following a negative clip, than when following a positive clip, as Table 3.5 indicates. 
Table 3.5: Mean scores (and standard deviations) on emotional valence responses and mood changes for the neutral (third) video clip

\begin{tabular}{llccc} 
& & \multicolumn{3}{c}{ Neutral (third) video clip } \\
\cline { 3 - 5 } $\begin{array}{l}\text { Dependent } \\
\text { variables }\end{array}$ & $\begin{array}{l}\text { Valence (second) } \\
\text { affective video clip }\end{array}$ & $\mathrm{N}$ & Mean & SD \\
\hline \multirow{2}{*}{$\begin{array}{l}\text { Emotional } \\
\text { valence response }\end{array}$} & Positive & 53 & 15.70 & 3.61 \\
& Negative & 40 & 18.88 & 4.15 \\
Mood change & Positive & 53 & -5.04 & 4.53 \\
& Negative & 40 & 3.38 & 2.73 \\
\hline
\end{tabular}

Note. A higher mean score represents a more positive score.

This effect was significant for emotional valence response, $t(91)=-3.94$, $p<.000$. Also for mood change a significant effect was found: mood changes from a preceding positive clip compared to a preceding negative clip to the neutral clip were significantly different, $t(87.20)=-11.10, p=.000$. In addition, the resulting mood states after the neutral clip were compared. The resulting mood state after the neutral clip was higher $(M=11.68, S D=2.48)$ in the version where the negative clip preceded the neutral clip, than in the version where the positive clip preceded the neutral clip $(M=10.57, S D=2.91)$. This effect was almost significant, $t(91)=-1.94, p=.056$. Apparently the two affective video clips provided a different context for the neutral clip. They had an impact on emotional valence response, mood change, and the resulting mood state.

\subsection{Discussion}

The first research question explored whether positive and negative video materials produce similar affective responses when applied in a web-based environment as compared to a television setting. Although our introduction suggested that different media may lead to different perceptions and experiences, our findings indicated that this was not the case for the affective 
responses we measured. The two medium settings were equally effective in evoking emotional valence responses and inducing mood changes in participants. There are several possible explanations why the medium setting appeared not to matter for delivering affective video content.

First, it should be noted that for reasons of experimental control the present study was designed and conducted as a laboratory study. The experimental medium settings were standardized as much as possible to avoid the influence of contaminating variables. As a consequence, concessions were made to the typical media differences that Nielsen (1997) describes. True-to-life web environments would have been more open structured and richer in content and media, with the user having complete control. True-to-life television settings would be situated in a living room, with softer lights on and often with more people present.

Second, in both settings the experimental task setting and accompanying instructions may have produced similar expectations in participants and by doing so have minimized a potential medium effect. For future studies it would be interesting to create medium settings that more closely resemble real-life medium settings.

In addition to the comparison of media, this study also examined the role of image size on affective responses to positive and negative video content. The first part of the second research question (RQ2.1) examined a potential intensifying or accentuating effect of image size on emotional valence responses and the second part (RQ2.2) examined this effect on induced mood changes. Although Grabe, Lombard, Reich, Bracken, and Ditton (1999) suggested that video with psychological content (i.e., human drama, situation comedy) was not likely to be influenced by image size, this study did find an image-size effect for this type of video content. Our findings for combined medium settings showed that larger images produced significantly more intense emotional valence responses and mood changes in participants for affective video content. Also in the web setting, this effect was clearly demonstrated for the positive video clip (a "situation comedy"). More studies are needed to gain a conclusive view of how image size might influence affective responses in web users and what is the role of video content type in this. In studies similar to ours, we would recommend systematically varying the valence dimension of the video stimuli and including affective responses as dependent measure. Given the exploratory nature of this study we used only one clip to represent each of the two opposite 
valence dimensions of video content: a positive clip (segment from a comedy movie) and a negative clip (a broadcasted news-item), each with a presentation length of around two minutes. Both clips were validated in our previous work. To examine the intensifying/accentuation effect in more depth, we suggest using a wider range of validated affective video clips that not only vary in their valence dimension but also on video genre as well as in presentation length. A presentation length of two minutes is a realistic length for a television news item or for a scene in a movie. For web applications shorter clips would also be interesting to include. Consequently, the relationship between presentation length and the mood-inducing potential of video should be examined.

Our additional analyses showed that image size did not influence the reported emotional arousal levels for the affective video content and the realistic image sizes that we used. However, a possible influence on arousal with larger screens, even for calm video content, cannot be ruled out completely. When larger screens and/or shorter viewing distances are used, more of the image is in the peripheral vision. Reeves and Nass (1996) suggested that this will cause greater arousal because "action outside of direct attention could be more consequential than action right in front of us" (p. 196). To measure arousal we used subjective reports with items on a semantic differential scale. Some studies in the past did find image size effects on arousal using physiological measures. It may be sensible to use these measures in subsequent studies or use subjective measures that correlate highly with physiological measures, such as the Self Assessment Manikin ([SAM], Bradley \& Lang, 1995).

As part of the additional analyses, we also examined the persistence of the affective responses to the video clips on a subsequent neutral video clip. The results showed that the preceding affective clip (positive or negative) influenced the emotional valence response, mood change, and the resulting mood state as measured after viewing the neutral clip. Apparently the two opposite affective video clips provided a different context for the neutral clip. After a positive clip the following neutral clip evoked more negative responses. After the negative clip more positive responses were found. 


\subsection{Conclusions and Implications for the Project}

This chapter addressed the first and second research questions that were presented in Chapter 2:

- Do (positive and negative) video materials produce similar affective responses when delivered via a web-based environment as compared to a television setting (RQ1)?

- Does video image size have an impact on affective responses (RQ2)? The experiment presented in this chapter illustrated that when affective video clips are displayed in a web environment, they induce similar affective responses as compared to a presentation in a television setting. As discussed in the previous section, it should be noted that in order to control for extraneous variables, both medium settings were set up in a controlled environment, which is different from a natural setting, like the situation at home.

The present experiment also indicated that image size matters: larger images result in more accentuated emotional valence responses and mood changes in viewers. For the web setting this effect was also demonstrated for the positive video clip. (The low number of participants in the negative condition prevented a closer examination of this effect for negative content.)

Additionally, the present chapter demonstrated that video-evoked affective responses may persist and influence a subsequent activity (i.e., response to a neutral video). The impact of prior affective video clips on subsequent activities is a relevant issue for educational web designers: video-induced emotions and moods may influence task performance in an e-learning setting. Now that we found support for the effectiveness of affective video in a web context and the accentuation of this effect caused by image size, the next step is to examine the impact of affective video on task performance in a web environment. We will explore this in the next chapter, by building upon earlier experimental studies about mood effects on task performance and transforming these to a web context.

Note:

A version of this chapter is submitted for publication: Verleur, R., Verhagen, P. W., \& Heuvelman, A. (2008). Affective video on the web: Do medium and image size matter? Manuscript submitted for publication. 


\section{Web Video Context}

\section{Can Mood-inducing Videos Affect Unrelated (Problem- solving) Activities in a Web-based Environment?}

Affective responses to video in a web environment may not be limited to the actual moment of video viewing; they may have an ongoing effect on the (task) context in which the video is used. The purpose of the study presented in this chapter was to examine whether video-induced positive and negative mood has a differential effect on subsequent activities in a web-based environment. Traditional studies from experimental psychology about mood-effects on problem-solving performance were used to explore the impact of affective video on subsequent activities in a web environment. Comparable to these studies, affective video materials were not related to following tasks. In addition, the study examined whether task conditions moderated the mood effect. The results showed that affective video clips did impact performance on an insight task but not on a divergent-thinking task. This provides evidence that affective video may influence subsequent unrelated activities in a web-based environment, but those effects seem task dependent.

\subsection{Introduction}

The strength of video lies in its realism. Videos have the potential to show scenarios and people in real-world situations as well as dramatised reconstructions. Reeves and Nass (1996) have demonstrated that viewers respond to situations depicted in videos in a similar way as they do with real experiences. This means that video has a strong affective potential, which might be pedagogically exploited when affective learning goals are at stake (e.g., 
Koumi, 2006; Wetzel, Radke, \& Stern, 1994). Affective video materials may also have educational potential when used as a context for activities. Experimental research on creative problem solving shows that affective video segments can influence performance on a subsequent problem-solving task (e.g., Isen, Daubman, \& Nowicki, 1987, Study 2; Kaufmann \& Vosburg, 1997, Study 2). The content of the video segments used in this type of experimental studies is typically not related to the task. Its purpose is to implicitly induce a positive or negative mood state in the subjects. Usually these studies are conducted in traditional settings using video monitors or projection screens to present the mood-inducing videos. These videos are followed by paper-and-pencil tasks or tasks with realia.

The notion that video materials can induce mood states is related to the way in which learners freely browse in web-based environments. Typically, people routinely browse by alternating between 'serious work' and other activities (Nielsen, 2000). Consequently, while browsing learners may encounter a wide range of materials. Learners may encounter video materials that are unrelated to their study tasks. Similar to the effects described in previously mentioned studies, these materials may evoke affective responses that unconsciously support or hinder learner performance. It is not clear whether these effects occur in a web-based environment. Studies have indicated that different media settings may be perceived and experienced differently by participants (e.g., Nielsen, 1997). The purpose of this study is to examine whether the effects of video-induced mood can be replicated in a web-based environment.

In previous chapters we defined affective responses as emotional responses and mood changes on a valence (i.e., positive to negative) dimension. As indicated, the present study is explicitly about video-induced mood. Moreover, this study is based upon previously cited experiments about mood effects on problem solving and aims to answer the following research question, which was presented in Chapter 2 as RQ3:

- RQ3: Do mood-inducing video clips affect subsequent unrelated problem-solving activities, when both are presented in a web-based environment?

Research suggests that the valence of one's mood might affect cognitive processing and task performance. Martin and Clore (2001) argue that "individuals in negative moods typically process more systematically than 
individuals in positive moods, whereas individuals in positive moods typically process with more flexibility and creativity than individuals in negative moods" (p. 2). They also discuss inconsistencies in different studies suggesting that there might be moderating or interacting variables at work (e.g., Hirt, McDonald, Melton, \& Harackiewicz, 1996).

Kaufmann and Vosburg propose that task description or conditions of problem solving tasks have this kind of moderating role (Kaufmann \& Vosburg, 1997; Vosburg, 1998; Vosburg \& Kaufmann, 1999; Kaufmann \& Vosburg, 2002). Divergent-thinking and insight tasks require different solution requirements. In a divergent-thinking task, participants are asked to generate as many solutions as possible for a given problem. Performance of this type of problem-solving task is controlled by the participants themselves according to self-selected subjective criteria. When a participant is satisfied with his or her answer, the task is finished (satisficing condition). In a typical insight task, participants are asked to propose an optimal solution to an insight problem, which often have only one solution (optimising condition). This idea of distinguishing solutions in terms of satisficing and optimising requirements was originally proposed by Simon (1956).

Vosburg (1998) proposes that a positive mood is assumed to lower and a negative mood is assumed to raise criteria for acceptable solutions. This suggests that a positive mood facilitates divergent-thinking task performance and inhibits insight-task performance while a negative mood facilitates insight-task performance and inhibits divergent-thinking task performance. Therefore, RQ3 is elaborated in the following hypotheses:

- Hypothesis 3.1: Performance on an insight task (optimising condition) is better after watching a negative affective clip than after watching a positive affective clip.

- Hypothesis 3.2: Performance on a divergent-thinking task (satisficing condition) is better after watching a positive affective clip than after watching a negative affective clip.

Table 4.1 outlines the expected effects. In the next section, the design of an experiment to test the hypotheses will be described. 
Table 4.1: Overview of hypotheses for the experiment

\begin{tabular}{lcc}
\hline $\begin{array}{l}\text { Affective video } \\
\text { content }\end{array}$ & $\begin{array}{l}\text { Insight task } \\
\text { (optimising condition) }\end{array}$ & $\begin{array}{l}\text { Divergent-thinking task } \\
\text { (satisficing condition) }\end{array}$ \\
\hline Positive & - & + \\
Negative & + & - \\
\hline
\end{tabular}

Note. + = facilitates performance; - = inhibits performance

\subsection{Research Method}

One hundred and one first-year university students in communication studies (70 female and 31 male, mean age $=18.67$ years) participated in an experiment for which they earned course credits. In a between-subjects design two affective video conditions were used: a positive and a negative condition. In each condition, the participants first encountered a neutral affective video clip. This was done to create a pre-test measure for mood, and was followed by a practice section to get participants accustomed to the experimental procedures. Then a positive mood-inducing video clip was shown in one condition and a negative mood-inducing video clip in the other condition. In each condition, the video clip was followed by two randomly ordered problem tasks: an insight task and a divergent-thinking task. The affective video clips were not related to the tasks. The video clips and problem-solving tasks are specified below. The study was balanced by keeping the number of subjects in each of the two conditions equal and subjects were blocked by gender.

\subsubsection{Video Clips}

The same mood-inducing video clips of about 2 to 3 minutes used in the experiment of Chapter 3 were used here. It concerns the positive video clip that was taken from the comedy movie 'When Harry met Sally' (2'45"). This scene is a part of a validated library of affective video segments (Gross \& Levenson, 1995). The negative video clip is a news item about hunger in Ethiopia (2'04"), which was broadcasted by a national TV news program. The neutral video segment is from a documentary about birds (1'53") and had also been shown to be effective in the study of Chapter 3 by enabling a pre-test measure for mood. 


\subsubsection{Problem-solving Tasks}

Two tasks were selected to illustrate optimising and satisficing solution requirements. A divergent-thinking task was used to exemplify a satisficing requirement, and an insight task was used to illustrate an optimising requirement. The insight task was adapted from Kaufmann and Vosburg (1997). The divergent thinking task was based on Vosburg (1998). Both tasks were originally paper-and-pencil tasks and were subsequently adapted for web use through a web-based form.

The selected insight task is the 'two-string problem' from Maier (1970). In the two-string problem, the task was to tie together two strings hanging down from the ceiling. The strings were too far apart to be reached without using tools. Participants had to solve this problem by using tools selected from a set of available items: a screwdriver, a box of tacks, and pliers. The correct solution was to tie one or each string to one of the tools (i.e., the pliers or the screwdriver), push one or both into a pendulum movement, and grab the string(s) when they are close together. The task was presented in writing with an illustration of the situation and the tools available, similar to the Kaufmann and Vosburg (1997) study.

The selected divergent-thinking task was a real-life task, referred to as the 'class problem' (Mraz \& Runco, 1994). This was a verbal description of a realistic problem that may be encountered in a classroom, written from the perspective of the problem solver. Vosburg's (1998) description of the task used was as follows: 'Rolf, a friend of yours sits next to you in the classroom. Rolf likes to talk to you and often interrupts you when you are taking notes. Sometimes he distracts you so that you are missing important parts of the lecture.' The task instruction asked the subjects to think of solutions to this problem.

\subsubsection{Web-based Environment}

An experimental web-based environment was designed to embed the video clips and problem tasks. It guided the participants through the experiment comparable to structured online learning settings. This was done to stay close to realistic learning situations. Figure 4.1 provides an impression of the look and feel of the web-based environment. The left side of the figure shows an example 
of a video window on top of a web page. This was used for playback of the mood-inducing videos. The right side of the figure shows a web page with a task (two-string problem) and the form for submitting the solution.
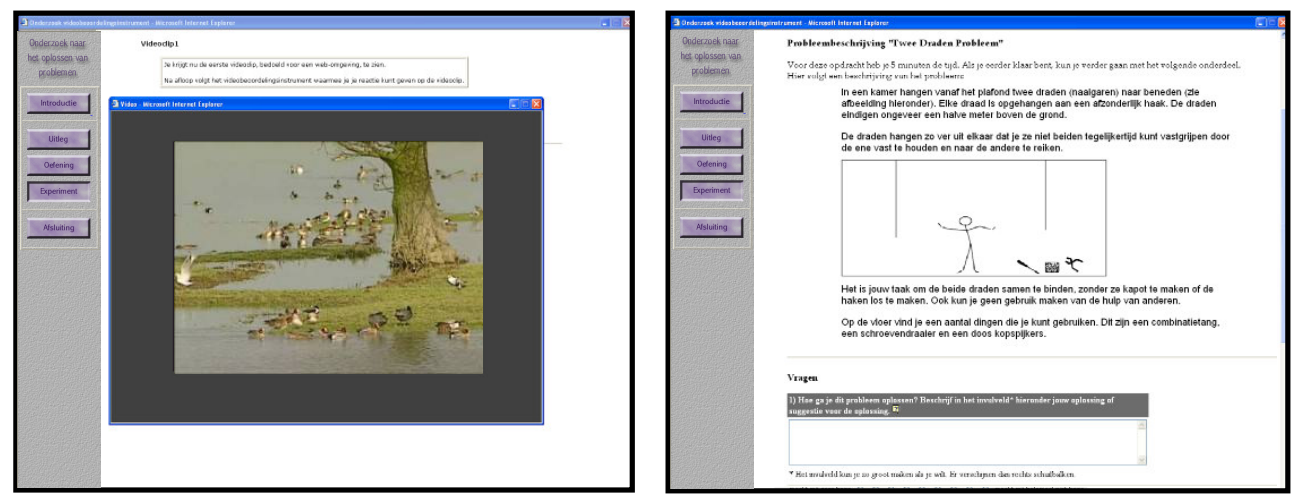

Figure 4.1: The web interface for the experiment. Left: video window. Right: task frame with the two-string problem.

\subsubsection{Instruments}

Video-induced mood assessment. A 12-item questionnaire was presented (see Appendix B). A 9-point semantic differential scale was used to ascertain whether the affect manipulation was successful ('describe your current mood' ranging from very positive to very negative). Another item was included to check whether the video clips affected the subject at similar arousal levels. This was done to ensure that arousal level was not confounding the study. Other items were included to disguise the purpose of the questionnaire.

To measure the arousal levels after watching the video, a paper-and-pencil version of the Self-Assessment Manikin (SAM) devised by Peter Lang (Bradley \& Lang, 1995) was adapted into a web version. The SAM instrument is presented in Figure 4.2. This measure uses drawings of a manikin showing emotional states. The measure was chosen because it appears to correlate highly with physiological measures of arousal by skin conductance (e.g., Lang, Bolls, Potter, \& Kawahara, 1999). Similar to the SAM-instrument procedure, all three dimensions of the SAM-scale (i.e., valence, arousal, and dominance) were embedded in the questionnaire. However, only the arousal-item, ranging from calm to excited on a 9-point rating scale (see the second row in Figure 4.2), was relevant to the present study. 
1) Geef voor elk van de drie onderstaande visuele schalen aan welke figuur het meest overeenkomt met de wijze waarop iji je nu voelt. ?

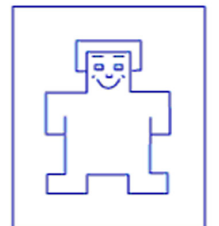

O

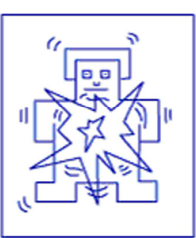

O

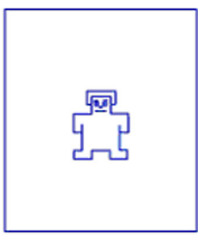

O

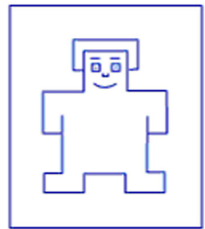

0

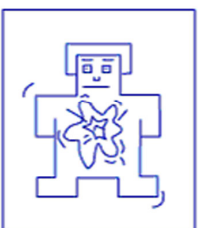

O

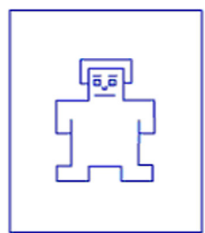

0

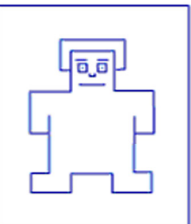

0

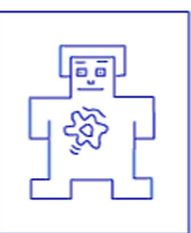

○

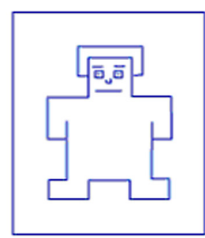

O

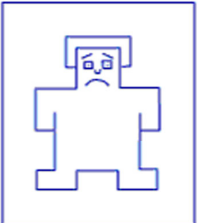

O

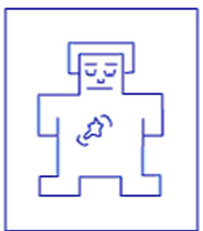

O

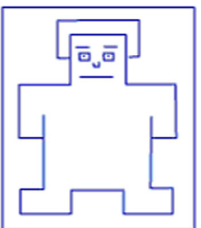

O

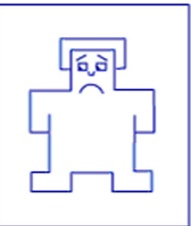

0

O

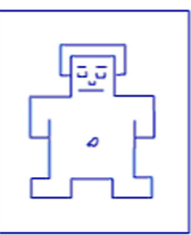

O

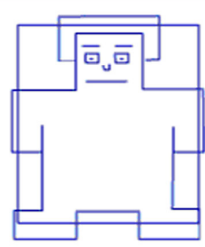

O
O

Figure 4.2: The web version of the Self-Assessment Manikin (SAM), with the arousal measure on the second row (adapted from Bradley \& Lang, 1995).

Problem-solving performance. Both the two-string and class problems used an open-answer format (fill-in fields in a web form). As in Martinsen's (1993) version of the Two-string task, two points were given for the correct solution: one point for a good try (for instance, when the tacks were used to stitch one or both strings to the wall to get them closer to each other), and no points for a wrong or no solution. Hence, the score for this task could be 2, 1, or 0 . For scoring the class problem, Vosburg's (1998) fluency criterion was used, i.e., generated solutions were counted.

\subsubsection{Procedure}

The experiment was conducted simultaneously in two computer rooms. Each room comprised 20 individual separated computer workstations with 17-inch monitors. Each session lasted approximately 1 hour and 15 minutes. After a 
brief introduction to the experiment, participants were asked to click on the icon on the monitor desktop to launch the experimental web environment. This initiated an introduction providing information on the purpose of the experiment as well as the types of questions used in the questionnaires. A subsequent practice section served two purposes. First, it supported participants in becoming acquainted with the experimental procedure. Second, it served as a pre-test measure. The practice section started with presenting the neutral affective video clip followed by a questionnaire to measure the participants' initial mood. The practice section was concluded by an example problemsolving task.

The experimental section was initiated by showing the positive or negative affective video clip. This was followed by a questionnaire to measure the videoinduced mood. Then the two problem tasks were presented in random order: half the participants first encountered the insight task followed by the divergent thinking task, while the other half received the tasks in reverse order. For each task, a maximum of 5 minutes was available to find a solution. The experiment was concluded online by thanking the participants for their participation.

\subsection{Results}

During the practice session, the initial mood ( 1 = very negative, $9=$ very positive) was measured immediately after exposure to the neutral video clip. Overall, the neutral clip resulted in a light-positive $\operatorname{mood}(n=101, M=6.32$, $S D=1.44$ ). When the two experimental conditions were compared, a MannWhitney test was used to indicate the difference between the two conditions. The difference was not significant on initial mood, $Z=-0.36, n s$. The two conditions can be considered to be homogeneous.

\subsubsection{Video-induced Mood}

Tests were conducted to measure whether the two affective video clips were effective in inducing different moods (positive vs. negative) and similar arousal levels. The mean score for the positive clip $(M=7.34, S D=1.19)$ was higher (more positive) than the mean score for the negative clip $(M=3.53, S D=1.47)$. A Mann-Whitney test confirmed that the between-subjects difference was 
significant, $Z=-8.19, p<.001$. There was also clear evidence of change in subjects' mood when comparing the measurements recorded after exposure to the neutral and the affective video clips. A Wilcoxon signed ranks test revealed that this mood change was significant both in the positive video condition, $Z=-4.45, p<.001$, and in the negative video condition, $Z=-6.13, p<.001$. A Mann-Whitney test was used to compare the arousal levels (measured by the SAM-arousal item) for the two types of affective video clips. This showed no significant difference, $Z=-0.64, n s$. In both conditions the arousal level approached the mid-point of the scale. These results confirm the findings from Chapter 3 with respect to the same clips.

\subsubsection{Insight-task Performance}

In Hypothesis 3.1 we predicted improved performance on the insight task (optimising condition) when the task was presented after the negative affective clip compared to the positive affective clip. Overall, only 20 subjects (19.8\%) found the best solution to the two-string problem. Of the 51 subjects in the negative video condition, 12 found the optimal solution to the problem, 31 had a 'good try' score, and 8 failed to solve the problem. Of the 50 subjects in the positive video condition, 8 solved the problem, 27 had a 'good try' score, and 15 failed to solve the problem. Solutions categorised under 'good try' $(f=58)$ were 'extending string or arm with tools' ( $f=25$ ), 'stitching strings to the wall or ceiling' ( $f=23$ ), or 'pushing strings into a pendulum movement without using (sufficient) weight' ( $f=10$ ). Table 4.2 summarises these results. The MannWhitney test indicated that the differences between the two conditions were significant, $Z=-1.68, p<.05$. These results confirm Hypothesis 3.1.

Table 4.2: Frequencies for solutions to the two-string problem

\begin{tabular}{lcccc}
\hline & \multicolumn{3}{c}{ Solution category } & \\
\cline { 2 - 4 } $\begin{array}{c}\text { Affective video } \\
\text { condition }\end{array}$ & $\begin{array}{c}\text { No/wrong } \\
\text { solution }\end{array}$ & Good try & $\begin{array}{c}\text { Best } \\
\text { solution }\end{array}$ & Total \\
\hline Positive & 15 & 27 & 8 & 50 \\
Negative & 8 & 31 & 12 & 51 \\
Total & 23 & 58 & 20 & 101 \\
\hline
\end{tabular}




\subsubsection{Divergent-thinking Task Performance}

In Hypothesis 3.2, we predicted improved performance on the divergentthinking task (satisficing condition), when the task was presented after the positive affective clip compared to the negative affective clip. This was measured by the number of solutions generated for the class problem. Solutions given by the subjects were categorised in order to distinguish between the different solutions/answers. The data showed that some subjects combined two solutions in one, while others presented them as two separate solutions. For example, two solutions might have been 'asking Rolf to stop talking' and 'suggest talking with Rolf during the break'. When subjects combined these two solutions into one sentence both solutions were counted. To solve the problem, subjects in the negative affect condition generated a mean number of 4.24 solutions $(S D=1.63)$. Subjects in the positive affect condition generated a mean number of 4.32 solutions $(S D=1.97)$. A $t$ test indicated no significant differences between the two conditions, $t(99)=-.24$, ns. Therefore, Hypothesis 3.2 was not confirmed during this study.

\subsection{Discussion}

In order to gain insight into the way video-induced mood might affect task performance, this study investigated whether task characteristics moderate the mood effect. Based on Kaufmann and Vosburg's studies, we devised two hypotheses. First, that problem-solving activities in an optimising task condition (i.e., the problem is solved when the solution matches the objective solution criteria) would be impacted by a negative mood. Second, that problemsolving activities in a satisficing task condition (i.e., the problem is solved when the user is satisfied about the given solution[s]) would be impacted by a positive mood. Our findings confirmed Hypothesis 3.1: viewing a negative video clip improved performance while exposure to a positive video clip reduced performance on an insight problem (two-string problem). Hypothesis 3.2 was not confirmed because the positive video clip did not support performance on the divergent-thinking task (class problem).

Reasons why the satisficing condition did not have the expected impact might be explained through a closer examination of the results. In both the positive and the negative conditions the mean number of generated solutions for the 
class problem was only four. Therefore, this task might not have been sufficiently discriminating to test the predictions of the satisficing condition.

The distinction between satisficing and optimising task conditions might still be valid and applicable to learning task settings. This can be compared to the notion of 'stop rules' (Martin, Ward, Achee, \& Wyer, 1993). Stop rules are instructions for the participants to know when they can stop working on a task; for example, when they do not enjoy it any more (cf. satisficing condition) as opposed to when they feel they have gained sufficient information (cf. optimising condition). Hirt, McDonald, Melton, and Harackiewicz (1996) investigated the role of stop rules. They found that participants in a positive mood spent more time on a task and generated more items than other participants in a satisficing condition (enjoyment-based stop rule) than in an optimising condition (performance-based stop rule). In addition, they found that regardless of the stop rules participants in a positive mood showed greater interest in a task and were more creative. It is clear that mood affects learning and, as demonstrated in our study, is dependent upon task conditions.

Traditional experimental mood studies were used as a basis for our research. Pekrun (2005) questioned the external validity of these studies: "It remains an open question to which extent the results of experimental mood research can be generalized to more intense emotions experienced in real-life academic situations outside the laboratory" (p. 501). The present study might be considered an attempt to bring traditional experimental mood studies closer to more realistic settings. Our findings support the claim that web-based environments might unintentionally become a mood-inducing context for learning and, as a result, affect task performance.

\subsection{Conclusions and Implications for the Project}

This chapter addressed the third research question that was presented in Chapter 2:

- Do mood-inducing video clips affect subsequent unrelated (problemsolving) activities, when both are presented in a web-based environment (RQ3)?

The experiment that was presented in this chapter illustrates that when affective video clips and tasks are presented in a web environment, the videoinduced mood may affect subsequent unrelated problem-solving activities. 
Furthermore, the experiment showed that effects depend on task characteristics or demands. It was found that the insight task used in the experiment was performed better when the affective video was negatively valenced, and the same task was performed worse when the affective video was positively valenced. In line with earlier experiments and insights from mood and (problem) task performance studies (e.g., Isen, Daubman, \& Nowicki, 1987, Study 2; Kaufmann \& Vosburg, 1997, Study 2) evidence was found for a taskdependent effect of video-induced positive or negative moods. It should be noted that in the present study and in the earlier experimental studies the affective videos and the tasks were not at all related in content.

However, in purposefully designed web-based learning activities, video materials are related to or integrated with the task (for example, Collis \& Peters, 2000; Verhagen, 1996). One well-known use of task-related video is the use of an affective video clip for the presentation of a realistic situation or problem. The affective reactions to such a 'trigger video' or 'problem vignette' stimulate higher order learning during the subsequent problem solving process (Cyrs, 1997). It combines the affect-evoking potential of video with the presentation of a problem, as in the present study. Next to studying task-unrelated affective video materials, as was the case in the present chapter, the next step is to explore how task-related affective video materials, such as trigger videos, might influence subsequent activities in a web-based environment. The next chapter will therefore present a study that investigates how trigger videos affect related learning activities in a web-based environment. Since trigger videos purposely exploit audiovisual design to enhance the impact of the problem vignette, the potential influence of alternate audiovisual designs of web trigger videos on subsequent activities will be taken into account as well.

Note:

A previous version of this chapter was published: Verleur, R., Verhagen, P. W., \& Heuvelman, A. (2007). Can mood-inducing videos affect problem-solving activities in a web-based environment? British Journal of Educational Technology, 8(6), 1010-1019. 


\section{Web Video Design}

\section{What is the Impact of Web Trigger Video Design on Emotional Responses and Related Task Activities?}

Audiovisual design might impact emotional responses, as studies from the seventies and eighties with movie and television content show. Will that be similar with web video content? The study presented in this chapter investigates whether audiovisual design variables may colour emotional responses to video and influence related activities in a web environment. To examine this, a video scene was designed that complies with the educational trigger video concept. A trigger video aims at evoking affective responses in viewers with respect to a presented social (problem) situation. An experiment was conducted that explored whether the manipulation of two audiovisual design variables (shot length and camera height) of a web trigger video affects how the problem situation and the characters (including their emotional valence responses and personality) are perceived. The results showed that audiovisual design did impact on these video-related task activities.

\subsection{Introduction}

Increased use of web video has caused a resurgence of interest in the use of video for educational purposes. New video applications occur as an integral part of multimedia-based teaching, videoconferencing is developed to connect people in educational settings, and video making by students is explored as a valuable pedagogy for active learning (Childs, Cuttle, \& Riley, 2007). Related video design should suit today's learners who are comfortable in image-rich environments, have a need for interactivity, are emotionally open, and show a 
preference for activities that promote and reinforce social interaction (Crittenden, 2002; Oblinger \& Oblinger, 2005). This coincides with the increased attention paid to the role of emotion in learning (Pekrun, 2005). Video's rich symbol system may serve to nurture motivations and feelings as one of the categories for video-supported learning (Koumi, 2006). This study falls in that category with an experiment that uses a trigger video as an experimental tool.

The trigger video is a special type of video that aims at evoking affective responses to a presented (problem) situation and is typically presented in short segments (Boud \& Pearson, 1979; Cyrs, 1997; Rushby, 1987). The brevity of video triggers complies with the interactive demands of today's learners who prefer brief presentations; video is best shown in short segments to maximise learner concentration (Shephard, 2003). The trigger video will not provide resolutions to a portrayed problem, but will end in a climax, in order to maximize, or trigger, affective responses in the viewer. As viewers are affected by the issue, they become motivated to reflect upon the problem situation and think about possible (re)solutions. The old "trigger film" has therefore a clear potential to revive as a "web trigger" to emotionally engage learners in an elearning environment (e.g., Elliott, Efron, Wright, \& Martinelli, 2003; Elliott \& Keppell, 2000; Keppell, Elliott, Kennedy, Elliott, \& Harris, 2003).

Designers of trigger videos are advised to employ audiovisual design or production techniques to maximize or emphasise the emotional impact of the scene (Rushby, 1987). Rushby mentions the subjective camera as an example of such an emotional technique. In that case the camera is not an objective observer, but acts as a character in the scene and is also directly addressed by other characters. The viewer may experience this as being part of the scene. In previous chapters we defined affective responses as emotional responses and mood changes on a valence (i.e., positive to negative) dimension. The present study explicitly concerns trigger video-induced emotions. In line with our definition, we will focus on emotional valence responses. For the purpose of additional analyses, arousal, as the second dimension of emotion (Russell, 1980), will be examined as well. In the experiment, audiovisual design will be manipulated to maximize the emotional impact of the trigger video.

The purpose of a trigger video is to trigger thoughts about the presented (problem) situation and the possible solutions or actions the main characters may take. The present study will therefore include tasks that examine whether 
audiovisual design has an impact on how the problem situation and the characters are perceived. One task concerns a follow-up activity in which the most likely action of the main character at the trigger climax has to be selected (an often-used task type for trigger videos). This is a measure for the interpretation of the problem situation as presented in the video scene. As discussed in Chapter 2 (Section 2.3.2) the affect-as-information theory of Schwartz (2001) proposes that people use their affective state as an information cue for the interpretation of or attitude about a given situation (i.e., a person, situation, or object): when it feels good (or bad), it is good (or bad). Emotional valence responses caused by audiovisual design may thus colour the interpretation of the scene. This will be explored in the present study. The other task concerns the evaluation of the characters. This task explores whether video design will impact how the characters, or more specifically their personalities, would be perceived. Section 5.2 will discuss in detail literature about how video design might impact person perception, including the perception of the character's emotion and personality.

In sum, this study addresses the fourth and last research question that was presented in Chapter 2 as RQ4: Does audiovisual design influence viewer responses to (trigger) video content and related task activities, when both are presented in a web-based environment? This question is studied here with a focus on emotional (valence) responses as the kind of viewer responses that are taken into account. Based on the previous discussion RQ4 is for that purpose reformulated and presented in two parts:

- RQ4.1: Does audiovisual design influence the emotional (valence) responses and the personality evaluations of characters in the web trigger video when both the trigger video and the tasks are presented in a web-based environment?

- RQ4.2: Does audiovisual design influence the perception of the problem situation, as indicated by viewer selections of (re)solutions to the problem situation that is presented in the web trigger video?

To hypothesise the potential effect of how audiovisual design may impact the perception of the characters involved, including their emotional responses (RQ4.1), we will borrow a theoretical framework from interpersonal theory that is presented in the next section and relate this to audiovisual production techniques. Furthermore, we will build on earlier experimental research on the effects of audiovisual design of the television image on personal perception 
(e.g., Baggelay, 1980). RQ4.2 will be explored in the experiment using the follow-up activity mentioned previously in which the most likely action of the main character at the trigger climax has to be selected.

\subsection{Dimensions in Portraying Social Interactions}

When, as is the case in the present study, the trigger video content contains a social interaction between two persons, interpersonal theory may provide some helpful insights in selecting appropriate audiovisual design techniques for portraying such a social situation. Interpersonal theory proposes that the way people relate to each other can be described in terms of their social space along two dimensions or axes. Birtchnell (1996) distinguishes two axes of humanrelatedness. The horizontal axis describes the relative distance (distance/closeness) between persons. Other scholars refer to this as the friendliness or affiliation dimension (Argyle \& Dean, 1965). The vertical axis refers to the relative strength (upperness/lowerness) of the persons involved. This is comparable to the dominance dimension. Figure 5.1 shows these social axes (adapted from Birtchnell, 1996). These axes or dimensions of social interaction are used in the present study as a starting point to discuss relevant camera manipulations for portraying social interactions.

\section{UPPERNESS}

(RELATING FROM A POSITION OF RELATIVE STRENGHT)

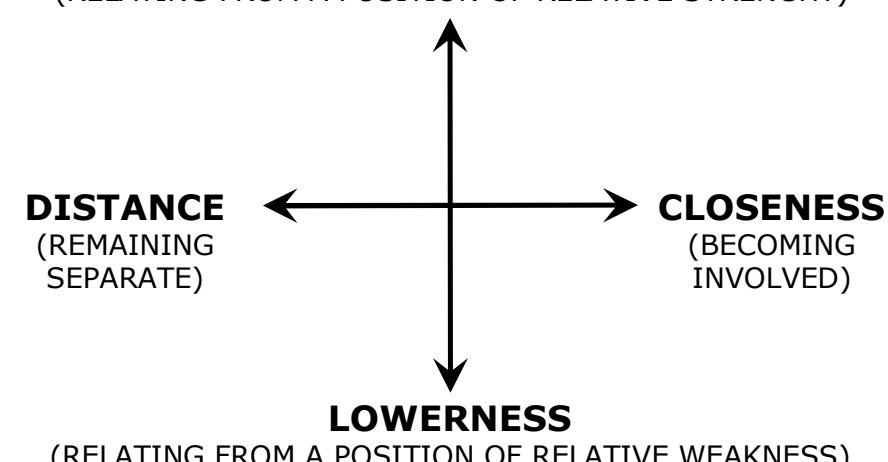

Figure 5.1: Dimensions of social interaction (adapted from Birtchnell, 1996) 


\subsubsection{Horizontal Social Distance, Camera Shot Length, and Person Perception}

The horizontal axis of human relatedness ranges from remaining separate to becoming involved (Birtchnell, 1996). Physical distance between persons can vary along a continuum from far away to extremely close. Hall (1966) distinguished four specific ranges of formality in face-to-face interaction distances: Intimate: up to 18 inches; Personal: 18 inches to 4 feet; Social: 4 to 12 feet; and Public: 12 to 25 feet. Meyrowitz (1986, in Messaris, 1997) argues that the viewing distance in visual media is comparable to interpersonal distance in real-world interaction. Hall's degrees of formality can also be found in the camera's shot length: the long shot signifies the impersonal (public), while the medium shots, the social mode, and close-ups signify intimate or personal modes (Chandler, 2000; Kress \& van Leeuwen, 1996).

In real-life interactions, reducing the interaction distance between persons will have an impact on person perception. Personal space violations may be considered as either positively or negatively valenced. This depends on the degree of positive or negative regard one has for the other person in question and on the interpretation and evaluation of the violation itself (Burgoon, 1978; 2001). When the interaction is perceived as positive, a closer distance may result in greater interpersonal attraction and more positive evaluations, whereas when the interaction is perceived as negative, this will have a negative impact on interpersonal attraction and evaluations (Schiffenbauer \& Schiavo, 1976). Depending on the interaction context (being negatively or positively valenced), a close-up shot may have two potential effects: a threatening effect or an intimacy effect (Persson, 1998). When the distance between persons is reduced, this may also change the impression of someone's emotional state. In describing nonverbal communication cues in social interaction, Argyle (1967) has pointed out that the face and body reveal different aspects of a person's emotions. Whereas bodily gestures and posture provide information about a person's emotional arousal state, facial expressions reveal the type of emotion better.

Shot length and perceived emotion. Following Argyle (1967), close-up and medium shots also differ in the emotions they portray. While a medium shot might show more about a person's tension (posture, gesture, etc), a close-up shot is focused on and limited to detailed information of facial emotional expressions (see Argyle, 1967). Unfortunately, empirical studies that have 
examined these claims are scarce. Baggaley (1980) conducted a series of experiments in which he examined the impact of visual structure of TV images on psychological responses. In one experiment he varied both the shot length (medium shot versus close-up shot) and the performer valence (friendly versus angry) of an on-screen speaker. Regardless of the performer valence, the speaker was rated as significantly less tense and nervous in the close-up version than in the medium shot. In line with Argyles notion, Baggaley suggested that the performer's hands in the mid shot versions "amplified his apparent tension even when, in the "friendly" condition, it was minimal" (p. 71). Baggaley refers to a comparable study of Wurtzel and Dominink (1971-72) with similar findings for shot-length manipulations. Like real-life interactions, a close-up shot may have two potential effects: a threatening effect in a negatively valenced interaction context, or an intimacy effect in a positively valenced interaction context (Persson, 1998). In an experimental study, Reeves, Lombard, and Melwani (1992) varied the perceived distance between viewer and video portrayals of persons with three within-subject factors: image size, viewing distance, and camera shot length. Following Schiffenbauer and Schiavo (1976), they hypothesised that when social distance is shortened, the intensity of the responses will increase. The study showed that perceived emotional valence responses were intensified: on-screen persons were judged more extreme on perceived happiness/sadness in close-up shots compared to long shots.

Shot length and perceived personality. Reeves, Lombard, and Melwani (1992) found that close-up shots, compared to long shots, resulted in more extreme evaluations (i.e., more positive or negative) of the persons portrayed. Referring to early studies of Cobin and McIntyre (1961) and McCain and Repensky (1972), Grabe, Zhou, and Barnett (1998) come up with comparable conclusions. Interestingly, although a trend in the hypothesised direction was observed, the Reeves et al. study only produced a statistically significant intensification effect for the shot-length manipulation-not for the viewing distance and image size manipulation.

Conclusions for the impact of camera shot length on dependent measures. Based on the previous discussion, shot length manipulations may have the following impact on the perceived emotional valence responses and perceived personality of a portrayed person in a web trigger video: closer shots will intensify both emotional valence responses and personality evaluations, compared to medium shots. 


\subsubsection{Vertical Social Distance, Camera Height, and Person Perception}

The vertical axis in interpersonal relationships indicates the relative strength between interacting persons, or their dominance (Birtchnell, 1996). Vertical height or spatial elevation has been identified as the most important spatial contrast that expresses social dominance or power (Schwartz, Tesser, \& Powell, 1982), where "higher than" is seen as more dominant. Schwartz et al. refer to scholars like Allen (1972) and Argyle (1967) who have explained this effect by comparing it to the human experience of social and status inequality in the parent and child relationship. The real-life effects of the vertical axis also play a role when it comes to video recordings of persons interacting. Camera height, also referred to as vertical camera angle, can be employed to signify the social power or relative strength of mediated persons (Millerson, 1999). Camera height is one of the production techniques that received considerable attention in empirical research. Studies on the effect of camera height on person perception generally look at effects on the perceived dominance and emotional state of the portrayed person or at the perceived source credibility and attraction.

Camera height and perceived emotion. Some studies suggest that the viewing angle may affect the perception of the portrayed person's emotions. Kappas, Hess, Barr, and Kleck (1994) found that faces were perceived as more positive and less negative when seen from below, whereas faces seen from above were perceived as less positive and more negative. Comparable effects were found in a study of Mignault and Chaudhuri (2003). They compared the effects of raised and bowed heads. A raised head was seen as happier (and more dominant) and a bowed head was perceived as sadder (and submissive). Apparently the "angle of regard" shows different facial expressions which leads to different attributions of a person's emotion.

Camera height and perceived personality. Shoemaker (1964, cited in Kraft, 1987, p. 292) examined the effect of low, eye level, and high camera angles. Male models photographed from a low angle were evaluated as being more positive, more active, and more potent compared to high-angle photographs. A study by Mandel and Shaw (1973) found comparable results.

The effect of camera angle on person perception is commonly examined for one on-screen person. In a study conducted by Kraft (1987), effects of camera manipulation on the perception of $t w o$ characters is examined. The stimuli in 
this study were six picture stories that contained four stills each. Camera angle affected the subjects' evaluations of physical and personal characteristics of the portrayed persons. There were three different camera angle versions for the two characters in each picture story: a low/high-angle, an eye/eye-angle, and a high/low-angle version. From the low/high-angle to high/low-angle version the ratings for the two characters in the stories reversed for 5 out of 6 scales: short/tall, weak/strong, afraid/unafraid, timid/bold, and passive/aggressive (the good/bad scale was the exception). Kraft (1987, p. 295) concludes that the "camera angle strongly defined the relative roles of the two characters in each story". Studies concerning camera angle effects on credibility and interpersonal attractiveness suggest that a higher angle may result in higher credibility perceptions, like higher sociability ratings (McCain, Chilberg, \& Wakshlag, 1977, Study 1) and higher likeability ratings (Gaudino, 1988). It has been suggested that a high camera angle may make the portrayed person appear more approachable or friendly (Gaudino, 1988; McCain et al., 1977).

McCain et al. (1977) argued that video normally does not consist of a single shot from one camera perspective. Therefore, they manipulated both camera height and shot length to explore the effects of a montage or sequence of varying camera angles. The most frequently used angle of the sequence was labelled the preponderant angle. Again, higher angles made the on-screen person seem more sociable. Interestingly, this study also examined the effect of camera angle on character perception. A higher angle resulted in higher (more positive) character scores, as measured by the following characteristics: selfish-unselfish, sympathetic-unsympathetic, kind-cruel, and virtuous-sinful. This effect, however, only occurred in the medium shot version, thus demonstrating an interaction effect between camera angle and camera shot length.

\section{Conclusions for the impact of camera height (vertical angle) on dependent} measures. Based on the previous discussion, camera height manipulations may have the following impact on the perceived emotional valence responses and perceived personality of a portrayed person in a web trigger video. Compared to low shots, high shots may make the facial expressions appear more negative and less positive (e.g., sadder); compared to low shots, high shots may make a portrayed person appear more submissive (and more credible and attractive; i.e., more approachable). The interaction between camera height and shot length may be exploited to create the strongest impact on character perception. For example, a high, medium shot may result in a more positive (e.g., sympathetic) character perception. 


\subsection{Implications for the Present Study}

The introduction section of this chapter was concluded with the presentation of two research questions, of which the following research question was the first:

- RQ4.1: Does audiovisual design influence the emotional (valence) responses and the personality evaluations of characters in the web trigger video when both the trigger video and the tasks are presented in a web-based environment?

Based on the literature review in Section 5.2, it is expected that horizontal and vertical social distances will have an impact on the perception of the characters' relative roles in a trigger video. It can be assumed that camera manipulations relating to the horizontal axis (shot length) and to the vertical axis (camera height) influence person perception, such as perceived emotional valence responses and perceived personality of on-screen characters. To hypothesise specific effects of audiovisual design for a given trigger video, different versions of the trigger video need to be defined.

For the present study, a trigger video is produced in three versions that are tested in an experiment for their impact on the perception of the problem situation and on emotional valence and personality perceptions. The portrayed dialogue scene shows a main character expressing more and more negative emotions as the story develops (see 5.1 for more script details). The first version will explore the impact of horizontal distance by manipulating the camera shot length: to stimulate involvement with the main character and to clearly show his emotions, close-up shots will be exclusively used for this character. This version will be labelled the close-up (or proximity) version. The second version will combine camera height with shot length to maximise the social inequality between the two on-screen characters (following McCain et al., 1977). This version is labelled the dominance version. The third or neutral version serves as a standard for comparison: both characters are similarly portrayed in medium shots, indicating the social mode (Hall, 1966) and are shot at eye-level, indicating status equality.

For these three versions of the web trigger video (i.e., the close-up, dominance and neutral version), the following is hypothesised for ratings on perceived emotional valence and on perceived personality of on-screen characters (hypotheses numbered relative to RQ4.1): 
Close-up (proximity) version:

- Hypothesis 4.1.1: Compared to the neutral version, the close-up version will lead to more extreme emotional valence responses as a result of the intensifying effect of close-up shot. The main character, who expresses a negative emotion, will be perceived as experiencing more negative emotions than in a neutral version.

- Hypothesis 4.1.2: Compared to the neutral version, in the close-up version the main character will be rated more extremely on perceived personality (as result of the intensifying effect of close-up shots).

Impact of audiovisual design on perception of relative roles:

Although the second character is in both versions portrayed in medium shot length, the close-up shot for the main character in the close-up version may also have an impact on perceptions of the second character, possibly affecting their relative roles. This leads to the following research question that is derived from RQ4.1:

- RQ4.1.1: Does the close-up version result in different perceptions of emotional valence responses and personality of the second character, compared to the neutral version?

Dominance version:

- Hypothesis 4.1.3: Compared to the neutral version, the dominance version will have the following impact on perceptions of the main character's emotional valence responses (in high-angle medium shots) and of the second character's emotional valence responses (in low-angle close-ups): (a) the main character will be rated as more negative (sad) and (b) the second character will be rated as more positive (happy).

- Hypothesis 4.1.4: Compared to the neutral version, the dominance version will have the following impact on perceptions of the main character's personality (in high-angle medium shots) and the second character's personality (in low-angle close-ups): (a) the main character will be rated more submissive and more positive on personality evaluations and (b) the second character will be rated as more dominant and more negative on personality evaluations. 
Impact of audiovisual design on perceived problem situation:

As indicated in the introduction of this chapter, RQ4.2 was formulated as follows:

- RQ4.2: Does audiovisual design influence the perception of the problem situation, as indicated by viewer selections of (re)solutions to the problem situation that is presented in the web trigger video?

\subsection{Research Method}

For this experiment a one factor between-subjects experimental design is used with audiovisual design (camera manipulation) as the factor. One hundred and twenty-three university students ( 66 female and 57 male, mean age $=20.6$ years) participated in this experiment. Gender was balanced across conditions. The experiment was part of a course that introduces the field of media psychology. The results of the experiment were discussed in class a month after the experiment. First, a description of the trigger video story will be given below. Subsequently, a description of the independent variable and the dependent variables will be provided.

\subsubsection{Trigger Video Story}

In the introduction of this chapter and in Section 5.2.3., the trigger video for the experiment was briefly introduced. In this section a more detailed description will be given. The trigger video was based on a written real-life problem vignette (Mraz \& Runco, 1994) in which someone is distracted by a friend during a lecture class. For practical reasons the class setting was changed into a library setting. The trigger video opens with the main character, a male student named Ralph, who is studying in a quiet study (library-like) environment. Then the second character Ron, a friend of Ralph, enters the room very enthusiastically and loudly sits down with him. Ralph says hello, but informs Ron that he is studying for an exam. Ron initially appears to accommodate him, but continues to talk to him and even comes up with photos of a field trip they recently made. Ron's behaviour clearly disturbs Ralph more and more. The story ends at the climax of Ralph's rising irritation, at the point 
where he is about to (re)act. The way he will respond is left open to the interpretation of the viewer. In Appendix $C$ the outline of the trigger story is presented.

\subsubsection{Independent Measure: Camera Manipulation}

As mentioned in Section 5.2.3, the story was developed into the three video versions for the experiment: the close-up (proximity) version, the dominance version, and the neutral version. All versions had a presentation length of approximately 50 seconds and started with a long shot to establish the library setting, showing the main character (Ralph) studying at a table. From the moment that the second character (Ron) enters the scene, the versions are different in camera manipulations. All versions end with a shot of the main character that is about to (re)act. The neutral version uses medium shots for both characters in the entire scene that follows. The close-up version uses close-ups only for Ralph, to emphasise his emotional expressions. In this case, the final shot is an extreme close-up. The dominance version uses high-angle medium shots for the main character to emphasise his submissiveness/weakness (and credibility and attractiveness). This version uses low-angle close-up shots of the second character to emphasise his dominance/strength, both by angle and shot length. In the production of the video clips, the neutral version was the basic version. For the close-up version, medium shots from the main character in the basic version were removed and changed for inserts of close-up shots of the main character. For the third version all shots had to be changed in order to enable the camera angle manipulation for both the main and the second character. In sum, the independent variable camera manipulation consists of a neutral, a close-up (i.e., camera shot length), and a dominance (i.e., combined camera angle/shot length) version. Figure 5.2 presents an overview of typical shots used in the three web trigger video designs.

\subsubsection{Dependent Measures}

As previously discussed, the dependent measures in this experiment are the emotional (valence) response and the perceived personality of the characters in the trigger video (see RQ4.1), and the perceived problem situation (see RQ4.2). 
Version:

Neutral

Close-up

Dominance

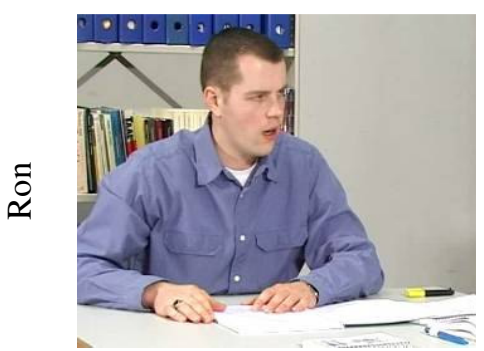

MS

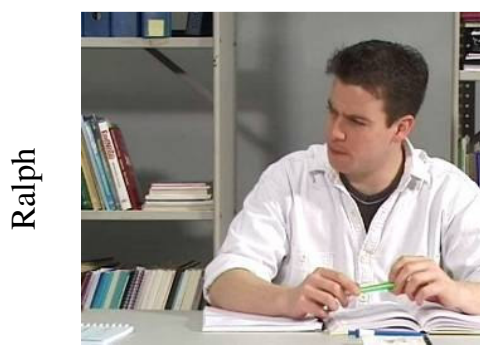

MS

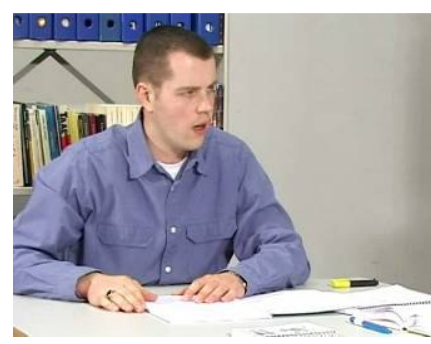

MS

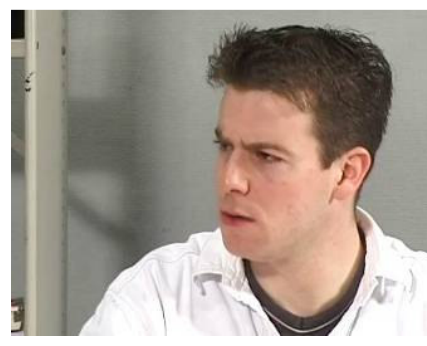

$\mathrm{CU}$

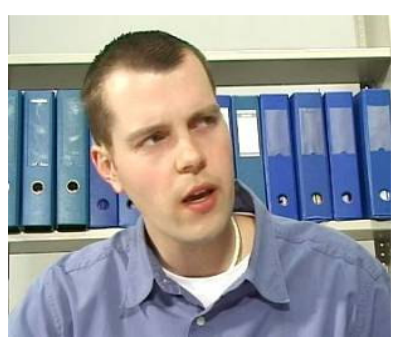

$\mathrm{LCU}$

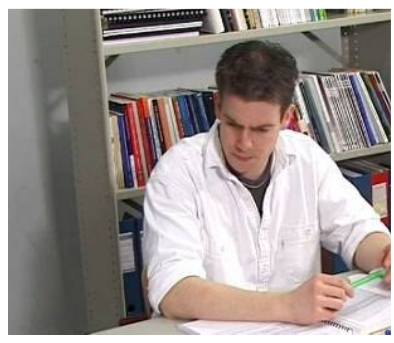

H MS

Figure 5.2: Representative shots of the three web trigger video designs

Perceived emotional valence response. To avoid participants being aware of their emotions and how this may colour their interpretation, an indirect measure for emotional valence response was used. As already stated in the introduction of this chapter (see 5.1), the affect-as-information theory of Schwartz (2001) proposes that people use their affective state as an information cue for the interpretation of or attitude about a given situation (i.e., a person, situation, or object): when it feels good (or bad), it is good (or bad). Therefore, instead of articulating their own emotions, participants will be asked to judge the emotional valence responses of the characters in the trigger video. This creates an indirect measure of emotional valence response and a situation in which affect, as caused by video design, may become an information cue for judging the persons and the situation they are in as presented in the trigger video. 
The Self-Assessment Manikin (SAM) was used to assess the perceived emotional valence responses of each character. SAM is a visual scale which represents an emotional response to a stimulus, with respect to the dimensions valence, arousal, and dominance (Bradley \& Lang, 1995). SAM reflects each dimension with a graphic character arrayed along a continuous 9-point scale. For valence, SAM ranges from a smiling happy figure to an unhappy figure (see also Figure 4.2 in Chapter 4).

Perceived personality of characters. The two basic dimensions of personality of media characters are dominance and friendliness (Reeves \& Nass, 1996). This is comparable to the horizontal and vertical axes or dimensions in interpersonal judgements (e.g., Argyle \& Dean, 1965; Birtchnell, 1996; see also Figure 5.1). Two items, using a 9-point semantic differential scale, scored directly for these two dimensions (i.e., dominant/submissive; friendly/unfriendly).

For exploration purposes, additional personality items were included based on Cattell's 16 personality factors. They represent the most important personality factors according to Cattell and Cattell (1995). The factors are warmth, reasoning, emotional stability, dominance, liveliness, rule-consciousness, social boldness, sensitivity, vigilance, abstractness, privateness, apprehension, openness to change, self reliance, perfectionism, and tension. Although factors that relate to dominance (e.g., self-reliance), emotionality (e.g., emotional stability), and friendliness perceptions form the main interest for the present study, all factors were examined. The factors were translated to Dutch and transformed into 16 bi-polar items using a 9-point semantic differential scale (the Dutch version is presented in Appendix D).

Perceived problem situation. This dependent measure was used to examine whether audiovisual design may also have an impact on the perception of the problem situation presented in the web trigger video (RQ4.2). A multiple choice question provided possible solutions to the problem. Since the scene portrayed a conflict situation, solutions included representatives of the five main styles in conflict handling: integrating, obliging, dominating, avoiding, and compromising (Rahim \& Magner, 1995). Also, an indication of the emotion felt by the main character was incorporated (an angry reaction or a friendly reaction, for example). This resulted in a list of 12 possible reactions for the main character from which subjects had to select the most obvious one at the climax of the conflict (see also Table 5.3). 
Additional arousal control measure. For the purpose of additional analysis, the perceived emotional arousal level of the characters was assessed as well. The arousal dimension of the Self-Assessment Manikin (SAM) was used for this (Bradley \& Lang, 1995). For arousal, SAM ranges from an excited figure to a sleepy figure (see also Figure 4.2 in Chapter 4).

\subsubsection{Procedure}

The experiment was conducted simultaneously in two university computer rooms that each comprised 20 individual computer workstations with 17-inch monitors. The experiment was carried out on one day in five rounds.

Participants were randomly assigned to one of the three conditions. An experimental session lasted approximately 25 minutes. The experimental procedure and the instruments (questionnaires) were automated into a webbased environment. Figure 5.3 shows two screens from the web-based environment.
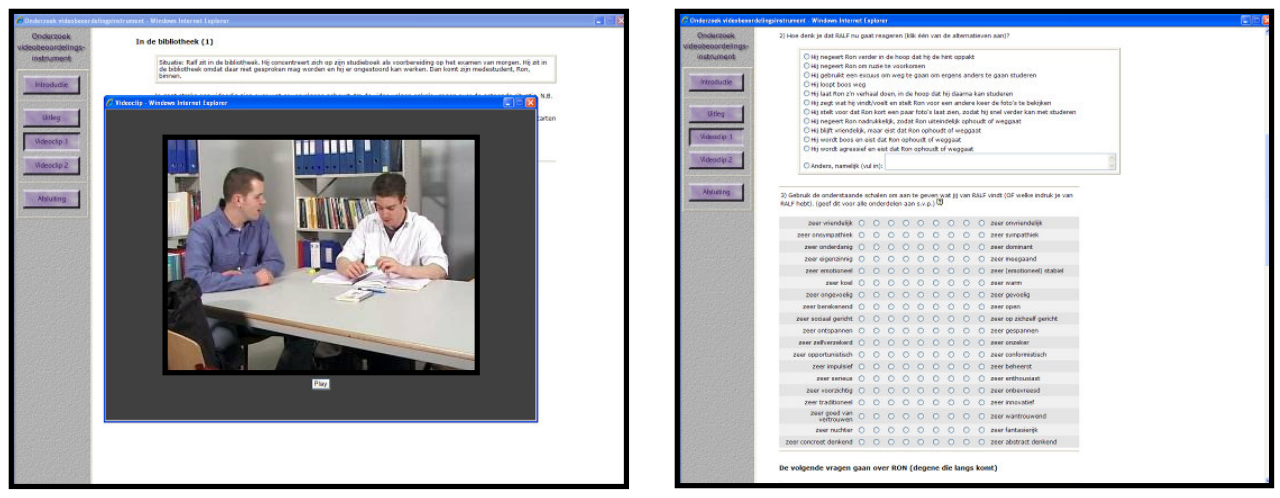

Figure 5.3: Screens from the web environment. Left: video window displaying the library problem. Right: questionnaire presented afterwards

The left side of the figure shows the video clip of the library problem displayed in a video window. The right side of the figure shows the web page with the questionnaire (see also Appendix D). This experimental web environment ran from a local drive of the computer, to be independent of web-servers and to control for technical smoothness of presentations. Data were collected online using a local area network. 
The general procedure was as follows: after a brief introduction to the experiment, participants were asked to click on the monitor desktop's icon to launch the experimental web environment. This initiated an introduction providing information on the purpose of the experiment as well as the types of questions used in the questionnaires. The experimental section was initiated by showing a web page that introduced the vignette problem as follows: "Ralph is sitting in a library. He concentrates on his study book in preparation for tomorrow's exam. He sits in the library because it is a zone of silence where he can work undisturbed. Then his fellow student, Ron, enters." Subjects were instructed to put on the headset and click on the link for opening the video display window. In this window one version of the experimental video vignette clips (neutral, close-up, or dominance) could be started immediately by clicking on the play button. After watching the video clip, a questionnaire was presented that measured the viewer's perception of the characters and the situation they were in (see Appendix D). The experiment was concluded online by thanking the participants for their participation.

\subsection{Results for the Experimental Trigger Video Designs}

For each of the hypotheses and research questions the outcomes of the experiment will be presented in the next sections.

\subsubsection{The Close-up (Proximity) Version: Impact of Camera Shot Length}

Perceived emotional valence response of main characters. Hypothesis 4.1.1 stated that, compared to the neutral version, the close-up version would have the following impact on perceptions of the emotional valence responses of the main character (shot in close-ups): the main character, who expresses a negative emotion, would be rated as more negative (as a result of the intensifying effect of close-up shots). A Mann-Whitney test showed that the main character was indeed perceived as significantly more negative ("unhappy") in the close-up version (Mean Rank $=46.80$ ) compared to the neutral version (Mean Rank = 34.76), $Z=-2.47, p=.007$, as measured by subjective reports on the SAM valence dimension. This result supported the proposed intensifying effect of close-up shots. 
Perceived personality of main character. Hypothesis 4.1.2 predicted that, compared to the neutral version, the main character would be rated more extremely on personality evaluations in the close-up version (as a result of the intensifying effect of close-up shots). Mann-Whitney tests were conducted to compare the two versions. For the two basic personality dimensionsfriendliness and dominance-no significant differences were found when the close-up version was compared with the neutral version, respectively, $Z=-.71$, ns and $Z=-.32$, ns. This hypothesis was thus not supported by the results for basic personality dimensions.

For the personality items based on Cattell, three items produced significant effects. In the close-up version the main character was rated as significantly more (emotional) reactive (Mean Rank $=34.19$ ) than in the medium shot (Mean Rank = 48.33), $Z=-2.75, p=.003$. Also, the main character was rated as significantly more wilful (Mean Rank $=35.21$ ) in the close-up version, compared to the medium shot (Mean Rank $=47.23$ ), $Z=-2.38, p=.009$. Finally, the main character was perceived as significantly less restrained (Mean Rank = 36.92) in the close-up version, compared to the medium shot (Mean Rank = 45.40), $Z=-1.67, p=.05$.

RQ4.1.1 explored whether the close-up version would result in different perceptions of emotional valence responses and the second character's personality, compared to the neutral version. This would indicate that a different audiovisual design for one character may affect the relative roles of both characters. Mann-Whitney tests were conducted for the emotional valence dimension (SAM) for the two basic personality items (friendliness and dominance) and for the 16 personality items based on Cattell.

Perceived emotional valence response of the second character. A MannWhitney test showed that the second character (in medium shot) was not perceived differently when the main character was portrayed in close-up (Mean Rank = 38.13) or, as the second character, in medium shot (Mean Rank = 44.09), $Z=-1.19$, $n s$, as measured by subjective reports on the SAM valence dimension.

Perceived personality of second character. The second character was rated as more dominant on the basic personality dimension in the close-up version (Mean Rank $=48.88)$, compared to the neutral version (Mean Rank = 32.51), 
$Z=-3.24, p=.001$. Additionally, for four out of the 16 personality items, perceptions of the second character changed significantly as a result of the close-up used for the main character. For another two items this change approached significance. Table 5.1 provides an overview of the significant effects related to perceived personality of the second character. Apparently, when showing more of the emotional expressions of the main character, the second character appears more intrusive-more dominant, reserved, utilitarian, relaxed, self-assured, lively, and uninhibited. The latter two can be expected to be negatively valenced in the situation where the main character is trying to study.

Table 5.1: Effects of main character close-ups on second character's

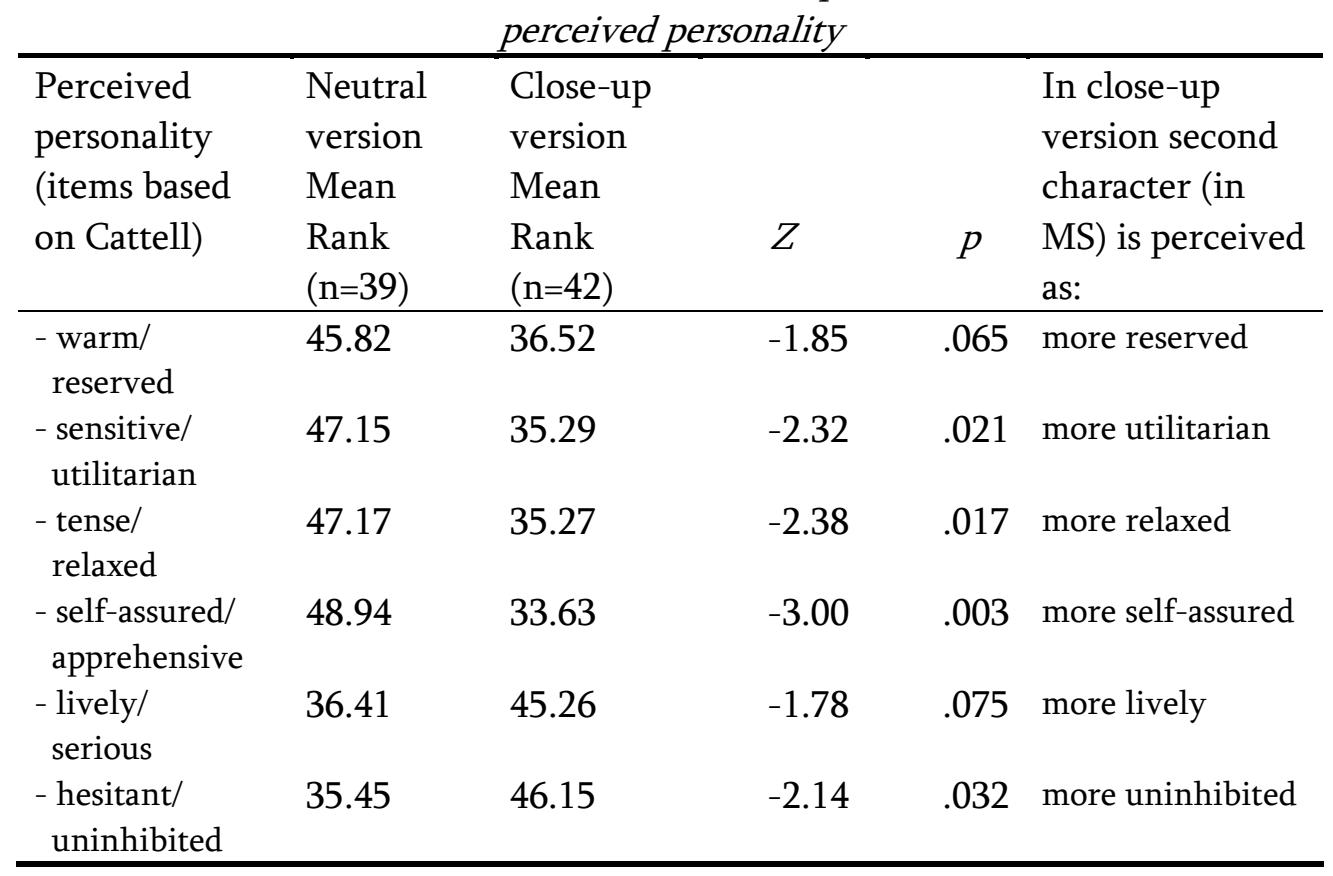

\subsubsection{The Dominance Version: Impact of Combined Camera Height and Shot Length}

Perceived emotional valence responses of characters. Hypothesis 4.1.3 stated that, compared to the neutral version, the dominance version would have the following impact on perceptions of the emotional valence responses of the main character (in high-angle medium) and the second character (in low-angle 
close-up): (a) the main character would be rated as more negative (unhappy), and (b) the second character would be rated as more positive (happy). MannWhitney tests were conducted to examine differences between the two versions.

The main character was perceived as significantly more negative ("unhappy") in the dominance version (Mean Rank $=48.29$ ) compared to the neutral version (Mean Rank = 33.15), $Z=-3.06, p=.001$, as measured by subjective reports on the SAM valence dimension. This result supported Hypothesis 4.1.3(a).

The mean rank of emotional valence responses for the second character was about the same in both the dominance and in the neutral version (respectively 40.46 and 41.58). The camera manipulation had no effect on perceptions of the second character's emotional valence responses, $Z=-.22$, $n s$. Hypothesis 4.1.3(b) was therefore not supported by the data.

Perceived personality. Hypothesis 4.1.4 predicted that, compared to the neutral version, the dominance version would have the following impact: (a) the main character (in high-angle medium) would be rated more submissive and more positive on personality evaluations, and (b) the second character (in low-angle close-up) would be rated more dominant and more negative on personality evaluations. Mann-Whitney tests were conducted to compare the versions.

For the main character, no differences were found on the basic personality dimension of dominance between the dominance version (Mean Rank $=41.10)$ and the neutral version (Mean Rank $=40.90$ ), $Z=-.04$, ns. The camera manipulations did not make the main character appear more submissive, as was hypothesised. The hypothesis also predicted more positive personality evaluations for the main character. Although the main character appeared more positive (friendly) on the basic personality dimension of friendliness in the dominant version (Mean Rank $=37.69$ ) compared to the neutral version (Mean Rank = 44.56), this difference did not reach significance, $Z=-1.35, p=.09$. Additionally, no effects were found on the personality items based on Cattell that indicated more positive evaluations of the main character.

It was expected that the second character (in low-angle close-up) would be rated more dominant and more negative on personality evaluations. MannWhitney tests were conducted to compare the versions. In the dominance version, the second character was indeed perceived as significantly more 


\section{Chapter 5}

dominant on this basic personality dimension (Mean Rank $=49.69$ ) than in the neutral version (Mean Rank = 31.64), $Z=-3.58, p=.000$. In line with the hypothesis, the second character appeared more negative (unfriendly) on the basic personality dimension of friendliness in the dominant version (Mean Rank $=44.70$ ) compared to the neutral version (Mean Rank = 37.01). This difference approached significance, $Z=-1.49, p=.07$. Additionally, Table 5.2 provides an overview of the significant effects found on several of the personality items based on Cattell. Comparing the dominance with the neutral version, the findings indicate that the second character was perceived as significantly more private, utilitarian, self-assured, lively, uninhibited, and imaginative.

Table 5.2: Effects of dominance version on second character's perceived personality

\begin{tabular}{|c|c|c|c|c|c|}
\hline $\begin{array}{l}\text { Perceived } \\
\text { personality } \\
\text { (items based on } \\
\text { Cattell) }\end{array}$ & $\begin{array}{l}\text { Neutral } \\
\text { version } \\
\text { Mean Rank } \\
(\mathrm{n}=39)\end{array}$ & $\begin{array}{l}\text { Dominance } \\
\text { version } \\
\text { Mean Rank } \\
(\mathrm{n}=42)\end{array}$ & $Z$ & $p$ & $\begin{array}{l}\text { In dominance } \\
\text { version second } \\
\text { character is } \\
\text { perceived as: }\end{array}$ \\
\hline $\begin{array}{l}\text { - forthright/ } \\
\text { private }\end{array}$ & 48.63 & 33.92 & -2.90 & .004 & more private \\
\hline $\begin{array}{c}\text { - sensitive/ } \\
\text { utilitarian }\end{array}$ & 48.94 & 33.63 & -2.98 & .003 & more utilitarian \\
\hline $\begin{array}{l}\text { - self-assured/ } \\
\text { apprehensive }\end{array}$ & 49.91 & 32.73 & -3.40 & .001 & more self-assured \\
\hline $\begin{array}{l}\text { - lively/ } \\
\text { serious }\end{array}$ & 34.38 & 47.14 & -2.57 & .010 & more lively \\
\hline $\begin{array}{l}\text { - hesitant/ } \\
\text { uninhibited }\end{array}$ & 34.50 & 47.04 & -2.49 & .013 & more uninhibited \\
\hline $\begin{array}{l}\text { - practical/ } \\
\text { imaginative }\end{array}$ & 35.97 & 45.67 & -1.94 & .052 & more imaginative \\
\hline
\end{tabular}

\subsubsection{Impact of Trigger Design on Perceived Problem Situation}

RQ4.2 explored whether trigger designs result in different perceptions of the task and lead to different subsequent actions as selected by the viewers. This would indicate that audiovisual design of a web trigger video does have an impact on the task perception and on task-related activities. Table 5.3 presents an overview of the scores for the 5 main conflict styles. (NB Data for one of the 
123 participants was excluded from the analysis, since the answer was unclear [chosen option was "Else, namely"]).

\section{Table 5.3: Selected conflict categories for the expected main characters subsequent action}

Question at the climax of the conflict:

"What is the most likely way for Ralph to respond"?

Behavioural options for Ralph presented in a selection list (between the brackets is the frequency of a selection).

Avoiding $(f=17)$ :

1. He ignores Ron in the hope that he will get the hint $(f=3)$

2. He ignores Ron to prevent a quarrel $(f=0)$

3. He uses an excuse to leave to study somewhere else $(f=8)$

4. He leaves angry $(f=6)$

Obliging $(f=2)$ :

5. He lets Ron tell his story and hopes that he will be able to study afterwards $(f=2)$

Compromising $(f=19)$ :

6. He suggests Ron show him just a few photos, so he will be able to study quickly afterwards $(f=19)$

Integrating $(f=4)$ :

7. He expresses what he finds/feels and suggests Ron to look at the photos another time $(f=4)$

Dominating $(f=80)$ :

8. He ignores Ron explicitly, to make Ron finally stop or leave (10)

9. He stays friendly, but demands Ron to stop or leave (24)

10. He becomes angry and demands Ron to stop or leave (46)

11. He becomes aggressive and demands Ron to stop or leave (0)

12. Else, namely .... (0)

Based on the conflict situation that the trigger video showed, the most likely actions taken by the main character at the video's climax were of the conflict style "dominating": "demanding, either in a friendly or in an angry way, that the second character would be quiet or leave the room". To explore if the trigger video design had an impact on the conflict style that was selected, a Pearson Chi-Square test was performed. No differences were found between the three trigger versions, Chi-Square $(8)=6.27, n s$. 


\subsubsection{Additional Analyses}

In this section additional analyses will be discussed. First, the potential impact of video design on perceived emotional arousal responses of characters is explored. Second, an additional analysis is conducted to explore whether emotional valence might have played a role in the selection of the main character's action, since these action descriptions were generally not formulated neutrally.

Audiovisual design and emotional arousal responses. Next to the video content, formal attributes of an affective video message can also elicit emotional arousal responses that may impact cognitive processing (Lang, 2000). Therefore the potential impact of video design on perceived emotional arousal responses of characters was explored (see also Chapter 2.3.2). A comparison was made between the three trigger video versions on perceived arousal responses for each character.

Table 5.4: Mean ranks of emotional arousal responses for main and second character in each trigger version

\begin{tabular}{|c|c|c|c|c|c|}
\hline \multirow{2}{*}{$\begin{array}{l}\text { Perceived } \\
\text { emotional arousal } \\
\text { response }\end{array}$} & \multicolumn{3}{|c|}{$\begin{array}{l}\text { Trigger video version } \\
\text { (Mean Rank) }\end{array}$} & \multirow{2}{*}{$\begin{array}{l}\text { Chi- } \\
\text { square } \\
\text { (df) }\end{array}$} & \multirow[b]{2}{*}{$p$} \\
\hline & $\begin{array}{c}\text { Neutral } \\
(\mathrm{n}=39)\end{array}$ & $\begin{array}{c}\text { Close-up } \\
(\mathrm{n}=42)\end{array}$ & $\begin{array}{c}\text { Dominance } \\
(\mathrm{n}=42)\end{array}$ & & \\
\hline Main character & 60.51 & 64.24 & 61.14 & $.30(2)$ & .86 \\
\hline Second character & 68.73 & 68.25 & 49.50 & $8.03(2)$ & .02 \\
\hline
\end{tabular}

Note. A lower mean rank score represents a higher arousal level.

As Table 5.4 indicates, audiovisual design did not have an impact on how the main character's emotional arousal responses were perceived. However, for the second character, an effect was found. As the table shows, the dominance version produced the lowest Mean Rank scores for the second character, meaning that this version made him appear the most aroused, as measured by subjective reports on the SAM arousal dimension. A Kruskal-Wallis test indicated that the differences between the three versions were significant, ChiSquare $(2)=8.03, p=.03$. 
Emotional valence in description of subsequent action. This analysis was conducted to explore whether affective responses, as caused by video design, may become an information cue for judging the persons and their situation (as depicted in the trigger video) in a way that affects the judgement of that situation in an affect-congruent way (e.g., Schwartz, 2001). It was expected that emotional video designs that emphasise negative emotions will result in the selection of more negatively valenced actions for the main character compared to the neutral design. To explore the potential role of emotional valence in the task content, all possible actions of the main character were categorised according to the message they would send out to the second character. The main character's actions were categorised as negative, when the action descriptions contained a negative emotion (being angry) or were likely to have negative social connotations (being demanding and ignoring the other). Similarly, positive messages were the action descriptions that contained a positive emotion (being friendly) or were likely to have positive social connotations (being accommodating, as in compromising and excusing oneself). The versions were compared for positive/negative valence in the action description (see Table 5.5).

Table 5.5: Crosstab for expected main characters subsequent action categorised by valence

\begin{tabular}{lcccc}
\hline Emotional valence of & \multicolumn{3}{c}{ Trigger video version } & Total \\
\cline { 2 - 4 } subsequent action & Neutral & close-up & dominance & \\
\hline Negative & 14 & 25 & 26 & 65 \\
Positive & 25 & 17 & 15 & 57 \\
Total & 39 & 42 & 41 & 122 \\
\hline
\end{tabular}

Note. Negative action $=1,2,4,8,10$; positive action $=3,5,6,7,9,11$; see Table 5.3

It was found that the (emotional) versions with camera manipulations (the close-up and dominance version) had higher selections of negative-valenced actions; whereas positive-valenced actions were more often selected in the neutral version. A Pearson Chi-Square test indicated that the differences were significant, Chi-Square (2) $=7.08, p=.03$. 


\subsection{Discussion}

This chapter addressed the fourth and final research question (RQ4) that was presented in Chapter 1: does audiovisual design influence viewer responses to (trigger) video content and related task activities, when both are presented in a web-based environment? RQ4.1 examined the impact on the perceived emotional valence responses and the perceived personality of two interacting characters and their relative roles in a trigger video, while RQ4.2 explored the impact on the perceived problems situation, as presented in the trigger video. For RQ4.1 four hypotheses were developed that predicted specific effects of audiovisual design for each of the two characters.

\section{Audiovisual Design of Trigger Videos}

As a useful starting point for selecting relevant production variables that may influence perceptions, we borrowed from interpersonal psychology the notion of two axes or dimensions of social interaction (e.g., Birtchnell, 1996). The horizontal distance or proximity between persons seemed closely connected to camera shot length, and the vertical distance or inequality seems comparable to camera height (or vertical angle). Based on this and the available research, predictions were made on how audiovisual designs involving those two axes would have an impact on perceptions of the characters' emotional valence response and personality. The findings for each of the audiovisual designs of the trigger video are as follows:

Close-up (proximity) version. This trigger version was created to examine the impact of camera shot length. It was compared with a version that used medium shots for the main character. For camera shot length, we found support for the intensifying effect of close-ups: for the perception of the main character's emotional valence response, a convincing intensifying effect was observed (Hypothesis 4.1.1a). For the personality perception (Hypothesis 4.1.2), there was no effect on the two basic personality items. However, the Cattellbased personality items provided some indications that the main character appeared as more (emotional) reactive, wilful, and less restrained. Although the close-ups used for the main character did not influence the perception of the second character's perceived emotional valence response, the present study found strong support for an impact on the relative roles as measured by personality evaluations (RQ4.1). Both the basic dominance dimension and several Cattell items changed significantly, when the audiovisual design for the main character was changed (e.g., the second character appears more dominant, 
reserved, utilitarian, relaxed, self-assured, lively, and uninhibited when the main character is shot in close-up).

Dominance version. Camera height and shot length were combined in the dominance version to emphasise the inequality in status between the two characters to maximize the impact on the perceptions of the two characters' relative roles in the trigger video. The results of our experiment showed that the predicted effects on perceptions of emotional valence state only occurred for the main character (supporting Hypothesis 4.1.3a); this character seemed unhappier when portrayed in high-angle medium shots. The predicted effects on personality perceptions were only present for the second character (supporting Hypothesis 4.1.4b); his dominance and "rudeness" may have been emphasised by portraying him in low-angle close-up shots. The additional analyses further showed that in this version the second character was perceived as being mostly aroused, compared to the close-up and neutral version.

\section{Trigger Videos and Task-related Activities}

For the use of trigger videos in a web-based learning environment a central question is whether these different designs also affect the perception of the scene and as such affect post-video activities differently (e.g., Cyrs, 1997), or, as RQ4.2 asked: Does audiovisual design influence the perception of the problem situation, as indicated by viewer selections of (re)solutions to the problem situation that is presented in the web trigger video? Based on the item-selection list for assessing the main character's most likely course of action, no differences were found between the trigger video versions. It must be noted, that in the present study a preset list of items was used. It might be advisable to examine this issue more thoroughly by using open-ended answer formats like fill-out forms or capturing thought processes by using thinking-out loud protocols. These methods might provide more insight into the influence of audiovisual design of triggers on the processing of subsequent activities. Also, the specific language used in provided answers might indicate a possible impact of affect (e.g., Beukeboom, 2003).

In the additional analyses it was explored whether audiovisual design did have an impact on scene interpretation, when looked at from an action-valence perspective. Conditions with camera manipulations (the close-up and dominance version) showed more negatively-valenced selected actions, whereas positively-valenced actions were more often selected in the neutral trigger condition. This might be explained by the role of affect in evaluating 
persons and situations, as was summarised in Chapter 2.3.2. The different video designs may have evoked different affective responses in viewers that may have then coloured interpretations in an affect-consistent way. In the present study, however, only perceived emotional valence responses of on-screen characters were assessed. In the future it may be advisable to also measure affective responses evoked in viewers, to examine this potential congruency effect.

In all, designers of trigger videos rightly advised the use of audiovisual design or production techniques to maximize or emphasise the emotional impact of the scene (Rushby, 1987). As the present study has shown, design not only has an impact on the emotional layer of the scene, it also impacts the perceptions of the characters and, more precisely, their relative roles in affective web-video. Even though the present study was limited to the use of one video story designed in only three different ways, it does indicate that audiovisual design influences viewer responses to (trigger) web-video content and may have an impact on related task activities, when both the video and the activities are presented in a web-based environment. Design and application of web-videos in an educational task-setting might thus affect the perception/experience of the task and, consequently, the task outcomes.

\subsection{Conclusions}

This chapter addressed the fourth research question presented in Chapter 2:

- Does audiovisual design influence viewer responses to (trigger) video content and related task activities, when both are presented in a webbased environment (RQ4)?

We investigated the first part of RQ4 with the help of RQ4.1 that focused on audiovisual design's influence on emotional valence responses and personality evaluations of characters in a web trigger video. The results affirmed this part of RQ4 for the conditions of this study. The second part of RQ4 was investigated with the help of RQ4.2 that focused on the influence of audiovisual design on the perception of the depicted problem situation. The existence of this influence could not be affirmed. However, the additional analyses showed that (negative) emotional designs resulted in more negatively-valenced selected actions, indicating an affect-congruent influence on the perception of the problem situation. 
Our findings indicate that audiovisual design can be effectively applied to intensify perceptions of emotional valence for the main character in a trigger video. Moreover, close-ups may make the main character appear stronger (e.g., more reactive and wilful). This might be explained by the potential threatening effect of close ups (Persson, 1998) and by the absence of information of bodily tension that might indicate uneasiness (Argyle, 1967). Following Kraft (1987), the present study examined the impact of camera manipulation on the perception of two characters in a story. A remarkable effect was found for the impact of shot length applied for the main character on personality evaluations of the second character: their relative roles were strongly accentuated. This finding is in line with and extends the study of Kraft, who examined the relative roles of characters in picture stories.

Note:

A version of this chapter is submitted for publication: Verleur, R., Verhagen, P. W., \& Heuvelman, A. (2008). Trigger videos on the web: Impact of audiovisual design. Manuscript submitted for publication. 


\section{General Discussion}

\section{Presentation and Task Effects of Affective Video on the Web}

The purpose of the present PhD study was to explore whether and how video applied in a web environment can be effective in evoking affective responses and how these responses may influence task performance. Four research questions were developed and explored in three experiments. This chapter presents an overview of the findings of this PhD project, the conclusions that can be drawn from them and how these findings contribute to the main question of the study. The chapter concludes with a discussion of the PhD project, by addressing its limitations, discussing the conclusions, implications for educational practice, and providing suggestions for continued research on presentation and task effects of affective video on the web.

\subsection{Summary of Findings}

This $\mathrm{PhD}$ project started off with the following main question for the study: "How do affective responses to video influence task performance in a web environment?" Affective response is defined as a positive or negative valenced emotional response or mood state. Chapter 2 developed a theoretical framework for the underlying relationships between video and affective responses and between affective responses and performance in a task setting. In addition, the potential impact of the web environment as medium context on the affective potential of video in a task setting was explored. The framework was used to develop four research questions that were studied in three experiments. The main components of the project, being video, affective responses, task setting, and the web context were presented in a conceptual model (see Figure 6.1) that 
visualises the relationships between the variables that are examined in each of the three experiments (labelled Study 1, Study 2, and Study 3). The findings for each of the studies indicated in the figure will be presented in subsequent sections.

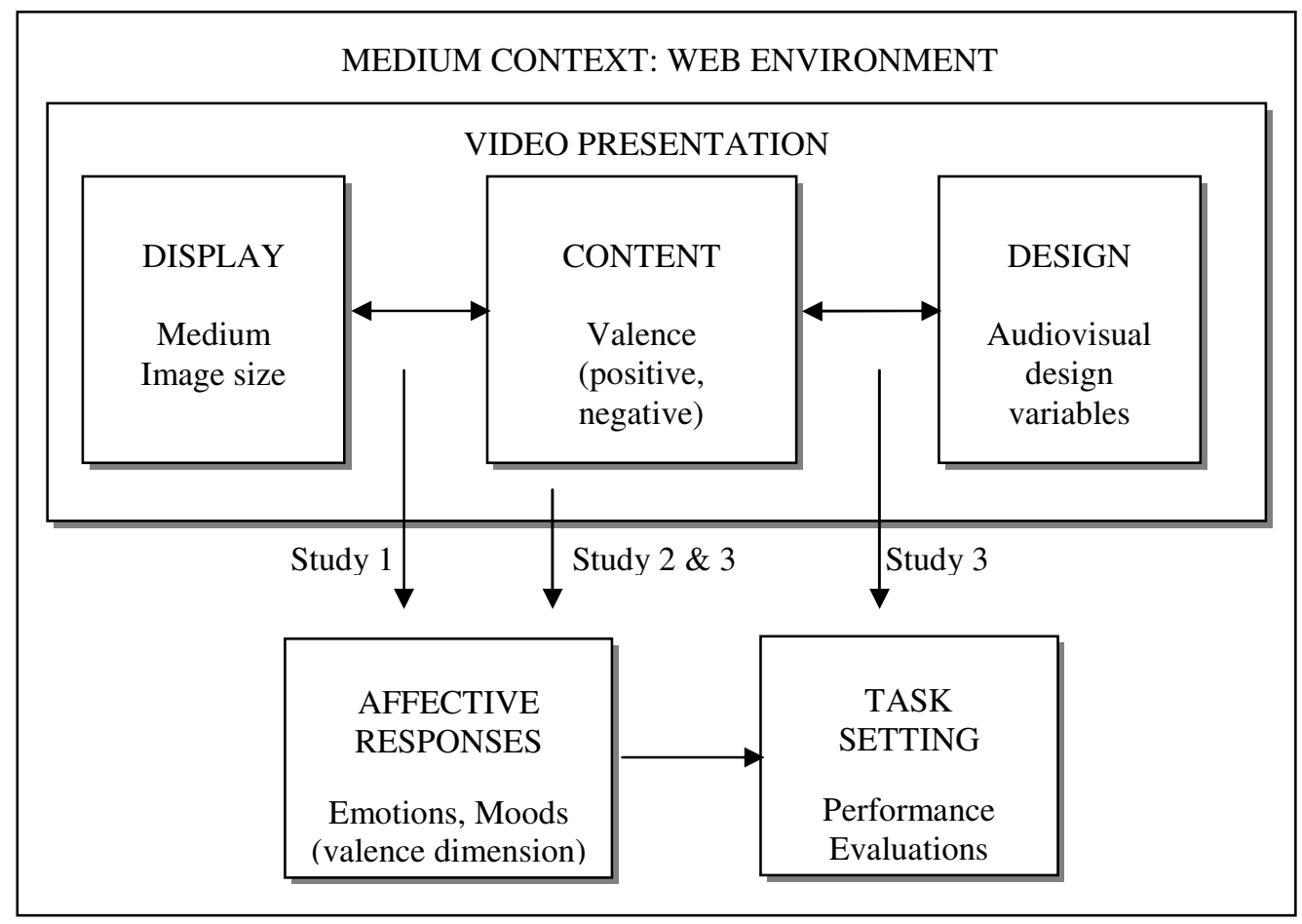

Figure 6.1: The conceptual model of the PhD project: Video Presentation, Affective Responses, and Educational Tasks

\subsubsection{Presenting Affective Video on the Web}

In Chapter 3, the issue of video presentation in a web environment was explored. A study titled "Affective responses to video: Do medium and image size matter?" was presented about the relationship between affective video content and video presentation. In Figure 6.1 this study is indicated by the arrow labelled 'Study 1'. In this study valence of video content (positive versus negative), medium (web versus television), and image size (small versus large) are the independent variables. Affective responses are the dependent variables. The study addresses two research questions: RQ1 and RQ2. 
RQ1 explored whether positive and negative video materials produced similar affective responses when applied in a web-based environment as compared to a television setting. Although our introduction suggested that different media may lead to different perceptions and experiences, our findings indicated that this was not the case for the affective responses we measured. The two medium settings were equally effective in evoking emotional valence responses and inducing mood changes in participants.

RQ2 examined the effect of image size on affective responses to positive and negative video content. This question was broken down into two more specific questions. The first (RQ2.1) examined a potential intensifying or accentuating effect of image size on emotional valence responses. The second (RQ2.2) examined this effect on induced mood changes. Although some of the literature suggested that emotional valence responses might not be affected by image size manipulations, this study did find an image-size effect for video content that varied on a valence dimension. Our findings for combined medium settings showed that larger images produced significantly more intense emotional valence responses and mood changes for affective video content. Also in the web setting, this effect was clearly demonstrated for the positive video clip (a "situation comedy").

\subsubsection{Impact of Video-evoked Affective Responses on Unrelated Tasks: The Case of Problem Tasks}

In Chapter 4 a study titled "Can mood-inducing videos affect unrelated (problem-solving) activities in a web-based environment?" was presented. In Figure 6.2 this study is indicated with the arrow labelled 'Study 2'. In this study video content (positive versus negative) is manipulated in an experimental setting to induce a (positive or negative) mood state in participants before they have to carry out problem-solving tasks that are not related to the content of the video. The performance on these problem-solving tasks is the dependent variable.

Based on Kaufmann and Vosburg's studies (Kaufmann \& Vosburg, 1997;

Vosburg, 1998), we expected that problem-solving activities in an optimising task condition (i.e., the problem is solved when the solution matches the objective solution criteria) would be impacted by a negative mood, and that 
problem-solving activities in a satisficing task condition (i.e., the problem is solved when the user is satisfied about the given solution[s]) would be impacted by a positive mood.

Our findings supported the expectations for an optimising task: viewing a negative video clip improved performance while exposure to a positive video clip reduced performance on solving the insight problem (the two-string problem). The expectation for a satisficing condition was not supported: the positive video clip did not affect performance on the divergent-thinking task (the class problem).

In all, this study provided evidence that mood-inducing video clips may affect subsequent unrelated problem-solving activities, when both are presented in a web-based environment (RQ3).

\subsubsection{Impact of Video-evoked Affective Responses on Related Tasks: The Case of Trigger Videos}

Finally, in Chapter 5 a study was presented in which the focus on task context is continued and extended by examining the role of video design, this time with video content as an integral part of the content of the task. In the model, this study is indicated by arrows labelled 'Study 3', see Figure 6.2. The study titled "What is the impact of web trigger video design on emotional responses and related task activities?" addresses whether audiovisual design influences viewer responses to (trigger) video content and related task activities, when both are presented in a web-based environment (RQ4). The study examined whether audiovisual design influences emotional responses (i.e., perceptions of emotional valence responses) and personality evaluations of characters to a trigger video, when both are presented in a web environment. For that purpose a trigger video was used in which a conflict situation develops between two characters.

The findings showed that audiovisual design can be effectively applied to intensify perceptions of emotional valence for the main character in the trigger video. Moreover, close-ups may make the main character appear stronger (e.g., more reactive and wilful). A striking effect was found for the impact of shot length applied for the main character (portrayed in close-up) on personality 
evaluations of the second character (portrayed in medium shot): their relative roles were strongly accentuated. This finding is in line with and extends the study of Kraft (1987), who examined the relative roles of characters in picture stories. The study did not find an impact of audiovisual design on perceived problem situation as indicated by viewer selections of (re)solutions to the problem situation presented in the web trigger video.

\subsection{Discussion}

In Chapter 1 it was stated that "The present project can be placed at the intersection where educational technology, media psychology, video design, and experimental research on affect and cognition coincide." As a result the study used a multi-perspective theoretical approach. The findings of the study will be discussed in their respective theoretical context.

\subsubsection{Potential of Affective Video on the Web}

The first finding of this study is that affective video appears to be effectivecomparably so to television viewing - in evoking affective responses to video when it is presented in a web-environment (RQ1). So, the web is equally effective in evoking emotional valence and mood state changes in viewers, as in a television setting.

This finding first affirms video's capability to evoke affective responses, which is (as indicated in Chapter 1) generally acknowledged in educational practice (e.g., Romiszowski, 1988). Koumi (2006) contends that the rich symbol system of video provides substantial realism, which is exploited to create enduring affective influences of motivations and feelings. Koumi calls this category of added value "the nurturing value of video". This potential of video to evoke affective responses follows earlier research studies (see Chapter 2) on emotion and mood induction (Gerrards-Hesse, Spies, \& Hesse, 1994; Gross \& Levenson, 1995; Philippot 1993; Rottenberg, Ray, \& Gross, 2007) and on psychological responses to television content (Reeves \& Nass, 1996). Moreover, the present study extends this affective potential of video to affective video presented in a web environment. Our finding also agrees with recent studies addressing contemporary media. They suggest that video presented by modern devices, 
such as mobile phones, small LCD displays, and iPods may also evoke emotional responses (e.g., Ravaja, 2004).

The finding that affective responses to television and web-presented video were similar for both medium settings was not self-evident. The literature suggested that these media might be perceived differently by users (Ferguson \& Perse, 2000; Nielsen, 1997, 2005). Nevertheless, there are possible explanations why a differential effect on affective responses did not occur in our study. First, the experimental setting might have changed the overall perception. For example, the awareness of participating in an experiment, the group of participants (students) that were enrolled in the experiment, who might use computers for relaxation and to kill time, and the media employed in the study might have been different from the typical media distinctions and media users as described or intended by Nielsen.

It could be argued that, although media perceptions might have been different, these were ruled out by the (affective) video content, which was the same. Media comparison studies have been conducted in an educational context, since media are employed in education and especially when new media are entered in educational practice. In general, these studies show a positive effect for the new medium that is being compared to a more traditional medium. However, this effect is commonly discarded as a "novelty effect" (e.g., Clark, 1983; Liao \& Bright, 1991). The general conclusion from educational media comparison studies is that there are no significant differences. Since web-video use was not novel at the time of the study, it was not expected to find a novelty effect. Moreover, we found a no-significant-difference effect (see also discussion in Koumi, 2006). As suggested by authors such as Kozma (1994), the media (the vehicles in terms of Clark, 1983) themselves should not be compared, but their capabilities or attributes. The most prevalent attribute was, in our case, the affective video content. Since this attribute was similar in both conditions, this could explain the no-significant differences we found when comparing media.

This might also be a likely explanation given the evidence that Reeves and Nass (1996) collected on the automatic (unconscious) responses to media and media content, as if they are real people, places, or situations. This implies that the media (the vehicles) themselves are of less importance. This would not only explain why video is also capable of evoking affective responses in a web setting, but also that the presentation medium is subsidiary. Even when viewers may have had different perceptions of the media, they may have responded 
primarily to the affective content or attributes, and as the affective video content was the same, so were the evoked affective responses.

In all, the present study shows the strong, affective potential of video, which persists under different media conditions. This might imply that the affective potential of video is mainly determined by the (audiovisual) attributes of video, and not by the medium.

\subsubsection{Presenting Affective Video on the Web}

The present study demonstrated that the way video is presented on the web matters. More specifically, the image size (RQ2) and the audiovisual design (RQ4) impact affective responses to video content.

The study examined the impact of image size on affective responses to video (RQ2). The findings indicated that image size influenced affective responses; thus we found an effect for emotional valence responses and mood state changes. Effects on these measures are an extension of the existing literature on image size effects. An oft cited study by Detenber en Reeves (1996; "The bioinformational theory of emotion....") strongly suggested that emotional valence responses are not sensitive to image size manipulation. Similarly, Grabe, Lombard, Reich, Bracken, and Ditton (1999) suggested that more psychological content like, for example, drama would not be affected by the presentation attribute of image size. Our study clearly found a different result: both emotional valence responses and mood state changes were affected by image size. A larger image size accentuated the affective responses.

Findings also indicated that the presentation attribute audiovisual design matters as well. This was explored in the third experiment for a trigger video (RQ4), as a useful and practical example of an affective video that explicitly aims at evoking affective responses in the viewer, and that is presented as part of a (production) task. Moreover, for trigger videos it is advised to exploit audiovisual design to emphasise the emotional impact of the scene (Rushby, 1987). In this experiment we examined the impact of camera manipulations (camera shot length and camera height) that might alter the viewer responses to and perceptions of the interpersonal relationship of the two characters in the scene (Birtchnell, 1996). The resulting "emotional designs" (i.e., a close-up [proximity] version and a dominance version) were compared with a neutral 
version of the scene. The evoked affective responses in the viewer were not assessed, as was the case in the first experiment, but how the viewer perceived the evoked emotional valence responses in the characters of the video clip. By doing so, the aim of the experiment was less obvious to the participants and, at the same time, a possible impact on the interpretation and evaluation of the scene could be measured (this will be discussed in 6.2.3). The outcomes of this study demonstrated the potential of audiovisual design: emotional design had an impact on the perceived evoked emotional valence responses in the main character: both the close-up (proximity) version and the dominance version made the main character appear to show a more "negatively valenced" emotional response, compared to the neutral version. Thus, emotional design was effective in accentuating his negative emotional valence state.

In all, it appears that presentational attributes, such as image size and audiovisual design are well capable of accentuating or intensifying the affective tone of a video clip as presented in a web environment. Moreover, both Study 1 and Study 3 support the notion that "size matters" (Reeves \& Nass, 1996). Both the manipulations of "physical size" by image size (smaller or larger screens) and shot size (closer or wider shot lengths) and the manipulation of "psychological size" by combinations of camera height and shot length impact not only the affective responses but also personality evaluations. According to Musch and Klauer (2003), evaluation shares with emotion and mood that they are all grounded in the basic psychological valence dimension. This could imply that "size" has an overall accentuation/intensifying impact on the valence dimension of viewer responses, for emotion, mood, and evaluation.

\subsubsection{Affective Video and Tasks on the Web}

In Section 2.3.1 we argued that affective video could influence subsequent (unrelated and related) tasks. Based on the literature we contended that a video-evoked positive mood will lead to mood-congruent evaluation (i.e., selective processing of pleasant information), to assimilation, and to more flexibility and creativity compared to being in a negative mood, and that a video-evoked negative mood will lead to mood-congruent evaluation (i.e., selective processing of unpleasant information), to accommodation, and to more systematically information processing compared to being in a positive mood (e.g., Fiedler, 2001; Martin \& Clore, 2001). 
In the first experiment (Chapter 3) we explored the potential impact of videoevoked affect on a subsequent activity, as part of the additional analyses. In this study, the evoked affective responses to a video clip were measured, both in a television and in a web setting and in a large and small image-size format. After a positive or negative affective video clip, the evoked affective responses to a neutral clip were assessed. In our study we did not find a mood-congruent effect but an opposite or contrast effect. after a positive video clip the subsequent neutral clip was experienced as negative, and after a negative video clip the subsequent neutral clip was experienced as positive. This contrast effect might be explained by either the awareness of being in the role of judge/evaluator or the awareness of the evoked feelings. In the case of a judgment task, people tend to make a relative judgment: they will compare the actual video clip with the preceding video clip, which serves as a standard for reference (see Bolhuis, Heuvelman, \& Verleur, 2008, for a similar contrast effect).

In the second experiment (Chapter 4), we explicitly examined the impact of video-evoked mood on task performance in a web environment (RQ3). In accordance with the predictions of the satisficing-optimizing model (Kaufmann \& Vosburg, 1997; Vosburg, 1998), it was demonstrated that a video-evoked negative mood would facilitate an insight task that demands a best solution based on objective criteria. This outcome seems to agree with what other authors predicted, for example that a negative mood promotes accommodation and systematic processing (see Fiedler, 2001; Martin \& Clore, 2001). The prediction that a positive mood would facilitate a divergent thinking task calling for subjective (satisficing strategy) criteria could not be affirmed. As discussed in Chapter 4, the task that we used might not have been sufficiently discriminating to test this effect by itself. Nevertheless, the experiment demonstrated that video-evoked affective responses may indeed influence the outcomes of a subsequent task in a web environment, and that this effect is task dependent (task-dependent effect). The results further suggest that earlier effects from classical psychological experiments using different presentation media, such as television and print, may be transferred to a web environment, in which video and tasks are integrated.

In the third experiment (Chapter 5) the impact of emotion, as caused by "emotional" video design, was examined on related task activities (RQ4). As mentioned earlier, participants were asked to judge the emotional valence responses of the characters in the trigger video. This created an indirect 
measure of emotional valence response and a situation in which the impact of emotion, as caused by video design, could become an information cue for judging the persons and the situation they are in as presented in the trigger video. Both emotional designs (the close-up and dominance version) resulted in perceptions of more negative emotional valence responses in the main character compared to the neutral design. This indicated that audiovisual design is effective in altering viewer responses on an affective dimension. After that, the participants had to perform two content-related tasks: to judge the personalities of the characters and to interpret the trigger video scene in terms of the expected subsequent action taken by the main character. In short, the results showed that audiovisual design seems to accentuate some personality ratings, especially perceptions of the second character that had a negative (disturbing) role in the scene. Emotional design did not impact the selected subsequent action of the main character. However, when in the additional analyses the versions were compared for positive/negative valence in the action description, it was found that the emotional design versions (the close-up and dominance version) had higher selections of negative-valenced actions, whereas positivevalenced actions were more often selected in the neutral version. Overall, the emotional designs appeared to influence the interpretation of the scene, generally in an affect-congruent way: a more negative (emotional) designed clip resulted in a more negative interpretation of a person or the situation in the trigger video. This conforms to main findings from mood and cognition research (Martin \& Clore, 2001). The results also suggest that audiovisual design of web video might unconsciously influence one's affective state and act as an information cue for the interpretation of the video content (Schwartz, 2001) and thus influence related task activities.

Taken together, when we examine all three studies, we can conclude that video-evoked affective responses impact task performance, but the way do so differs. Study 3 provided some support for the general research finding that affective responses might colour evaluations of persons and a (problem) situation in an affect-congruent way (but recall that we used an indirect measure). In Study 1 a reverse, or contrast effect, was found: a subsequent neutral video clip evoked more positive responses after a negative video, and more negative responses after a positive video. These findings indicate that the impact of video-evoked affective responses is not a straightforward cause-andeffect (or "bullet" effect). As suggested in the mood and cognition literature, effects of affect on task performance may be modified or mediated by different factors. Factors may include the valence of the induced affective responses, the 
attribution of the source of the affective responses, the valence of the materials to be processed or judged, the processing goals of the person, the difficulty of the task, familiarity with the task, the interestingness of the task, whether feedback on the task performance was provided, and the motivation or incentive provided to the participants (Isen, 2002; Martin \& Clore, 2001). That task demands or processing goals play a role was supported by the results of Study 2. A task-dependent effect for video-induced mood was established for one of the tasks: a negative mood was more beneficial for the insight task than a positive mood. The discrepancy in effect between Study 1 and 2 may also have to do with the task type and with the attribution of the affective source. In Study 1 the affective source was made explicit, since the task was introduced as being about a new video evaluation instrument and to use it for indicating cognitive and affective responses to video (cf. the motivation or incentive provided to the participants). Participants were therefore aware that affect played a role in the study, that video was the source of it, and that they were in the role of judge. To prevent feelings evoked in them by the preceding video clip from biasing their judgment of the next clip, participants may even have made an overcorrection (Berkowitz, Jaffee, Jo, \& Troccoli, 2000). In Study 3 participants were not aware of the potential role of affect, since they were evaluating a (trigger) video scene and the characters in it, and were not asked to articulate their own affective responses. Eventual evoked affective responses caused by the audiovisual design might therefore have been attributed to the content of the scene (problem situation and characters). In all, the salience of affect, the awareness of the source of it, and the task characteristics might have played a moderating role on how affective responses impacted task performance. We will extend this issue in 6.4 where we will draw conclusions and provide suggestions for further research.

\subsection{Limitations of the Study}

For each of the experiments we discussed the limitations in the respective chapters. Here we will address and discuss the overall limitations of the $\mathrm{PhD}$ study. Most importantly, our study was focused on affective responses and task performance in a web-based environment. Our study was inspired by earlier work in experimental psychology on the impact of affect on task performance. This has resulted in decisions that excluded other directions. Because of the explorative nature of our study, we decided to examine a few variables in detail, in experimental studies. This makes the examination of cause-and-effect 
possible. However, this always leads to a limitation of the variables that can be examined. This means that other, potentially interesting variables were not selected and thus excluded from examination. The web environment that we developed aimed at providing the look-and-feel of a "real" web site. Normally, however, web sites would be more open and interactive and may be designed in a more sophisticated way.

We defined affective response as a positive or negative valenced emotional response or mood state. This was in line with the earlier studies that focused on the valence dimension of affect, but it excludes the arousal dimension of emotion. Arousal is often associated with attention processes and with memory performance. A recent study (Bolhuis, Heuvelman, \& Verleur, 2008), for example, found that compared to a high arousal level, a medium arousal level of an affective video resulted in better memory of subsequent video content, like a commercial, for example. This accords well with the inverted relationship between arousal and attention, which is shaped as an inverted U. At medium arousal level attention and therefore performance is optimal. In both low and high arousal conditions attention and performance is at its worst. In our study we did control for arousal responses, and the affective video clips used in experiment 1 and 2 were comparable on (their medium) arousal levels. In future studies arousal could be added, so that the factor arousal and a potential valence arousal interaction could be examined as well. Also, we did not examine the specific emotions that were evoked by the affective video clips.

Our study was inspired by mood and cognition experiments. In these experiments often one affective video segment is used to induce a certain affective state in viewers. In line with these experimental studies and the explorative nature of our study, we also used a limited amount of videos in our studies. Although the video clips we used were suitable in creating the valence responses we sought, as indicated by pre-tests, they might have varied on a number of other attributes as well. It could be argued that a broader range of videos (in both genre, valence strength, and other video attributes) would have created more message variance and would have reduced the chance of systematic between-video differences, thus enhancing the external validity of the findings (Reeves \& Geiger, 1994).

The tasks that we selected for the second experiment were production tasks (not reproduction tasks, see Fiedler, 1991) and came from experimental psychology studies. Although they might not seem typical educational tasks at 
first glance, it should be noted that problem solving is one of the higher order intellectual skills in education. The tasks that were used were limited in number and type, and only one of the two tasks (the insight task) showed the hypothesised effect.

The present study invited university students (usually within a certain age range), as participants in the experiments. Students are, however, not a representation of all people. This might have biased the outcomes of the studies. For example, students might be more occupied with computers and the Internet, and have different use patterns with it, than other people. Also, the majority of the students were women. Literature suggest that gender may play a role when it comes to responding to video content (e.g., genre) and presentational attributes such as image size or video design (Lombard, Reich, Grabe, Bracken, \& Ditton, 2000). In our study we balanced conditions for gender.

We used subjective scales to measure affective responses in our study. For the measurement of emotional valence responses physiological measures could also be used. However, this would have revealed the real purpose of the experiments to the participants that was now disguised by the research protocol used. It would have also triggered awareness of being in a laboratory setting, instead of in a setting such as an educational computer lab at the university.

\subsection{Conclusions, Implications, and Future Directions}

The main question of the study was: How do affective responses to video influence task performance in a web environment? The project made clear that (a) video in a web environment is capable of evoking affective responses, (b) the video-evoked affective responses did impact on task performance, and (c) the web environment itself appeared not to have an impact on the affective responses to video, but presentation variables, such as video image size and video design can impact the affective potential of video and thus the relationship between video-evoked affective responses and task performance in a web environment.

With respect to the potential of video to evoke affective responses, the project indicated that video has a strong affective potential that in different media settings, such as television and web-based settings, can be equally effective. The 
affective potential of video appears to be mainly determined by the audiovisual attributes of video (e.g., Kozma, 1994) or symbol systems (Salomon, 1979), and not by the medium. The medium might be considered as merely a vehicle in terms of Clark (1983). For web applications this implies that the Internet is a good stage to facilitate and exploit the affective potential of video. Educational practice tends to follow the media trends: as in everyday life, video screens will become both larger and smaller. The findings of this study suggest that video will also have affective potential when smaller screens are used. This looks promising for educational applications that typically use smaller screens, such as iPod-like applications, mobile phones, or mini TV's. Our study, however, suggests that when the aim is to evoke affective responses, such as defined in our study, then larger screens may do a better job than smaller screens. New video displays may, however, include new video properties (e.g., an auto stereoscopic multiview 3-D display, cf. de Boer, Verleur, Heuvelman, \& Heynderickx, 2008; ambilight, Philips Design - Technologies, 2008), and new use patterns (e.g., ambient intelligence, Ben Allouch, 2008). New media applications provide media settings that go far beyond the comparison of TV and web that Nielsen made in 1997 (Nielsen, 1997) or that we conducted in our project; they may affect the viewing experience. Research is needed to examine how these new video properties and use patterns may impact the affective potential of video applied in an educational context.

Next to this, the project indicated that video presentation, more specifically size, matters. Presentation variables of video, such as image size, camera shot length, and height, that physically or psychological "enlarge" the video content, might have an accentuating or intensifying impact on affective responses and evaluations to this content. This accentuating quality is, for example, beneficial for the type of affective video that we addressed in our third study: the trigger video. It should be noted that in our project we could only address a limited amount of form variables. For example, with respect to trigger videos, Rushby (1987) mentioned subjective camera techniques as an effective production technique to evoke affective responses in viewers. Research is needed to gain insight into how this and other form attributes relate to affective responses in viewers. In addition, the impacts of form aspects that relate to medium context are relevant to examine. Future studies could extend the present study by addressing the question how the immediate context of the video, such as the web application and the web page design, in combination with image size and audiovisual design might impact on how video is experienced. In examining this, principles of multimedia learning (Mayer, 2001), that are based on dual 
processing theory and the notion of a (limited) cognitive capacity, can be taken as a starting point. As long as there are limited research-based guidelines available to guide educational designers, designers should make informed decisions on the basis of research results as in our study together with guidelines from practice and their own experience. Careful formative evaluations may then lead to effective use of affective video components in educational settings.

A central issue in the project was task performance. The project indicated in all three studies that task performance will most probably be influenced by videoevoked affective responses. We provided evidence that multimedia content, more specifically video, may evoke affective responses in learners and that these affective responses (i.e., emotional valence responses and mood state) might affect tasks activities (problem solving and performance) in a web environment. Designers and instructors may not always be aware of the affective responses to those video clips, and the potential consequences in a task environment. It may seem attractive to add positively valenced video materials to a web-based learning environment, simply because this may create a positive mood in learners. However, as demonstrated in the study, such a positive mood state may not be beneficial for all types of tasks. Mood effects are task dependent. For example, the insight problem-solving task that we used showed that task performance in the web environment was inhibited by a video-evoked positive mood. It is therefore critical to be aware of and to continue examining in more detail the impact of video-evoked affect on different task types as one of the issues that researchers as well as designers should work on.

For practical applications it is essential that educational practitioners take into consideration the facilitating or inhibiting effects of mood. An instructional designer might consider restricting the openness of the web-based learning environment for certain tasks. Alternatively, it may be useful to raise learners' awareness of the intrinsic effects that might impact their performance. How this affects learning in practice would be a challenging issue for further exploration in real online learning settings. At this point it is, however, not yet possible to predict the exact effect of certain video-affective responses on task performance. We have found different effects (contrast, congruence, and taskdependent effects). Based on the theory and the particular settings of our experiments, we suggest that the moderating effects of learner and tasks characteristics should be researched in more detail. In this research the source of attribution of affective responses should be included. 
The present study supported the notion that not only "cognitive components" of the web-based learning environment can be designed to enhance learning effectiveness, but also "affective components" could be seen as strategies that an educational designer could apply for the benefit of the learning process. However, when it comes to purposefully influencing the feelings of others, especially when done implicitly, this immediately raises the ethical question as to whether "affective strategies" should be allowed for use in education. As indicated in Chapter 1, in our world "outside school" affective influences are apparent in our everyday life. We indicated the overall trend that video messages become both more emotional in content and in their packaging. This resulted, for example, in new television formats, such as reality TV and emotion TV, but also news broadcasts (commonly associated with objective information gathering and delivery) that have become more emotional in content and design as well. Purposefully influencing the affective feelings of others is most explicitly present in commercial and political advertisements. The main purpose of these forms of communication is persuasion-to persuade people to buy a product or service, or to adopt a certain point of view. It seems that many things are allowed to reach these goals in commercial and political communication. Education is to be considered as a goal-directed form of communication also, i.e., to teach people. What means are allowed in education to reach that end? May we "sell instruction like tubes of toothpaste"? The key to answering this question lies in the fact that emotional video production techniques (when applied well or "invisible") do not impact on conscious, but unconscious or automatic processing (Bargh \& Chartrand, 1999). These processes are present in all our communications in which affect plays a role, including educational settings. As is the case with all the materials we select and design for educational practice, we have an ethical and social responsibility in what and how we teach. When it comes to exploiting the affective potential of video in a web-based learning environment we have to be aware of the effects of emotional processes on learning. We hope our research has contributed to the awareness of and insight into these effects. 


\section{References}

Allen, N. (1972). The vertical dimension in Thulung classification. Anthropological Society of Oxford, 3, 81-94.

Argyle, M., \& Dean, J. (1965). Eye contact, distance, and affiliation. Sociometry, 28, 289-304.

Argyle, M. (1967). The psychology of interpersonal behaviour. London: Penguin Books.

Baggaley, J. P., \& Duck, S. W. (1976): The Dynamics of television. London: Saxon-House.

Baggaley, J. P. (1980). Psychology of the TV image. New York: Praeger.

Bargh, J. A., \& Chartrand, T. L. (1999). The unbearable automaticity of being. American Psychologist, 54 (7), 462-479.

Basil, M., Schooler. C., \& Reeves, B. (1991). Positive and negative political advertising: Effectiveness of ads and perceptions of candidates. In F. Biocca (Ed.), Television and political advertising. Volume 1: Psychological Processes (pp. 245-262). Hillsdale: Lawrence Erlbaum.

Ben Allouch, S. (2008). The design and anticipated adoption of ambient intelligence in the home. Doctoral dissertation, University of Twente, The Netherlands.

Berkowitz, L., Jaffee, S., Jo, E., \& Troccoli, B. T. (2000). On the correction of feeling-induced judgmental biases. In J. P. Forgas (Ed.), Feeling and thinking. The role of affect in social cognition: Studies in emotion and social interaction (Second Series, pp. 131-152). New York: Cambridge University Press.

Beukeboom, C. (2003). How mood turns on language. Doctoral dissertation. Free University of Amsterdam.

Birtchnell. J. (1996). How humans relate: A new interpersonal theory. Hove (East Sussex): Psychology Press.

Boer, C. N. de, Verleur, R., Heuvelman, A., \& Heynderickx, I. (2008). Added value of an autostereoscopic multiview 3-D display for advertising in a public environment. Manuscript submitted for publication. 
Bolhuis, W., Heuvelman, A., \& Verleur, R. (2008). Commercial breaks and ongoing emotions: effects of program arousal and valence on emotions, memory, and evaluation of commercials. Manuscript submitted for publication.

Borchers, T. A. (2005). Persuasion in the media age. New York: McGraw-Hill. Boud, D., \& Pearson, M. (1979, February). The trigger film: A stimulus for affective learning. Programmed Learning and Educational Technology, $16,52-56$.

Bracken, C. C., \& Atkin, D. (2004). How screen size affects perception of television: A survey of presence-evoking technology in our living rooms. Visual Communication Quarterly, 59(4), 23-28.

Bracken, C. C. (2005). Presence and image quality: The case of high definition television. Media Psychology, 7(2), 191-205.

Bracken, C. C. (2006). Perceived source credibility of local television news: The impact of image quality and presence. Journal of Broadcasting \& Electronic Media, 50 (4), 723-741.

Bracken, C. C., \& Pettey, G. (n.d.). Sounding out small screens and presence; the impact of screen size, pace \& sound. Retrieved June 1, 2008, from: http://computerwranglers.com/Com\%20589/Small\%20Screen\%20Studie s\%20ICA_11_1_CB.01.doc.

Bradley, M. M., Greenwald, M. K., Petry, M. C., \& Lang, P. J. (1992).

Remembering pictures: Pleasure and arousal in memory. Journal of Experimental Psychology: Learning, Memory, and Cognition, 18, 379390.

Bradley, M. M., \& Lang, P. J. (1995). Measuring emotion: the self-assessment manikin and the semantic differential. Journal of Behavior Therapy and Experimental Psychiatry, 25, 49-59.

Bretz, R. (1971). The selection of appropriate communication media for instruction: A guide for designers of air force technical training programs. Santa Monica, CA: Rand Corporation.

Briggs, L. J., \& Wager, W. W. (1981). Handbook of procedures for the design of instruction. Second Edition. Englewood Cliffs, NJ: Educational Technology Publications.

Bryant, J., \& Zillmann, D. (1994). Using television to alleviate boredom and stress: Selective exposure as a function of induced excitational states. Journal of Broadcasting, 28(1), 1-20.

Bryman, A., \& Cramer, D. (2005). Quantitative data analysis with SPSS 12 and 13. A guide for the social scientists. New York: Routledge. 
Buunk, B. P., Kuyper, H., \& van der Zee, Y. G. (2005). Affective response to social comparison in the classroom. Basic and Applied Social Psychology, 27(3), 229-237.

Burgoon, J. K. (1978). A communication model of personal space violations: Explication and an initial test. Human Communication Research, 4, 129-142.

Burgoon, J. K., \& Burgoon, M. (2001). Expectancy theories. In W. P. Robinson \& H. Giles (Eds.), The new handbook of language and social psychology (2nd ed., pp. 79-102). Sussex, UK: Wiley.

Cattell, R. B., \& Cattell, H. E. P. (1995). Personality structure and the new fifth edition of the 16PF. Educational and Psychological Measurement, 6, 926-937.

Cennamo, K. S. (2001). Digital Video. The art and the science [CD-Rom]. Virginia: Virgina Tech.

Childs, M., Cuttle, M., \& Riley, K. (Eds.). (2007). DIVERSE: Developing innovative video resources for students everywhere. Proceedings of the $5^{\text {th }}$ International DIVERSE Conference, 5-7 July 2005, Vanderbilt University, Nashville, USA, and the $6^{\text {th }}$ International DIVERSE Conference, 5-7 July 2006, Glasgow Caledonian University, Glasgow, $U K$. Glasgow: Glasgow Caledonian University.

Chandler, D. (2000). Notes on 'The gaze'. Retrieved September 30, 2004, from www.aber.ac.uk/media/Documents/gaze/gaze05.html.

Choi, H. J., \& Johnson, S. J. (2007). The effect of problem-based video instruction on learner satisfaction, comprehension and retention in college courses. British Journal of Educational Technology, 38 (5), 885895.

Clark, R. E. (1983). Reconsidering research on learning from media. Review of Educational Research, 53(4), 445-459.

Clark, R. E. (1994). Media will never influence learning. Educational Technology Research \& Development, 42 (2), 21-29.

Clore, G. L., Wyer, R. S., Dienes, B., Gasper, K., Gohm, C., \& Isbell, L. (2001). Affective feelings as feedback: Some cognitive consequences. In L. L. Martin \& G. L. Clore (Eds.), Theories of mood and cognition (pp. 2762). Mahwah, NJ: Erlbaum.

Cobin, M. T., \& McIntyre, C. J. (1961). The development and application of a new method to test the relative effectiveness of special visual production techniques for instructional television. Urbana: Illinois University. 
Coldevin, G. O. (1976). Comparative effectiveness of TV production variables. Journal of Educational Television, 2, 85-93.

Collis, B. A., \& Peters, O. (2000). Educational applications of web-based asynchronous video. In N. Corrie, T. Chambel \& G. Davenport (Eds.), Multimedia '99(pp. 177-186). Vienna: Springer Verlag.

Coulter, K. S. (1998). The effects of affective responses to media context on advertising evaluations. Journal of Advertising, 27(4), 41-51.

Cyrs, T. E. (1997). Using trigger videos and video scenarios to stimulate higher order learning. In T. E. Cyrs (Ed.), Teaching at a distance with the merging technologies: an instructional systems approach (pp. 275-283). Las Cruces, NM: Center for Educational Development, New Mexico State University.

Detenber, B., \& Reeves, B. (1996). A bio-informational theory of emotion: Motion and image size effects on viewers. Journal of Communication, 46(3), 66-82.

Detenber, B. H., Simons, R. F., \& Reiss, J. E. (2000). The emotional significance of color in television presentations. Media Psychology, 2, 331-355.

Detenber, B. H., Simons, R. F., \& Bennett, G. G. (1998). Roll 'em!: The effects of picture motion on emotional responses. Journal of Broadcasting and Electronic Media, 42(1), 113-127.

Ekman, P. (1992). Facial expression of emotion: New findings, new questions. Psychological Science, 3, 34-38.

Ekman, P. Friesen, W. V., \& Ellsworth, P. (1972). Emotions in the human face: Guidelines for research and an integration of findings. New York: Pergamon Press.

Eliot, T. S. (1968). Four quartets. New York: Harcourt.

Elliott, K. A., \& Keppell, M. (2000). Visual triggers: Improving the effectiveness of virtual patient encounters. In R. Sims, M. O’Reilly and S. Sawkins (Eds.), Australian Society for Computers in Learning in Tertiary Education. Ascilite 2000 Conference Proceedings (pp. 275-283). Southern Cross University, NSW.

Elliott, K. A., Efron, D., Wright, M., \& Martinelli, A. (2003). Educational technologies that integrate problem based learning principles: Do these resources enhance student learning? In G. Crisp, D. Thiele, I. Scholten, S. Barker and J. Baron (Eds), Interact, integrate, impact: Proceedings of the 20th Annual Conference of the Australasian Society for Computers in Learning in Tertiary Education (pp. 170-177). Adelaide, 7-10 December 2003. 
Escalas, J. E., \& Stern, B. B. (2003). Sympathy and empathy: Emotional responses to advertising dramas. Journal of Consumer Research, 29, 566-578.

Ferguson, D. A., \& Perse, E. M. (2000). The World Wide Web as a functional alternative to television. Journal of Broadcasting \& Electronic Media, 44 (2), 155-174.

Fiedler, K. (1991). On the task, the measures, and the mood in research on affect and social cognition. In J. P. Forgas (Ed.), Emotion and social judgments (pp. 83-104). Oxford: Pergamon Press.

Fiedler, K. (2001). Affective States Trigger Processes of Assimilation and Accommodation. In L. L. Martin \& G. L. Clore (Eds), Theories of mood and cognition. A user's guidebook. Mahwah, NJ: Erlbaum.

Fong, C., \& Woodruff, E. (2003). Web-based video and frame theory in the professional development of teachers: Some implications for distance education. Distance Education, 24 (2), 195-211.

Forgas, J. P. (1991). An introductionary review. In J. P. Forgas (Ed.), Emotion and social judgments (pp. 3-29). Oxford: Pergamon Press.

Forgas, J. P., \& Moylan, S. (1987). After the movies: The effects of mood on social judgments. Personality and Social Psychology Bulletin, 13, 467477.

Frijda, N. H. (1993). Mood, emotion episodes, and emotions. In M. Lewis \& J. M. Haviland (Ed.), Handbook of emotions (pp. 381-403). New York: Guilford Press.

Gaudino, J. L. (1988). The effect of vertical camera angle on perceptions of source credibility. Unpublished doctoral dissertation. Michigan State University.

Gerrard-Hesse, A., Spies, K., \& Hesse, F. W. (1994). Experimental inductions of emotional states and their effects: A review. British Journal of Psychology, 85, 55-78.

Grabe, M. E., Lang, A., \& Zhao, X. (2003). News content and form. Implications for memory and audience evaluations. Communication Research, 30, 387-413.

Grabe, M. E., Lombard, M., Reich, R. D., Bracken, C. C., \& Ditton, T. B. (1999). The role of screen size in viewer experiences of media content. Visual Communication Quarterly, 6(2), 4-9.

Grabe, M. E., Zhou, S., \& Barnett, B. (1998). Explicating sensationalism in television news: Content and the bells and whistles of form. Retrieved February 29, 2004, from http://list.msu.edu/cgibin/wa?A2=ind9812D\&L=aejmc\&P=1642. 
Grabe, M. E., Zhou, S., \& Barnett, B. (2001). Explicating sensationalism in television news: Content and the bells and whistles of form. Journal of Broadcasting \& Electronic Media, 45, 635-655.

Grabe, M. E., Zhou, S., Lang, A., \& Bolls, P. D. (2000). Packaging television news: The effects of tabloid on information processing and evaluative responses. Journal of Broadcasting \& Electronic Media, 44, 581-598.

Gross, J. J., \& Levenson, R. W. (1995). Emotion elicitation using films. Cognition and Emotion, 9, 87-108.

Hall, E. T. (1966): The hidden dimension: Man's use of space in public and private. London: Bodley Head.

Heijne, B. (2008, Februari 29). Geen leven zonder film [No life without movie], Retrieved April 17, 2008, from http://www.nrcboeken.nl/recensie/geenleven-zonder-film-0.

Hendriks Vettehen, P., Nuijten, C. M., \& Beentjes, J. W. J. (2005). News in an age of competition: Sensationalism in Dutch television news 1995-2001. Journal of Broadcasting \& Electronic Media, 49(3), 282-295.

Hirt, E. R., Melton, R. J., McDonald, H. E., \& Harackiewicz, J. M. (1996). Processing goals, task interest, and the mood performance relationship: A mediational analysis. Journal of Personality and Social Psychology, 71, 245-261.

Hoffman, B., \& Ritchie, D. (1997). Using multimedia to overcome the problems with problem based learning. Instructional Science, 25, 87-115.

Isen, A. M. (1984). Toward understanding the role of affect in cognition. In R. S. Wyer \& T. K. Srull (Eds.), Handbook of social cognition. Vol. 3 (pp. 179-236). Hillsdale, NJ: Lawrence Erlbaum Associates.

Isen, A. M. (2002). Missing in action in the AIM: Positive affect's facilitation of cognitive flexibility, innovation, and problem solving. Psychological Inquiry, 13(1), 57-65.

Isen, A. M., \& Daubman, K. A. (1984). The influence of affect on categorization. Journal of Personality and Social Psychology, 47, 1206-1217.

Isen, A. M., Johnson, M. M. S., Mertz, E., \& Robinson, G. F. (1985). The influence of positive affect on the unusualness of word associations. Journal of Personality and Social Psychology, 48, 1413-1426.

Isen, A. M., Daubman, K. A., \& Nowicki, G. P. (1987). Positive affect facilitates creative problem solving. Journal of Personality and Social Psychology, 52, 1122-1131.

Izard, C. E. (1977). Human emotions. New York: Plenum Press. 
Jacobson, R. L. (2008). How the rise of digital video is transforming education, eSchool News Special Report: Visual Learning, January 2, 2008.

Retrieved June 2, 2008, from http://www.eschoolnews.com/news/special-reports/.

Jonassen, D. H., Peck, K. L., \& Wilson, B. G. (1999). Learning with technology: a constructivist perspective. Upper Saddle River, NJ: Prentice Hall.

Kamins, M. A., Marks, L. J., \& Skinner, D. (1991). Television commercial evaluation in the context of program induced mood: Congruency versus consistency effects. Journal of Advertising, 20 (2), 1-14.

Kappas, A., Hess, U., Barr, C. L., \& Kleck, R. E. (1994). Angle of regard: The effect of the vertical viewing angle on the perception of facial expressions. Journal of Nonverbal Behavior, 18 (4), 263-280.

Kaufmann, G., \& Vosburg, S. K. (1997). "Paradoxical” mood effects on creative problem solving. Cognition and Emotion, 11, 151-170.

Kaufmann, G., \& Vosburg, S. K. (2002). The effects of mood on early and late idea production. Creativity Research Journal, 14, 317-330.

Kelley, M. (2007). The effect of screen size and audio delivery system on memory for television news. Visual Communication Quarterly, 14 (3), 176-188.

Keppell, M., Elliott, K., Kennedy, G., Elliott, S., \& Harris, P. (2003). Using authentic patients encounters in a medical curriculum. In S. Naido (Ed.), Learning \& teaching with technology: Principles and practices (pp. 85-96). London: Kogan Page.

Kim, J., \& Morris, J. D. (2007). The power of affective response and cognitive structure in product-trial attitude formation. Journal of Advertising, 36 (1), 95-106.

Klimesch, W. (1994). The structure of long-term memory: A connectivity model of semantic processing. Hillsdale, NJ: Erlbaum.

Koumi, J. (2006). Designing educational video and multimedia for open and distance learning. The Open and Flexible Learning Series. London: Taylor \& Francis.

Kozma, R. B. (1994). Will media influence learning? Reframing the debate. Educational Technology Research \& Development, 42 (2), 7-19.

Kraft, R. N. (1987). The influence of camera angle on comprehension and retention of pictorial events. Memory \& Cognition, 15 (4), 291-307.

Kress, G., \& van Leeuwen, T. (1996): Reading images: The grammar of visual design. London: Routledge.

Lang, A. (2000). The limited capacity model of mediated message processing. Journal of Communication, 50(1), 46-70. 
Lang, A., Bolls, P. D., Potter, R. F., \& Kawahara, K. (1999). The effects of production pacing and arousing content on the information processing of television messages. Journal of Broadcasting and Electronic Media, $43,451-475$.

Lang, A., Dhillon, P., \& Dong, Q. (1995). Arousal, emotion, and memory for television messages. Journal of Broadcasting and Electronic Media, 38, 1-15.

Lang, A., Shin, M., Bradley, S. D., Wang, Z., Lee, S., \& Potter, D. (2005). Wait! Don't turn that dial! More excitement to come! The effects of story length and production pacing in local television news on channel changing behavior and information processing in a free choice environment. Journal of Broadcasting \& Electronic Media, 49 (1), 3-22.

Liao, Y. C. \& Bright, G. W. (1991). Effects of computer programming on cognitive outcomes: A meta-analysis. Journal of Educational Computing Research, 7(3), 251-268.

Lipovetsky, G., \& Serroy, J. (2007). L'écran global: culture-médias et cinéma à l'âge hypermoderne. Paris: Le Seuil.

Lombard, M., Ditton, T. B., Grabe, M. E., \& Reich, R. D. (1997). The role of screen size in viewer responses to television fare. Communication Reports, 10(1), 95-106.

Lombard, M. (1995). Direct responses to people on the screen: Television and personal space. Communication Research, 22 (3), 288-324.

Lombard, M., Reich, R. D., Grabe, M. E., Bracken, C. C., \& Ditton, T. B. (1995, May). Big TVs, Little TVs: The Role of Screen Size in Viewer Responses to Point-of-view Movement. Paper presented to the Mass

Communication division at the annual conference of the International Communication Association, Albuquerque, NM.

Lombard, M., Reich, R. D., Grabe, M. E., Bracken, C. C., \& Ditton, T. B. (2000). Presence and television: The role of screen size. Human Communication Research, 26(1), 75-98.

MacDonald, C. J., Stodel, E. J., Farres, L. G., Breithaupt, K., \& Gabriel, M. A. (2001). A framework for web-based learning. Internet and Higher Education, 4, 9-30.

Maier, N. R. F. (1970). Problem solving and creativity in individuals and groups. Belmont, CA: Brooks/Cole.

Mandell, L. M., \& Shaw, D. L. (1973). Judging people in the news unconsciously: Effect of camera angle and bodily activity. Journal of Broadcasting, 17, 353-365. 
Martin, L. L., \& Clore, G. L. (Eds). (2001). Theories of mood and cognition. A user's guidebook. Mahwah, NJ: Erlbaum.

Martin, L. L., Ward, D. W., Achee, J. W., \& Wyer, R. S. (1993). Mood as input: People have to interpret the motivational implications of their moods. Journal of Personality and Social Psychology, 64, 317-326.

Martinsen, Ø. (1993). Insight problems revisited: The influence of cognitive styles and experience on creative problem solving. Creativity Research Journal, 6, 435-447.

Mayer, R. E. (2001). Multimedia learning. New York: Cambridge University Press.

Mayer, R. E., \& Moreno, R. (2003). Nine ways to reduce cognitive load in multimedia learning. Educational Psychologist, 38 (1), 43-52.

McCain, T. A., Chilberg, J., \& Wakshlag, J. (1977). The effect of camera angle on source credibility and attraction. Journal of Broadcasting, 21, 35-47.

McCain, T. A., \& Repensky, G. R. (1972). The Effect of Camera Shot on Interpersonal Attraction for Comedy Performers. Paper presented at 58th Annual Convention of the Speech Communication Association, Chicago, Illinois.

Mehrabian, A., \& Russell, J. A. (1974). An approach to environmental psychology. Cambridge, M.A.: MIT Press.

Messaris, P. (1997). Visual persuasion: The role of images in advertising. Thousand Oaks, CA: Sage.

Meyrowitz, J. (1986). Television and interpersonal behaviour: Codes of perception and response. In G. Gumpert \& R. Cathcart (Eds.), Inter/Media: Interpersonal communication in a media world (pp. 111132). New York/Oxford: Oxford University Press.

Mignault, A., \& Chaudhuri, A. (2003). The many faces of a neutral face: Head tilt and perception of dominance and emotion. Journal of Nonverbal Behavior, 27(2), 111-132.

Millerson, G. (1999). Television production (13 th ed). Oxford: Focal Press.

Moreno, R., \& Mayer, R. E. (1999). Cognitive principles of multimedia learning: The role of modality and contiguity. Journal of Educational Psychology, 91, 358-368.

Morrison, G. R. (1994). The media effects question: "Unresolvable" or asking the right question. Educational Technology Research and Development, $42(2), 41-44$.

Mraz, W., \& Runco, M. A. (1994). Suicide ideation and creative problem solving. Suicide and Life Threatening Behavior, 24, 38-47. 
Mundorf, N., Zillmann, D., \& Drew, D. (1991). Effects of disturbing televised events on the acquisition of information from subsequently presented commercials. Journal of Advertising, 20(1), 46-53.

Musch, J., \& Klauer, K. C. (2003). The psychology of evaluation: An introduction. In J. Musch \& K. C. Klauer (Eds.), The psychology of evaluation: Affective processes in cognition and emotion (pp. 1-5). Mahwah, NJ, Erlbaum.

Nielsen, J. (1997). TV meets the Web. Jakob Nielsen's Alertbox, February 15, 1997. Retrieved May 25, 2005, from http://www.useit.com/alertbox/9702b.html.

Nielsen, J. (2000). Designing web usability: The practice of simplicity. Indianapolis: New Riders Publishing.

Nielsen, J. (2005). Talking-head video is boring online. Jakob Nielsen's Alertbox, December 5, 2005. Retrieved April 16, 2006, from http://www.useit.com/alertbox/9702b.html.

Oblinger, D. G., \& Oblinger, J. L. (2005). Is it age or IT: First steps toward understanding the Net Generation. In D. G. Oblinger and J. L. Oblinger (Eds.), Educating the Net Generation (pp. 2.1-2.20). EDUCAUSE Ebook, retrieved November 2006, from http://www.educause.edu/content.asp?PAGE_ID=5989\&bhcp=1.

Park, J., Lennon, S. J., \& Stoel, L. (2005). On-line product presentation: effects on mood, perceived risk, and purchase intention. Psychology \& Marketing, 22(9), 695-719.

Paivio, A. (1986). Mental representations: A dual coding approach. Oxford: Oxford University Press.

Pekrun, R. (2005). Progress and open problems in educational emotion research. Learning and Instruction, 15, 497-506.

Persson, P. (1998). Towards a psychological theory of close-ups: Experiencing intimacy and threat. Retrieved September 22, 2004, from http:// www.arts.uwaterloo.ca/FINE/juhde/pers981.htm.

Persson, M., \& Calmhout, M. van, (2006, November 2). Iedereen wil plotseling ook een beetje Al Gore zijn. Retrieved April, 16, 2008, from http://www.volkskrant.nl/wetenschap/article364677.ece.

Philippot, P. (1993). Inducing and assessing differentiated emotion-feeling states in the laboratory. Cognition and Emotion, 7(2), 171-193.

Philips Research - Technologies. (2008). Ambilight - the new TV experience. Retrieved, July 12, 2008, from http://www.research.philips.com./technologies/syst_softw/ami/ ambilight.html. 
Piaget, J. (1952). The origins of intelligence in children. New York: International University Press.

Plutchik, R. (1980). Emotion: A psychoevoluntionary synthesis. New York: Harper and Row.

Rahim, A., \& Magner, N. R. (1995). Confirmatory factor analysis of the styles of handling interpersonal conflict: First-order factor model and its invariance across groups. Journal of Applied Psychology, 80, 122-132.

Ravaja, N., Kallinen, K., Saari, T., \& Keltikangas-Järvinen, L. (2004). Suboptimal exposure to facial expressions when viewing video messages from a small screen: Effects on emotion, attention, and memory. Journal of Experimental Psychology: Applied, 10, 120-131.

Ravaja, N. (2004). Effects of image motion on a small screen on emotion, attention, and memory: Moving-face versus static-face newscaster. Journal of Broadcasting \& Electronic Media, 48 (1), 708-733.

Ravaja, N., Saari, T., Kallinen, K., \& Laarni, J. (2006). The role of mood in the processing of media messages from a small screen: Effects on subjective and physiological responses. Mediapsychology, 8 (3), 239-265.

Reeves, B, \& Geiger, S. (1994). Designing experiments that assess psychological responses. In A. Lang (Ed.), Measuring psychological responses to media (pp. 165-180). Hillsdale, NJ: Lawrence Erlbaum Associates.

Reeves, B., \& Nass, C. I. (1996). The media equation: How people treat computers, television, and new media like real people and places. New York: Cambridge University Press.

Reeves, B., Detenber, B., \& Steuer, J. (1993). New Televisions: The Effects of Big Pictures and Big Sound on Viewer Responses to the Screen. Paper presented at the information Systems Division of the Conference of the International Communication Association, Washington, DC.

Reeves, B., Lang, A., Kim, E. Y., \& Tatar, D. (1999). The effects of screen size and message content on attention and arousal. Media Psychology, 1, 4967.

Reeves, B., Lombard, M., \& Melwani, G. (1992, May). Faces on the Screen: Pictures or Natural Experience? Paper presented to the Mass Communication division at the annual conference of the International Communication Association, Miami, FL.

Reiser, R. A. (1994). Clark's invitation to dance: An instructional designer's response. Educational Technology Research and Development, 42 (2), 45-48.

Reiser, R. A., \& Gagné, R. M. (1983). Selecting media for instruction. Englewood Cliffs, NJ: Educational Technology Publications. 
Reisslein, J., Seeling, P., \& Reisslein, M. (2005).Video in distance education. ITFS vs. web-streaming: Evaluation of student attitudes. Internet and Higher Education, 8(1), 25-44.

Roesch, S. C., (1999). Modelling the direct and indirect effects of positive emotional and cognitive traits and states on social judgements. Cognition and Emotion, 13(4), 387-418.

Romiszowski, A. J. (1988). The selection and use of instructional media. Second Edition. London: Kogan Page.

Ross, S. M. (1994). Delivery trucks or Groceries? More food for thought on whether media (will, may, can't) influence learning. Educational Technology Research and Development, 42 (2), 5-6.

Rottenberg, J., Ray, R. R., \& Gross, J. J. (2007). Emotion elicitation using films. In J. A. Coan \& J. J. B. Allen (Eds.), The handbook of emotion elicitation and assessment (pp. 9-28). New York: Oxford University Press.

Rubin, A. M. (1984). Ritualized and instrumental television viewing. Journal of Communication, 34 (4), 67-77.

Rushby, N. (1987). From trigger video to videodisc: a case study in interpersonal skills. In D. Laurillard (Ed.), Interactive media: Working methods and practical applications (pp. 116-131). Chichester: Ellis Horwood Publishers.

Russell, J. A. (1980). A circumplex model of affect. Journal of Personality and Social Psychology, 39(6), 1161-1178.

Salomon, G. (1979). Interaction of media cognition and learning. London: Jossey-Bass.

Salomon, G. (1984). Television is "easy" and print is "tough". The differential investment of mental effort in learning as a function of perceptions and attributions. Journal of Educational Psychology, 76 (4), 647-658.

Schwartz, N. (2001). Feelings as information: Implications for affective influences on information processing. In L. L. Martin \& G. L. Clore (Eds). Theories of mood and cognition. A user's guidebook (pp. 159176). Mahwah, NJ: Erlbaum.

Shapiro, R. Y. (1986). The effects of screen size and sensation seeking on physiological arousal and emotional state (doctoral dissertation). Dissertation Abstracts International, 47 (6-B), 2634.

Shephard, K. (2003). Questioning, promoting and evaluating the use of streaming video to support student learning. British Journal of Educational Technology, 34 (3), 295-308.

Simon, H. A. (1956). Rational choice and the structure of the environment. Psychological Review, 63, 129-138. 
Simons, R. F., Detenber, B. H., Reiss, J. E., \& Shults, C. W. (2000). Image motion and context: A between- and within-subjects comparison. Psychophysiology, 37, 706-710.

Simons, R. F., Detenber, B. H., Roedema, T. M., \& Reiss, J. E. (1999). Emotion processing in three systems: The medium and the message. Psychophysiology, 36, 619-627.

Smaldino, S. (1999). Instructional design for distance education. Techtrends, 43 (5), 9-13.

Stephens, M. (1998). The rise of the image; the fall of the word. Oxford: Oxford University Press.

Schiffenbauer, A., \& Schiavo, R. S. (1976). Physical distance and attraction: An intensification effect. Journal of Experimental Social Psychology, 12, 274-282.

Schwartz, B., Tesser, A., \& Powell, E. (1982). Dominance cues in nonverbal behavior. Social Psychology Quarterly, 45 (2), 114-120.

Tennyson, R. D. (1994). The big wrench vs. integrated approaches: The great media debate. Educational Technology, Research \& Development, 42 (3), 15-28.

Tiemens, R. (1970). Some relationships of camera angle to communicator credibility. Journal of Broadcasting, 14, 483-490.

Ullmer, E. J. (1994). Media and learning: Are there two kinds of truth? Educational Technology Research \& Development, 42 (1), 21-32.

Verhagen, P. W. (1996). Functions and design of video components in multimedia applications. In D. P. Ely \& B. B. Minor (Eds.), Educational Media and Technology Yearbook 1995/1996(pp. 105-118). Englewood (Co): Libraries Unlimited.

Verhagen, P. W., \& Verleur, R. (2007, Jan). Video: narratieve structuren en affectieve componenten. In P. W. Verhagen (Chair), Media nú: Nieuwe onderwijsmedia na 25 jaar. [Media now: New educational media after 25 years.] Media symposium conducted at the University of Twente, Enschede, The Netherlands.

Verleur, R., Verhagen, P. W., \& Heuvelman A. (2007). Can mood-inducing videos affect problem-solving activities in a web-based environment? British Journal of Educational Technology, 8 (6), 1010-1019.

Verleur, R., Verhagen, P. W., \& Heuvelman, A. (2008). Affective video on the web: Do medium and image size matter? Manuscript submitted for publication. 
Verleur, R., Verhagen, P. W., \& Heuvelman, A. (2008). Trigger videos on the web: Impact of audiovisual design. Manuscript submitted for publication.

Verwijs, C. A. (1998). A mix of core and complementary media: New perspectives in media-decision making. Doctoral dissertation, University of Twente, The Netherlands.

Vosburg, S. K., \& Kaufmann, G. (1999). Mood and creativity research: The view from a conceptual organizing perspective. In S. W. Russ (Ed.), Affect, creative experiences, and psychological adjustment (pp. 19-39). Philadelphia: Brunner/Mazel.

Vosburg, S. K. (1998). The effect of positive and negative mood on divergentthinking performance. Creativity Research Journal, 2, 165-172.

Weisglass, K. (2005). Image manipulation in political advertisements: How color and music influence viewer attitudes and emotions. Thesis, Haverford College, Haverford.

Wetzel, C. D., Radke, P. H., \& Stern, H.W. (1994). Instructional effectiveness of video media. New Jersey: Lawrence Erlbaum Associates, Inc.

Wurtzel, A. H., \& Dominick, J. R. (1971-72). An evaluation of television drama: Interaction of acting styles and shot selection. Journal of Broadcasting, $16(1), 103-110$.

Young, C, \& Asensio, M. (2002). Looking through three I's: The pedagogic use of streaming video. Proceedings of Networked Learning, Sheffield. 


\section{Appendices}

Appendix A Questionnaire Study 1

Appendix B Questionnaire Study 2

Appendix C Web Trigger Video "Library Problem"

Appendix D Questionnaire Study 3 


\section{Appendix A}

\section{Questionnaire Study 1}

The web page as presented on screen in the experimental web environment showing the questionnaire (in Dutch) that was presented after a video clip in the first study (Chapter 3).

\section{Videoclip 1}

\section{Vragenlijst affectieve en cognitieve responsen}

In de vragenlijst komen drie vragen aan de orde: Een vraag over je mening over de video, een vraag over de gevoelens die de video bij je oproept en een vraag over je huidige stemming.

$\mathrm{Om}$ het onderscheid tussen 'gevoel' en 'stemming' duidelijk te maken volgt hier een voorbeeld. Een directe reactie op de videoclip zou kunnen zijn dat deze bij jou positieve gevoelens oproept. Je stemming echter kan bijvoorbeeld 'neutraal' zijn (gebleven) of ook in meer- of mindere mate positief zijn (geworden).

Gebruik de schuifbalk (rechts van het scherm) om door de vragenlijst te lopen. Nadat je de vragenlijst hebt ingevuld, sluit je deze onderaan de pagina af.

Als je een toelichting wilt op het invullen van het antwoord: Klik dan op het help-icoon achter de betreffende vraag.

Wat is jouw MINING over de videoclip?
(geef dit voor elk onderdeel op de bijbehorende
\begin{tabular}{rllllllll|} 
zeer saai & 0 & 0 & 0 & 0 & 0 & 0 & 0 & zeer boeiend \\
zeer oppervlakkig & 0 & 0 & 0 & 0 & 0 & 0 & 0 & zeer diepgaand \\
zeer kort & 0 & 0 & 0 & 0 & 0 & 0 & 0 & zeer lang \\
zeer afwisselend & 0 & 0 & 0 & 0 & 0 & 0 & 0 & helemaal niet afwisselend \\
zeer zwaar & 0 & 0 & 0 & 0 & 0 & 0 & 0 & zeer licht \\
zeer goed & 0 & 0 & 0 & 0 & 0 & 0 & 0 & zeer slecht
\end{tabular}

Welke GEVOELENS roept de videoclip bij je op?
$\begin{array}{rllllllll}\text { (geef dit voor elk onderdeel op de bijbehorende } & 7-\text { puntsschaal aan s.v.p.) ? } \\ \text { zeer somber } & 0 & 0 & 0 & 0 & 0 & 0 & 0 & \text { zeer vrolijk } \\ \text { zeer blij } & 0 & 0 & 0 & 0 & 0 & 0 & 0 & \text { helemaal niet blij } \\ \text { zeer droevig } & 0 & 0 & 0 & 0 & 0 & 0 & 0 & \text { helemaal niet droevig } \\ \text { zeer aangenaam } & 0 & 0 & 0 & 0 & 0 & 0 & 0 & \text { zeer onaangenaam } \\ \text { zeer positief } & 0 & 0 & 0 & 0 & 0 & 0 & 0 & \text { zeer negatief } \\ \text { zeer betrokken } & 0 & 0 & 0 & 0 & 0 & 0 & 0 & \text { helemaal niet betrokken }\end{array}$


Appendices

\section{(web page continued)}

\begin{tabular}{|c|c|c|c|c|c|c|c|c|}
\hline $\begin{array}{l}\text { Geef aan in welke STEM } \\
\text { (geef dit voor elk onderde }\end{array}$ & MII & & & & & & & s.v.p.) ? \\
\hline zeer opgewonden & 0 & 0 & 0 & 0 & 0 & 0 & 0 & zeer kalm \\
\hline zeer uitgelaten & 0 & 0 & 0 & 0 & 0 & 0 & 0 & helemaal niet uitgelaten \\
\hline zeer rustig & 0 & 0 & 0 & 0 & 0 & 0 & 0 & zeer onrustig \\
\hline zeer negatief & 0 & 0 & 0 & 0 & 0 & 0 & 0 & zeer positief \\
\hline zeer aangenaam & 0 & 0 & 0 & 0 & 0 & 0 & 0 & zeer onaangenaam \\
\hline zeer vrolijk & 0 & 0 & 0 & 0 & 0 & 0 & 0 & zeer somber \\
\hline
\end{tabular}

In het veld hieronder kun je eventuele opmerkingen noteren en/of toelichtingen geven op de bovenstaande vragen. [?]

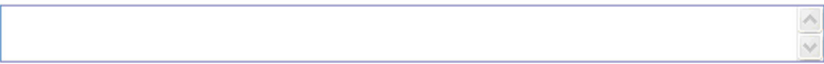

\section{Afsluiten Videoclip 1}

Wanneer je alle vragen hebt ingevuld, klik dan op "Doorgaan" om de gegevens op te slaan en naar het volgende onderdeel van het onderzoek te gaan.

Doorgaan 


\section{Appendix B}

\section{Questionnaire Study 2}

The web page as presented on screen in the experimental web environment showing the questionnaire (in Dutch) that was presented after a video clip in the second study (Chapter 4).

\section{Experiment}

Vragen over de videoclip

Deze vragenlijst bestaat uit drie vraagblokjes waarin naar jouw reactie op de videoclip wordt gevraagd. Als je de vragen hebt ingevuld, klik dan op "Doorgaan" en de opdracht met de probleembeschrijving verschijnt.

1) Geef voor elk van de drie onderstaande visuele schalen aan welke figuur het meest overeenkomt met de wijze waarop jij je nu voelt. ??

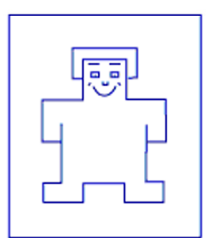

0

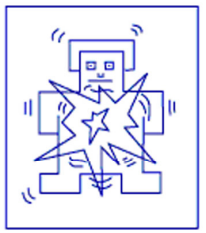

0

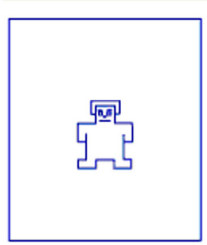

o

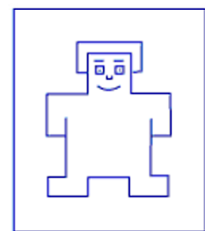

O

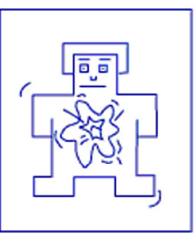

O

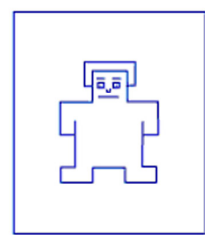

O

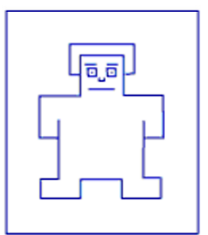

0

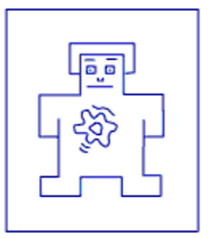

O

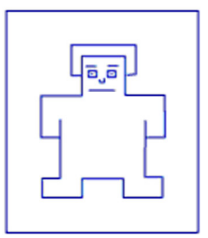

O

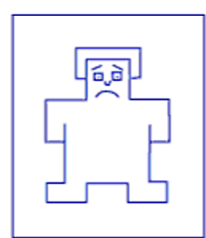

○

O

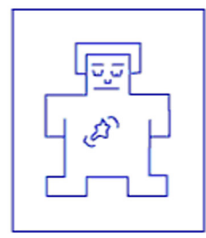

O

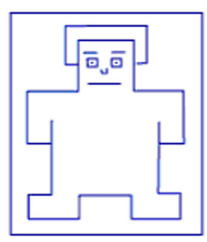

0

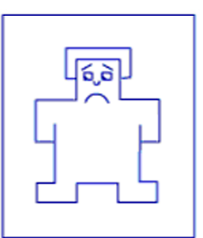

0

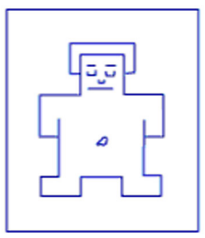

0

0

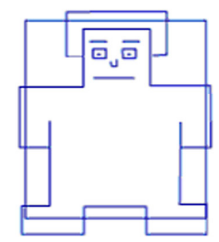

O

○ 
Appendices

\section{(web page continued)}

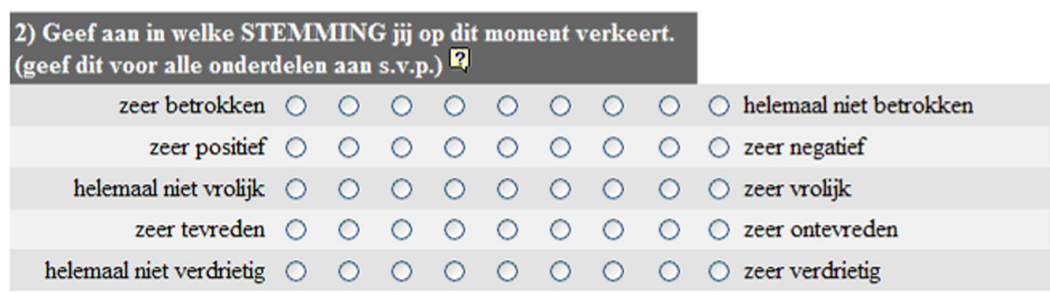

\section{3) Wat is jouw MENING over de videoclip?}

(geef dit voor alle onderdelen aan s.v.p.) ?

$\begin{array}{rllllllllll}\text { zeer slecht } & 0 & 0 & 0 & 0 & 0 & 0 & 0 & 0 & 0 & \text { zeer goed } \\ \text { zeer boeiend } & 0 & 0 & 0 & 0 & 0 & 0 & 0 & 0 & 0 & \text { zeer saai } \\ \text { zeer vervelend } & 0 & 0 & 0 & 0 & 0 & 0 & 0 & 0 & 0 & \text { helemaal niet vervelend } \\ \text { zeer onaangenaam } & 0 & 0 & 0 & 0 & 0 & 0 & 0 & 0 & 0 & \text { zeer aangenaam }\end{array}$

Wanneer je alle vragen hebt ingevuld, klik dan op "Doorgaan" om de gegevens op te slaan en naar het volgende onderdeel van het onderzoek te gaan.

Doorgaan 


\section{Appendix C}

\section{Web Trigger Video "Library Problem"}

Synopsis, script and shot lists for the web trigger video "Library problem" as applied in the third study (Chapter 5).

\section{Synopsis for the web trigger video "Library Problem"}

Title: Library problem

Program length: 1 minute

Distribution: Web (QuickTime, Playback at computer facilities of TO, LAN)

Purpose:

The purpose of the video is to show the library problem and to evoke a strong emotional response in the viewer with regard to the problem ('trigger video').

Context: A web page introduces briefly the "Library problem". After that, the trigger video is presented in a video window. The video clip ends at the climax of the scene when the main character is about to act. At this point the participant is asked to select from a list of options the most likely action for the main character.

Video content:

The video clip consists of one scene that shows a realistic problem situated in a library. Ralph is sitting in the library. He concentrates on his study book in preparation for tomorrow's exam. He sits in the library because it is a zone of silence where he can work undisturbed. His fellow student, Ron, enters. Ron sees Ralph, comes to his table and sits adjacent to him. Ron is making noise and wants to talk to Ralph. He is obviously disturbing Ralph with his study. Ralph feels increasingly uncomfortable with the situation up to the moment that it is time to act.

\section{Script for the web trigger video}

Ralph is sitting in a library. He concentrates on his study book in preparation for tomorrow's exam. He sits in the library because it is a zone of silence where he can work undisturbed. 
His fellow student, Ron, enters. Ron sees Ralph, comes to his table and says too loudly:

\section{"Hi, Ralph, how are things going?"}

Meanwhile he noisily pulls out a chair and smashes his bag into it.

Ralph answers: "I am fine. But can you please be quiet?"

Ralph focuses on his book again while Ron sits down on the chair adjacent to the chair with his bag. He takes a few books and other paperwork from his bag, which he drops with a crash on the table.

Ralph: "Ron, please, I need this time to prepare for the exam."

And again Ralph concentrates on his book.

Ron: $\quad$ "OK, OK..."

Ron opens one of his books and a note pad. He bends to his bag to look for a pen. He not only takes a pen from his bag but also an envelope with photos inside.

Ron to Ralph: "Hey, Ralph. I have the photos here from our last field trip."

Ralph lifts his head and looks annoyed.

Ron: "Sorry."

Ralph resumes his study. While he is reading and taking notes, Ron looks at the photos which make him laugh loudly now and then and say things like:

\section{"Look at that. Wow!"}

Ralph looks more and more unhappy.

All of a sudden Ron holds out his hand to show Ralph a certain photo, saying:

\section{"This is terrific. Ralph you really should see this one!"}

Ralph puts down his pen and faces Ron. It is clear that he is now ready to act. 
Representative shots for the three versions of the web trigger video

\begin{tabular}{|c|c|c|c|c|}
\hline Shot & Scene description & $\begin{array}{l}\text { Neutral } \\
\text { version }\end{array}$ & $\begin{array}{l}\text { Close-up } \\
\text { Version }\end{array}$ & $\begin{array}{l}\text { Dominance } \\
\text { version }\end{array}$ \\
\hline 1 & $\begin{array}{l}\text { [2-shot] } \\
\text { Ralph is sitting in a library. } \\
\text { He concentrates on his study } \\
\text { book in preparation for } \\
\text { tomorrow's exam. He sits in } \\
\text { the library because it is a } \\
\text { zone of silence where he can } \\
\text { work undisturbed. } \\
\text { His fellow student, Ron, } \\
\text { enters. Ron sees Ralph, } \\
\text { comes to his table, and says } \\
\text { too loudly: } \\
\text { "Hi, Ralph, how are things } \\
\text { going?" } \\
\text { Meanwhile he noisily pulls } \\
\text { out a chair and smashes ... }\end{array}$ & $\begin{array}{l}\text { TOTAL } \\
\text { की }\end{array}$ & 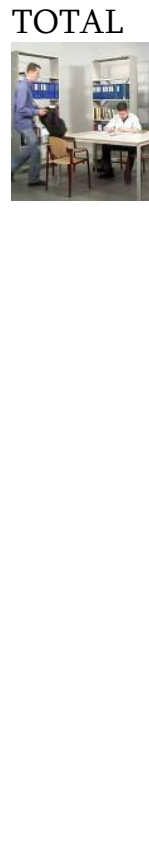 & 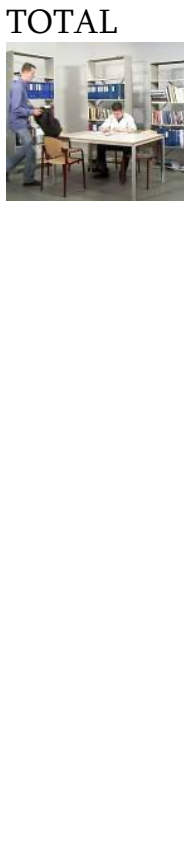 \\
\hline 2 & $\begin{array}{l}\text { [.. his bag into it. } \\
\text { Ralph answers: "I am fine. } \\
\text { But can you please be } \\
\text { quiet?" } \\
\text { Ralph focuses on his book } \\
\text { again while Ron sits down } \\
\text { on the chair adjacent to the } \\
\text { chair with his bag. He takes } \\
\text { a few books and other } \\
\text { paperwork from his bag, } \\
\text { which he drops with a } \\
\text { crash... }\end{array}$ & TS & TS & TS \\
\hline
\end{tabular}




\begin{tabular}{|c|c|c|c|c|}
\hline 3 & $\begin{array}{l}\text {... on the table } \\
\text { Ralph: "Ron, please, I need } \\
\text { this time to prepare for the } \\
\text { exam." } \\
\text { And again Ralph } \\
\text { concentrates on his book. }\end{array}$ & MS & $\mathrm{CU}$ & H-MS \\
\hline 4 & $\begin{array}{l}\text { Ron: “OK, OK..." } \\
\text { Ron opens one of his books } \\
\text { and a note pad. He bends to } \\
\text { his bag to look for a pen. He } \\
\text { not only takes a pen from } \\
\text { his bag but also an envelope } \\
\text { with photos inside. } \\
\text { (Ralph looks disturbed by } \\
\text { the noise while studying) } \\
\text { Ron to Ralph: } \\
\text { "Hey, Ralph. I have the } \\
\text { photos... }\end{array}$ & MS & MS & $\mathrm{L}-\mathrm{CU}$ \\
\hline 5 & $\begin{array}{l}\text { [Ralph] } \\
\text {...here from our last field } \\
\text { trip." } \\
\text { Ralph lifts his head and } \\
\text { looks annoyed. } \\
\text { Ron: "Sorry." } \\
\text { Ralph resumes his study. } \\
\text { While he is reading and } \\
\text { taking notes, Ron looks at } \\
\text { the photos which make him } \\
\text { laugh loudly now and ... }\end{array}$ & ITK & $\mathrm{CU}$ & $\mathrm{H}-\mathrm{MS}$ \\
\hline
\end{tabular}




\begin{tabular}{|c|c|c|c|c|}
\hline 6 & $\begin{array}{l}\text { [Ron] } \\
\text {.... then and say things like: } \\
\text { "Look at that. Wow!" } \\
\text { Ralph looks more and more } \\
\text { unhappy. } \\
\text { All of a sudden Ron holds } \\
\text { out his hand to show Ralph } \\
\text { a certain photo, saying: } \\
\text { "This is terrific. Ralph you } \\
\text { really should see... }\end{array}$ & MS & MS & $\frac{\mathrm{L}-\mathrm{CU}}{\mathrm{D}}$ \\
\hline 7 & $\begin{array}{l}\text {...this one!" } \\
\text { Ralph puts down his pen } \\
\text { and faces Ron. It is clear that } \\
\text { he is now ready to act. }\end{array}$ & MS & $\mathrm{X}-\mathrm{CU}$ & H-MS \\
\hline
\end{tabular}

Note:

TOTAL: Total shot: Establishing shot of the scene

TS: $\quad$ Two shot: Introducing the two characters

MS: $\quad$ Medium shot

H-MS: $\quad$ Medium shot from high camera position

L-CU: $\quad$ Close-up shot from low camera position

CU: $\quad$ Close-up shot

X-CU: $\quad$ Extreme close-up shot 


\section{Appendix D}

\section{Questionnaire Study 3}

The web page as presented on screen in the experimental web environment showing the questionnaire (in Dutch) that was presented after a video clip in the third study (Chapter 5).

\section{Vragenlijst 1}

De eerste vragen gaan over RALF (degene die zit te studeren).

1) Geef voor elk van de drie onderstaande visuele schalen aan welke figuur het meest overeenkomt met hoe RALF zich nu voelt. ?

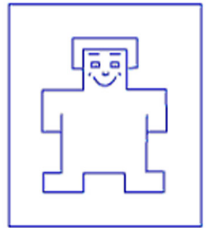

○

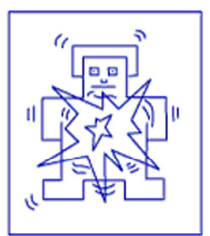

O

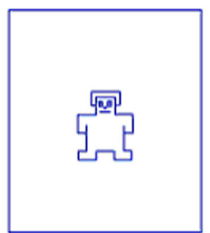

○

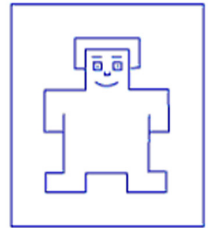

○

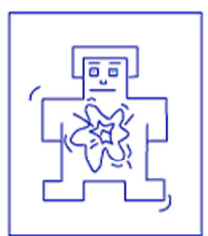

O

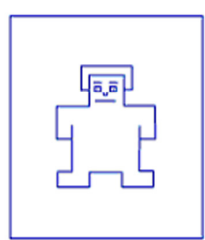

O

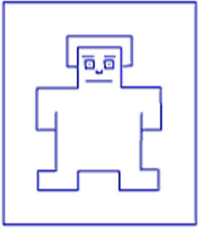

O

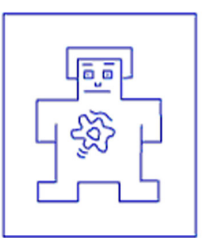

○

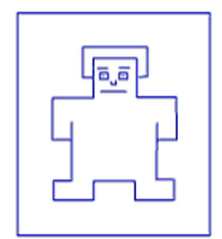

O
○

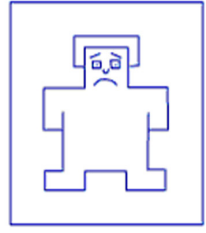

○

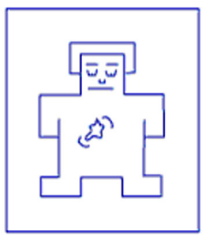

O

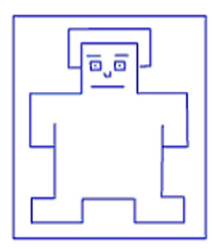

O

o
O
O

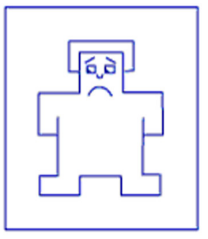

o

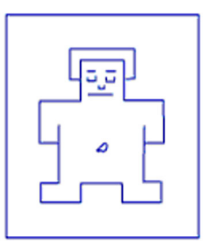

o 0

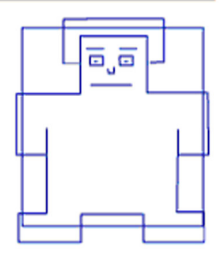

○

O 


\section{(web page continued)}

2) Hoe denk je dat RALF nu gaat reageren (klik één van de alternatieven aan)?

Hij negeert Ron verder in de hoop dat hij de hint oppakt

OHij negeert Ron om ruzie te voorkomen

Hij gebruikt een excuus om weg te gaan om ergens anders te gaan studeren

Hij loopt boos weg

OHij laat Ron z'n verhaal doen, in de hoop dat hij daarna kan studeren

O Hij zegt wat hij vindt/voelt en stelt Ron voor een andere keer de foto's te bekijken

O Hij stelt voor dat Ron kort een paar foto's laat zien, zodat hij snel verder kan met studeren

O Hij negeert Ron nadrukkelijk, zodat Ron uiteindelijk ophoudt of weggaat

Hij blijft vriendelijk, maar eist dat Ron ophoudt of weggaat

O Hij wordt boos en eist dat Ron ophoudt of weggaat

OHij wordt agressief en eist dat Ron ophoudt of weggaat

Anders, namelijk (vul in):

3) Gebruik de onderstaande schalen om aan te geven wat jij van RALF vindt (OF welke indruk je van RALF hebt). (geef dit voor alle onderdelen aan s.v.p.) ??

\begin{tabular}{|rllllllllll}
\hline zeer vriendelijk & 0 & 0 & 0 & 0 & 0 & 0 & 0 & 0 & 0 & zeer onvriendelijk \\
zeer onsympathiek & 0 & 0 & 0 & 0 & 0 & 0 & 0 & 0 & 0 & zeer sympathiek \\
zeer onderdanig & 0 & 0 & 0 & 0 & 0 & 0 & 0 & 0 & 0 & zeer dominant \\
zeer eigenzinnig & 0 & 0 & 0 & 0 & 0 & 0 & 0 & 0 & 0 & zeer meegaand \\
zeer emotioneel & 0 & 0 & 0 & 0 & 0 & 0 & 0 & 0 & 0 & zeer (emotioneel) stabiel \\
zeer koel & 0 & 0 & 0 & 0 & 0 & 0 & 0 & 0 & 0 & zeer warm \\
zeer ongevoelig & 0 & 0 & 0 & 0 & 0 & 0 & 0 & 0 & 0 & zeer gevoelig \\
zeer berekenend & 0 & 0 & 0 & 0 & 0 & 0 & 0 & 0 & 0 & zeer open \\
zeer sociaal gericht & 0 & 0 & 0 & 0 & 0 & 0 & 0 & 0 & 0 & zeer op zichzelf gericht \\
zeer ontspannen & 0 & 0 & 0 & 0 & 0 & 0 & 0 & 0 & 0 & zeer gespannen \\
zeer zelfverzekerd & 0 & 0 & 0 & 0 & 0 & 0 & 0 & 0 & 0 & zeer onzeker \\
zeer opportunistisch & 0 & 0 & 0 & 0 & 0 & 0 & 0 & 0 & 0 & zeer conformistisch \\
zeer impulsief & 0 & 0 & 0 & 0 & 0 & 0 & 0 & 0 & 0 & zeer beheerst \\
zeer serieus & 0 & 0 & 0 & 0 & 0 & 0 & 0 & 0 & 0 & zeer enthousiast \\
zeer voorzichtig & 0 & 0 & 0 & 0 & 0 & 0 & 0 & 0 & 0 & zeer onbevreesd \\
zeer traditioneel & 0 & 0 & 0 & 0 & 0 & 0 & 0 & 0 & 0 & zeer innovatief \\
zeer goed van & 0 & 0 & 0 & 0 & 0 & 0 & 0 & 0 & 0 & zeer wantrouwend \\
vertrouwen & 0 & & 0 & 0 & 0 & 0 & 0 & 0 & 0 & zeer fantasierijk \\
zeer nuchter & 0 & 0 & 0 & 0 & 0 & 0 & 0 & 0 & 0 & zeer abstract denkend \\
zeer concreet denkend & 0 & 0 & 0 & 0 & 0 & 0 & 0 &
\end{tabular}


Appendices

(web page continued)

De volgende vragen gaan over RON (degene die langs komt)

4) Geef voor elk van de drie onderstaande visuele schalen aan welke figuur het meest overeenkomt met hoe RON zich voelde. ?

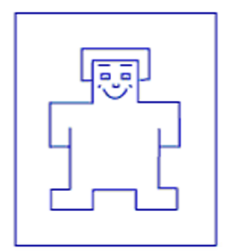

O

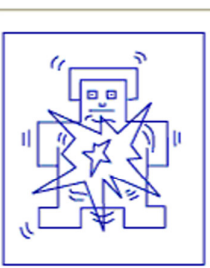

O

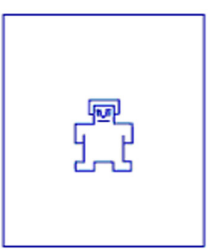

O

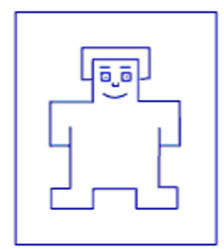

O

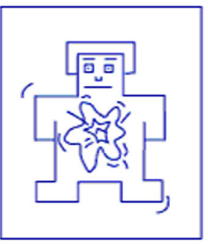

O

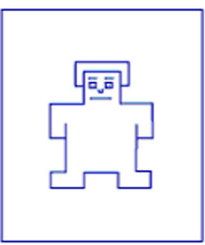

O

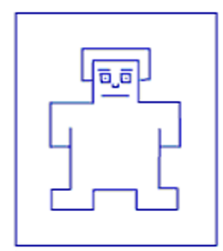

O

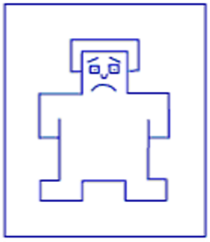

O

0

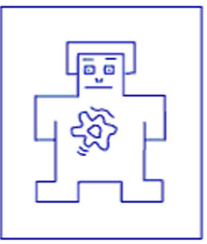

O

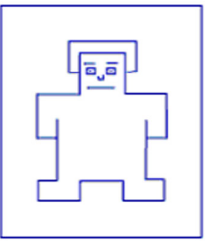

0

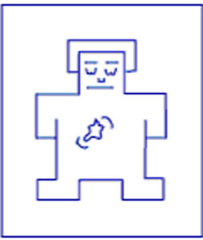

0

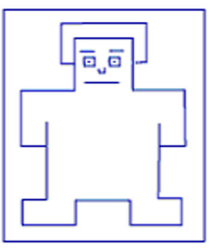

0

O

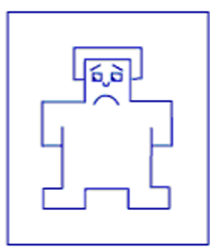

$0 \quad 0$

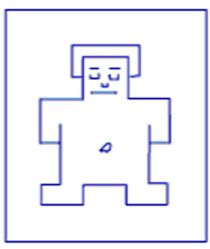

$0 \quad 0$

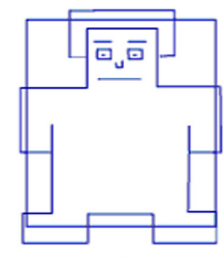

$0 \quad 0$ 


\section{(web page continued)}

5) Gebruik de onderstaande schalen om aan te geven wat jij van RON vindt (OF welke indruk je van RON hebt). (geef dit voor alle onderdelen aan s.v.p.) ?

\begin{tabular}{|rllllllllll}
\hline zeer vriendelijk & 0 & 0 & 0 & 0 & 0 & 0 & 0 & 0 & 0 & zeer onvriendelijk \\
zeer onsympathiek & 0 & 0 & 0 & 0 & 0 & 0 & 0 & 0 & 0 & zeer sympathiek \\
zeer onderdanig & 0 & 0 & 0 & 0 & 0 & 0 & 0 & 0 & 0 & zeer dominant \\
zeer eigenzinnig & 0 & 0 & 0 & 0 & 0 & 0 & 0 & 0 & 0 & zeer meegaand \\
zeer emotioneel & 0 & 0 & 0 & 0 & 0 & 0 & 0 & 0 & 0 & zeer (emotioneel) stabiel \\
zeer koel & 0 & 0 & 0 & 0 & 0 & 0 & 0 & 0 & 0 & zeer warm \\
zeer ongevoelig & 0 & 0 & 0 & 0 & 0 & 0 & 0 & 0 & 0 & zeer gevoelig \\
zeer berekenend & 0 & 0 & 0 & 0 & 0 & 0 & 0 & 0 & 0 & zeer open \\
zeer sociaal gericht & 0 & 0 & 0 & 0 & 0 & 0 & 0 & 0 & 0 & zeer op zichzelf gericht \\
zeer ontspannen & 0 & 0 & 0 & 0 & 0 & 0 & 0 & 0 & 0 & zeer gespannen \\
zeer zelfverzekerd & 0 & 0 & 0 & 0 & 0 & 0 & 0 & 0 & 0 & zeer onzeker \\
zeer opportunistisch & 0 & 0 & 0 & 0 & 0 & 0 & 0 & 0 & 0 & zeer conformistisch \\
zeer impulsief & 0 & 0 & 0 & 0 & 0 & 0 & 0 & 0 & 0 & zeer beheerst \\
zeer serieus & 0 & 0 & 0 & 0 & 0 & 0 & 0 & 0 & 0 & zeer enthousiast \\
zeer voorzichtig & 0 & 0 & 0 & 0 & 0 & 0 & 0 & 0 & 0 & zeer onbevreesd \\
zeer traditioneel & 0 & 0 & 0 & 0 & 0 & 0 & 0 & 0 & 0 & zeer innovatief \\
zeer goed van & 0 & 0 & 0 & 0 & 0 & 0 & 0 & 0 & 0 & zeer wantrouwend \\
vertrouwen & 0 & & 0 & 0 & 0 & 0 & 0 & 0 & 0 & zeer fantasierijk \\
zeer nuchter & 0 & 0 & 0 & 0 & 0 & 0 & 0 & 0 & 0 & zeer abstract denkend \\
zeer concreet denkend & 0 & 0 & 0 & 0 & 0 & 0 & 0 &
\end{tabular}

\section{Vraag over de videoclip}

6) Wat is jouw MENING over de VIDEOCLIP? (geef dit voor alle onderdelen aan s.v.p.) ?

$\begin{array}{rllllllllll}\text { zeer slecht } & 0 & 0 & 0 & 0 & 0 & 0 & 0 & 0 & 0 & \text { zeer goed } \\ \text { zeer boeiend } & 0 & 0 & 0 & 0 & 0 & 0 & 0 & 0 & 0 & \text { zeer saai } \\ \text { zeer vervelend } & 0 & 0 & 0 & 0 & 0 & 0 & 0 & 0 & 0 & \text { helemal niet vervelend } \\ \text { heel leuk } & 0 & 0 & 0 & 0 & 0 & 0 & 0 & 0 & 0 & \text { helemal niet leuk }\end{array}$

Wanneer je alle vragen hebt ingevuld, klik dan op "Doorgaan" om naar het volgende onderdeel van het onderzoek te gaan. 


\section{Samenvatting}

Ontwikkelingen in computer- en internettechnologie en de groeiende aandacht voor emoties leiden tot een toename in en een verscheidenheid aan toepassingen van affectieve video in webgebaseerde leeromgevingen. Dit proefschrift onderzoekt of video in een webomgeving effectief kan zijn in het oproepen van affectieve responsen en hoe deze responsen de takuitvoering kunnen beïnvloeden. Affectieve respons is daarbij gedefineerd als een emotionele respons of stemming, gemeten met subjectieve schalen op een valentie (positief-negatief) dimensie. Vier onderzoeksvragen zijn in drie experimentele studies onderzocht. In deze experimenten is de rol van drie presentatievariabelen van video op affectieve responsen en taakuitvoering onderzocht: videoweergave (medium, beeldgrootte), video-inhoud (positief, negatief) en videovormgeving (audiovisuele ontwerpvariabelen).

\section{Achtergronden}

Vroeger speelde in onze cultuur het gedrukte woord nog de grootste rol. Tegenwoordig leven we in een wereld die gedomineerd wordt door bewegende beelden. Video komt ons leven binnen door televisie, bioscoop, mobiele telefoons, iPods, game consoles en computer applicaties, met een prominente plaats voor het internet. YouTube, pas sinds 2005 actief, is een voorbeeld van een populaire video site. Tegelijkertijd zijn de inhoud en de vorm van boodschappen veranderd. Beelden zijn affectiever geworden. Zij bevatten meer emotionele inhoud en worden dramatischer gepresenteerd dan enkele jaren geleden. Ook is de weergave van videobeelden zowel veel groter (IMAX) als veel kleiner (mobiele telefoons) geworden.

De vraag is welke effecten deze veranderingen hebben op videogebruikers in een onderwijssetting. Uit klassiek experimenteel onderzoek op het gebied van stemming en cognitie blijkt dat door video opgeroepen affect de takkuitvoering kan beïnvloeden. In dat onderzoek speelt de positieve/negatieve aard ("valentie") van de video een bepalende rol, terwijl de video-inhoud niet aan de taak gerelateerd is: video wordt puur ingezet om affectieve reacties op te 
roepen. In de onderwijskundige (onderzoek)literatuur is de laatste jaren een groeiende belangstelling waar te nemen voor de rol van affect op het leerproces. Echter, bij onderwijskundig gebruik zijn de video-inhoud en de taak doorgaans wel gerelateerd aan elkaar. Deze technologische en onderwijskundige vernieuwingen vormen het kader voor dit proefschrift. Het onderzoek bevindt zich op het kruispunt van onderwijskundige technologie, mediapsychologie, video ontwerp en experimenteel onderzoek naar affect en cognitie.

De centrale vraagstelling van het proefschrift is: "Hoe beïnvloeden affectieve responsen op video de takuitvoering in een webomgeving?"Deze vraagstelling is in het theoretisch deel van dit proefschrift uitgewerkt tot de volgende vier onderzoeksvragen:

1) Produceren (positieve en negatieve) affectieve videomaterialen vergelijkbare affectieve responsen wanneer zij via een webgebaseerde omgeving worden aangeboden in vergelijking met een televisiesetting?

2) Heeft beeldgrootte invloed op affectieve responsen?

3) Beïnvloeden stemmingsinducerende videoclips daaropvolgende ongerelateerde probleemoplostaken wanneer beide in een webgebaseerde omgeving worden aangeboden?

4) Beinvloedt audiovisueel ontwerp kijkerresponsen op (trigger) videoinhoud en gerelateerde taakactiviteiten wanneer beide gepresenteerd worden in een webgebaseerde omgeving?

\section{Overzicht van de Studies}

In de eerste studie, die beschreven wordt in Hoofdstuk 3, wordt de effectiviteit van videoweergave in een webomgeving onderzocht: De eerste onderzoeksvraag exploreert of positieve en negatieve videomaterialen in een webgebaseerde omgeving vergelijkbare affectieve responsen produceren als in een televisiesetting. Deze laatste setting fungeert als referentiestandaard, omdat ouder video-onderzoek meestal daarop is gebaseerd. Beide mediasettings waren even effectief in het oproepen van emotionele valentie en in het induceren van stemming in deelnemers. De tweede onderzoeksvraag onderzoekt het effect van beeldgrootte op affectieve responsen op positieve en negatieve video-inhoud. Grotere beelden produceerden significant meer intense emotionele valentie en stemmingsveranderingen voor affectieve video-inhouden in gecombineerde 
mediasettings. Ook binnen de websetting is dit effect duidelijk aangetoond voor de positieve videoclip.

In de tweede studie, die beschreven wordt in Hoofdstuk 4, wordt eerst de video-inhoud gemanipuleerd om een (positieve of negatieve) stemming in deelnemers te induceren, waarna zij probleemoplostaken moeten uitvoeren. Deze taken zijn niet gerelateerd aan de video-inhoud. Het effect op de taakuitvoering wordt hierbij gemeten. Op basis van eerdere studies was de verwachting dat probleemoplossingsactiviteiten in een geoptimaliseerde (optimising) taakconditie (dit wil zeggen: het probleem is opgelost wanneer de oplossing voldoet aan de objectieve oplossingscriteria) door een negatieve stemming zouden worden bevorderd en dat probleemoplossingsactiviteiten in een tevreden (satisficing) taakconditie (dit wil zeggen: het probleem is opgelost als men tevreden is met de gevonden oplossing[en]) bevorderd zouden worden door een positieve stemming. Het experiment ondersteunt de verwachtingen voor de optimising conditie: het zien van een negatieve clip verbetert, en het zien van de positieve clip verslechtert de uitvoering op de inzichttaak. De verwachting voor de satisficing conditie wordt niet ondersteund: de positieve video clip heeft geen invloed op de uitkomsten van de divergente-denktaak. Samenvattend bewijst deze studie wel dat stemmingsinducerende videoclips daaropvolgende ongerelateerde probleemoplostaken kunnen beïnvloeden, wanneer beide worden weergegeven in een webomgeving.

De derde studie heeft betrekking op onderzoeksvraag 4. Deze studie wordt beschreven in Hoofdstuk 5. In het experiment wordt onderzocht of audiovisuele vormgeving kijkerreacties op video-inhoud en gerelateerde taakactiviteiten beïnvloedt, wanneer beide in een webomgeving worden weergegeven. Voor dit doel wordt een trigger video gebruikt, die een conflictsituatie toont tussen twee personages. Een trigger video eindigt op een emotionele climax. Het open einde roept affectieve reacties en gedachten op over mogelijke oplossingen voor de getoonde probleemsituatie. In de gebruikte audiovisuele vormgeving is horizontale afstand (nabijheid) en verticale afstand (ongelijkheid) in de relatie tussen de personages gemanipuleerd. Drie versies zijn ontwikkeld en vergeleken: een neutrale versie en twee emotionele versies (een close-up versie en een dominantie versie). De audiovisuele vormgeving blijkt waargenomen emotionele valentie en persoonlijkheidsevaluatie van de personages te beïnvloeden. 


\section{Conclusies}

In deze studie staat affectieve video op het web centraal. Video blijkt in een webomgeving net zo effectief te zijn in het oproepen van affectieve responsen als in een televisiesetting. De onderzoeksresultaten geven verder aan dat: (a) deze door video opgeroepen affectieve responsen een verschillende impact hebben op de taakuitvoering, en (b) de webomgeving zelf geen impact heeft op de affectieve responsen op video, maar presentatievariabelen, zoals beeldgrootte en videovormgeving het affectieve potentieel van video en dus de relatie tussen door video opgeroepen affectieve responsen en taakuitvoering beïnvloeden.

Het affectieve potentieel van video lijkt voornamelijk bepaald te worden door de audiovisuele kenmerken van video of symbool systemen en niet door het medium. Het medium is slechts een vervoermiddel. Dit impliceert dat webapplicaties een goed podium vormen om het affectieve potentieel van video te exploiteren. Het onderwijs volgt doorgaans de mediatrends: net zoals in het dagelijks leven zullen videoschermen zowel veel groter als veel kleiner worden. De bevindingen van deze studie suggereren dat video ook een affectief potentieel heeft wanneer kleinere schermen worden gebruikt. Dit is veelbelovend voor onderwijstoepassingen die typisch kleinere schermen gebruiken, zoals iPod-achtige toepassingen, mobiele telefoons of mini tv's. Onze studie suggereert echter wel dat als het doel is affectieve responsen op te roepen, grotere schermen effectiever zijn dan kleinere schermen. Uit het onderzoek blijkt namelijk dat presentatievariabelen van video die fysiek of psychologisch de video-inhoud vergroten, zoals beeldgrootte, en camerashotlengte (kadrering) en camerahoogte, een accentuerings- of intensiferingseffect hebben op affectieve responsen en evaluaties van deze inhoud.

\section{Discussie}

In dit onderzoeksproject stond de taakuitvoering centraal. Uit alle drie de studies komt naar voren dat de door video opgeroepen affectieve responsen doorwerkten op de taakuitvoering. De manier waarop de affectieve video doorwerkte op de taakuitvoering verschilt echter per studie. De eerste studie geeft inzicht in een contrasteffect. een eerder getoonde affectieve video beïnvloedt de affectieve responsen op een daaropvolgende video in de 
webomgeving. Na een negatieve video zijn de responsen op een neutrale video veel positiever dan na een positieve video (en vice versa). De tweede studie, waarin de probleemoplostaken centraal staan, geeft inzicht in een taakafhankelijk effect. bepaalde probleemoplostaken worden beter uitgevoerd in bepaalde stemmingen. In onze studie wordt de inzichttaak beter uitgevoerd na het zien van een negatieve video dan na het zien van een positieve video. De derde studie geeft inzicht in de impact van een (emotionele) audiovisuele vormgeving op taken gerelateerd aan de inhoud van de video. Bij de persoonsbeoordelingen, inclusief de waargenomen emotionele valentie van de videopersonages, is een accentuatie-effect gevonden. De interpretatie van de scene lijkt op een affectcongruente manier beïnvloed te worden: een meer negatief vormgegeven video resulteert in een negatievere interpretatie van de persoon en de gepresenteerde situatie.

Uit deze drie studies blijkt dat affectieve video op het web als context en als onderdeel van de taak de taakuitvoering beïnvloedt. De salliantie van affect, het bewustzijn van de bron ervan en de kenmerken van de taak kunnen een modererende rol spelen in hoe de affectieve responsen doorwerken op de taakuitvoering.

Deze studie steunt de notie dat niet alleen "cognitieve componenten" van de webgebaseerde leeromgeving ontworpen kunnen worden om de leereffectiviteit te verhogen, maar dat een onderwijskundig ontwerper ook "affectieve componenten" kan toepassen ten gunste van het leerproces. Echter, het doelgericht beïnvloeden van de gevoelens van anderen, vooral als dit impliciet wordt gedaan, roept onmiddellijk de ethische vraag op of het gebruik van affectieve strategieën in het onderwijs wel toelaatbaar is. In ons dagelijks leven "buiten school" zijn affectieve invloeden duidelijk aanwezig. Iedereen ervaart de trend dat video boodschappen zowel qua inhoud als qua verpakking meer emotioneel worden. Het lijkt erop dat veel is toegestaan bij het bereiken van commerciële en politieke communicatiedoelen. Maar welke middelen zijn toegestaan in het onderwijs om onderwijsdoelen te bereiken? Mogen we "instructie verkopen als tubes tandpasta"? Bij het exploiteren van het affectieve potentieel van video in een webgebaseerde leeromgeving moeten we bewust zijn van de emotionele processen op het leren. We hopen dat ons onderzoek heeft bijgedragen aan deze bewustwording en aan inzichten in deze effecten. 


\section{Dankwoord}

Eliot schreef heel treffend dat we na al ons exploreren weer terugkomen bij het begin en dan pas weten waarover het gaat. In de afgelopen jaren heb ik dit proces op allerlei gebieden doorlopen. Zo had ik meerdere uitwerkingen van dit proefschrift voor ogen, maar kwam uiteindelijk uit bij het beginidee. Het proefschrift groeide mee met de veranderende wereld van video, mijn medium dat hot was toen ik met mijn studie Toegepaste Onderwijskunde begon, dat een tijdje minder aandacht kreeg en nu weer volledig opbloeit. Ook groeide het proefschrift mee met mijn exploraties op het gebied van studie en werk, variërend van psychologie, onderwijskunde, cognitieve ergonomie, naar media psychologie en onderwijskunde. Het proefschrift doorstond het tumult van de totstandkoming en uitbreiding van ons gezin, het verlies van mijn zus Ans, de reorganisaties op de UT en mijn extracurriculaire activiteiten. Het afronden van een proefschrift is als een avontuurlijke en barre reis die zijn eind-of beginbestemming heeft bereikt. Ik wil graag iedereen bedanken die mij de afgelopen jaren hebben gesteund en mij hebben aangemoedigd. Een aantal van hen wil ik hier met naam noemen.

In de eerste plaats bedank ik natuurlijk Pløn Verhagen, mijn promotor. Volgens hem zijn we uit hetzelfde AV hout gesneden en zo voelde dat inderdaad. Een half woord was genoeg. Dat ik terugkeerde naar Twente, na een aantal jaren als onderwijskundig ontwerper actief te zijn geweest, kwam door Pløn. Je bent mijn audiovisuele "godfather" en stimuleert mij voor kwaliteit en voor "the family" te gaan. Ard Heuvelman, mijn assistent-promotor, ben ik ook heel veel dank verschuldigd. Ik kende jou al als docent en mediapsycholoog toen ik nog student(-assistent) was. Later adviseerde je mij inhoudelijk bij mijn onderzoek, we werden collega's in het vakgebied van de mediapsychologie en uiteindelijk redde je mij uit de reorganisatievijver. Sindsdien werken we met veel lol samen in de afdeling MCO van Jan van Dijk en sinds kort delen we een kamer. Van jou leerde ik om door te zetten en pragmatisch te zijn. Ook Jef Moonen wil ik bedanken. Vanaf het begin dat wij elkaar leerden kennen tijdens mijn afstuderen, maar ook later, toen ik als collega in jouw afdeling kwam werken, geloofde je in de onderzoeker in mij. Bedankt voor je vertrouwen (ook al had je mij even opgegeven) en voor je sterke, kritische opmerkingen. Gelukkig bleef je mij eraan herinneren dat er aan al het begin ook een eind moet komen. 
Verder wil ik Ingrid Heynderickx, Allison Littlejohn, Hans Beentjes en Jan van Dijk hartelijk bedanken voor het willen plaatsnemen in mijn promotiecommissie en Jules Pieters omdat hij de voorzittershamer wil hanteren. Ik wil jullie bedanken voor de gesprekken die we gehad hebben over het proefschrift, maar zeker ook daarbuiten, en ik hoop van harte in de toekomst dit contact met jullie voort te zetten.

Graag wil ik ook de collega's bedanken die hebben geholpen bij de uitvoering, verwerking en presentatie van het onderzoek. Jan Oosterhuis, die mijn eenvoudige HTML pagina's en Javascripts elk jaar weer wist om te toveren tot efficiënte, dynamisch opgebouwde pagina's, waardoor ik volledig Jan afhankelijk werd; John Anemaet, die alle online verzamelde data via slimme scripts en macro's terug wist te leiden naar een SPSS-vriendelijk bestand en Johan Jonker die hielp bij het inrichten van de experimenteerzaal, het vastleggen op foto, tot en met het ontwerpen van de omslag van dit boekje. Fantastisch heren om met jullie te mogen samenwerken. Edith Krimpen-Stoop, Bernard Veldkamp en Sebie Oosterloo hartelijk dank dat ik bij jullie langs mocht komen met mijn statistiekvragen. Geir Kaufmann wil ik bedanken voor zijn bijdrage aan de tweede studie en Allison Littlejohn en Anoush Margaryan voor het proeflezen en redigeren van artikelversies van de studies. Thanks!

Twee collega's wil ik in het bijzonder bedanken. Gerard Gervedink-Nijhuis en Ko Besuijen. Jullie zijn beiden belangrijk voor mij geweest gedurende mijn loopbaan aan de Universiteit. Jullie aanwezigheid, de gesprekken, de gezelligheid en jullie steun was onmisbaar.

Tot slot wil ik mijn familie en vrienden bedanken. Mijn moeder, vader, zussen en broer, die er altijd in geloofden dat ik er wel zou komen, wat ik ook oppakte. Mijn vrienden en studiegenoten, die altijd interesse toonden en in waren voor een gezellig etentje, borrel, filmpje of sportieve activiteit. En natuurlijk, last but not least, Eric, Lisa, Marleen en Jeroen. Ik was niet altijd de gemakkelijkste partner en moeder (druk, druk, druk), maar jullie vergaven mij steeds weer. Hartelijk dank dat jullie al mijn exploraties ondersteunen en mij laten inzien waar het werkelijk om draait. Ik hoop er weer helemaal te zijn voor jullie! 